\title{
CHARACTERISTIC NUMBERS, BORDISM THEORY AND THE NOVIKOV CONJECTURE FOR OPEN MANIFOLDS
}

\author{
JÜRGEN EICHHORN \\ Institut für Mathematik und Informatik, Universität Greifswald \\ D-17487 Greifswald, Germany \\ E-mail: eichhorn@uni-greifswald.de
}

1. Introduction. Let $\left(M^{n}, g\right)$ be closed, oriented, with triangulation $K$. Then the characteristic numbers

$$
\left\langle\omega_{1}^{i_{1}} \ldots \omega_{n}^{i_{n}},[M]\right\rangle=\omega_{i_{1} \ldots i_{n}}, \quad 1 i_{1}+\cdots+n i_{n}=n
$$

and for $n=4 k$

$$
\left\langle p_{1}^{i_{1}} \ldots p_{k}^{i_{k}},[M]\right\rangle=p_{i_{1} \ldots i_{k}}, \quad 1 i_{1}+\cdots+k i_{k}=k,
$$

are well defined. Here $\omega_{i}$ are the Stiefel-Whitney and $p_{i}$ the Pontrjagin classes.

They characterize the elements of $\Omega_{n}^{S O}$. More generally, the numbers

$$
\left\langle p_{1}^{i_{1}} \ldots p_{k}^{i_{k}} f^{*} h^{(m)},[M]\right\rangle,
$$

where $m=n-4 k, h^{(m)} \in H^{m}(X, Z), X$ a finite CW-complex and $f: M^{n} \rightarrow X$ characterize the singular bordism class of $[(M, f)] \in \Omega_{n}^{S O}(X)$ modulo torsion.

If $M^{n}$ is open (hence $K$ infinite) then $\omega_{i_{1} \ldots i_{n}}$ and $p_{i_{1} \ldots i_{k}}$ are in generally not defined. More generally, we have the following simple

Proposition 1.1. There does not exist any nontrivial number valued (vector valued) invariant which is defined for all connected oriented manifolds and which is additive w.r.t. connected sums.

There are several ways out from this situation.

1) One should admit more general ranges of definition, e.g. $K$-groups (Mishchenko, Roe et al.) and give up the condition of additivity.

2) One could impose certain restrictions, i.e. define invariants not for "all" manifolds.

3) One could work with other definitions of characteristic numbers, e.g. more analytical ones.

4) One could introduce relative characteristic numbers.

2000 Mathematics Subject Classification: 57R20, 57Q20.

The paper is in final form and no version of it will be published elsewhere. 
The fundamental criterion for establishing such numbers should be their geometrical meaning and the applicability.

We define in section 2 analytical characteristic numbers, study their invariance properties and applications. Section 3 is devoted to combinatorial characteristic numbers. In section 4, we define relative characteristic numbers and apply them to bordism theory and we study bordism theory of manifolds with nonexpanding ends and relate it with the growth of the signature. Finally, section 5 is devoted to suitable versions of the Novikov conjecture.

2. Absolute characteristic numbers for open manifolds. Let $\left(M^{4 k}, g\right)$ be closed, oriented, $g$ an arbitrary Riemannian metric, $p_{i}(M, g)$ the associated by Chern-Weil construction Pontrjagin classes, $e(M, g)$ the Euler class, $L_{k}$ the Hirzebruch polynomial. Then there are the well known equations

$$
\sigma\left(M^{4 k}\right)=\int L_{k}(M, g)=\int L_{k}\left(p_{1}(M, g), \ldots, p_{k}(M, g)\right)=\sigma(M, g)
$$

and for $\left(M^{n}, g\right)$ oriented

$$
\chi\left(M^{n}\right)=\int e(M, g)=\chi(M, g) .
$$

These equations express in particular that the r.h.s. are in fact independent of $g$ and are homotopy invariants. We proved that the space of Riemannian metrics on a manifold splits w.r.t. a canonical uniform structure into "many" components and that on a compact manifold there is only one component (cf. e.g. [7]). The independence of $g$ can be reformulated as the r.h.s. depend only on $\operatorname{comp}(g)$, since the space of Riemannian metrics on a closed manifold consists only of one component. We will extend the definitions of the 1.h.s. and the r.h.s. to certain classes of open manifolds. In some cases there even holds equality. The main questions connected with such an extension are

1) the invariance properties,

2) applications, the geometrical meaning.

It is clear that the definition of characteristic numbers via Chern-Weil construction can be extended to an open manifold if the Chern-Weil integrand is $\in L_{1}$, as a very special case if this integrand is bounded and $\left(M^{n}, g\right)$ has finite volume.

Let $\left(M^{n}, g\right)$ be an open complete manifold, $G$ a compact Lie group with Lie algebra $\mathfrak{G}, \varrho: G \rightarrow U_{N}$ or $\varrho: G \rightarrow S O_{N}$ a faithful representation, $P=P(M, G)$ a principal fibre bundle and $E=P \times{ }_{G} E_{N}$ the associated vector bundle which is endowed with a Hermitean or Riemannian metric. According to the faithfulness of $\varrho$, the connections on $P$ and $E$ are in a one-to-one correspondence, $\omega \leftrightarrow \nabla^{\omega}=\nabla$. Denote by $\mathcal{C}\left(P, B_{0}, f, p\right)=\mathcal{C}\left(E, B_{0}, f, p\right)$ the set of all connections $\omega \leftrightarrow \nabla^{\omega}=\nabla$ with bounded curvature, i.e. satisfying $\left(B_{0}\right)$ : $|R| \leq C$, where $R$ denotes the curvature and || the pointwise norm, and having finite p-action

$$
\int\left|R^{\nabla^{\omega}}\right|^{p} \operatorname{dvol}_{x}(g)<\infty .
$$


We fix $P$ and $E$ and write therefore simply $\mathcal{C}\left(B_{0}, f, p\right)$. Let $\delta>0$ and set

$$
\begin{aligned}
V_{\delta}= & \left\{\left(\nabla, \nabla^{\prime}\right) \in \mathcal{C}\left(B_{0}, f, p\right)^{2}\left|{ }^{b, 1}\right| \nabla-\left.\nabla^{\prime}\right|_{\nabla, p, 1}={ }^{b}\left|\nabla-\nabla^{\prime}\right|+{ }^{b}\left|\nabla\left(\nabla-\nabla^{\prime}\right)\right|\right. \\
& \left.+\left|\nabla-\nabla^{\prime}\right|_{p}+\left|\nabla\left(\nabla-\nabla^{\prime}\right)\right|_{p}<\delta\right\}
\end{aligned}
$$

LEMMA 2.1. $\mathfrak{B}=\left\{V_{\delta}\right\}_{\delta>0}$ is a basis for a metrizable uniform structure ${ }^{b, 1} \mathfrak{U}^{p, 1}\left(\mathcal{C}\left(B_{0}, f, p\right)\right)$.

Proof. We start with $\left(U_{2}^{\prime}\right)$ : For each $V \in \mathfrak{B}$ there exists $V^{\prime} \in \mathfrak{B}$ such that $V^{\prime} \subseteq V^{-1}$.

$$
{ }^{b, 1}\left|\nabla^{\prime}-\nabla\right|_{\nabla^{\prime}, p, 1}={ }^{b}\left|\nabla^{\prime}-\nabla\right|+{ }^{b}\left|\nabla^{\prime}\left(\nabla^{\prime}-\nabla\right)\right|+\left|\nabla^{\prime}-\nabla\right|_{p}+\left|\nabla^{\prime}\left(\nabla^{\prime}-\nabla\right)\right|_{p} .
$$

Hence we have to estimate only

$$
{ }^{b}\left|\nabla^{\prime}\left(\nabla^{\prime}-\nabla\right)\right| \leq{ }^{b}\left|\left(\nabla^{\prime}-\nabla\right)\left(\nabla^{\prime}-\nabla\right)\right|+{ }^{b}\left|\nabla\left(\nabla^{\prime}-\nabla\right)\right| \leq C^{b, 1}\left|\nabla^{\prime}-\nabla\right|^{2}+{ }^{b, 1}\left|\nabla^{\prime}-\nabla\right|
$$

and

$$
\begin{aligned}
\left|\nabla^{\prime}\left(\nabla^{\prime}-\nabla\right)\right|_{p} & \leq\left|\left(\nabla^{\prime}-\nabla\right)\left(\nabla^{\prime}-\nabla\right)\right|_{p}+\left|\nabla\left(\nabla^{\prime}-\nabla\right)\right|_{p} \\
& \leq C_{2}{ }^{b}\left|\nabla^{\prime}-\nabla\right|\left|\nabla^{\prime}-\nabla\right|_{p}+\left|\nabla\left(\nabla^{\prime}-\nabla\right)\right|_{p}
\end{aligned}
$$

i.e.

$$
{ }^{b, 1}\left|\nabla^{\prime}-\nabla\right|_{\nabla^{\prime}, p, 1} \leq P_{1}\left({ }^{b, 1}\left|\nabla^{\prime}-\nabla\right|_{\nabla, p, 1}\right),
$$

where $P_{1}$ is a polynomial without constant term. $\left(U_{2}^{\prime}\right)$ is done.

For $\left(U_{3}^{\prime}\right)$ : For each $V \in \mathfrak{B}$ there exists $W \in \mathfrak{B}$ such that $W \circ W \subseteq V$ we have to estimate in

$$
{ }^{b, 1}\left|\nabla_{1}-\nabla_{2}\right|_{\nabla_{1}, p, 1} \leq{ }^{b, 1}\left|\nabla_{1}-\nabla\right|_{\nabla_{1}, p, 1}+{ }^{b, 1}\left|\nabla-\nabla_{2}\right|_{\nabla_{1}, p, 1}
$$

only the term ${ }^{b, 1}\left|\nabla-\nabla_{2}\right|_{\nabla_{1}, p, 1}$. But

$$
\begin{aligned}
{ }^{b, 1}\left|\nabla-\nabla_{2}\right| \nabla_{1}, p, 1= & { }^{b}\left|\nabla-\nabla_{2}\right|+{ }^{b}\left|\nabla_{1}\left(\nabla-\nabla_{2}\right)\right|+\left|\nabla-\nabla_{2}\right|_{p}+\left|\nabla_{1}\left(\nabla-\nabla_{2}\right)\right|_{p} \\
\leq & { }^{b}\left|\nabla-\nabla_{2}\right|+{ }^{b}\left|\left(\nabla_{1}-\nabla\right)\left(\nabla-\nabla_{2}\right)\right|+{ }^{b}\left|\nabla\left(\nabla-\nabla_{2}\right)\right|+\left|\nabla-\nabla_{2}\right|_{p} \\
& +\left|\left(\nabla_{1}-\nabla\right)\left(\nabla-\nabla_{2}\right)\right|_{p}+\left|\nabla\left(\nabla-\nabla_{2}\right)\right|_{p} \\
\leq & { }^{b, 1}\left|\nabla-\nabla_{2}\right| \nabla, p, 1+2^{b, 1}\left|\nabla_{1}-\nabla\right|_{\nabla_{1}, p, 1} \cdot{ }^{b, 1}\left|\nabla-\nabla_{2}\right|_{\nabla, p, 1},
\end{aligned}
$$

together with $(2.3)$

$$
{ }^{b, 1}\left|\nabla_{1}-\nabla_{2}\right|_{\nabla_{1}, p, 1} \leq P_{2}\left({ }^{b, 1}\left|\nabla_{1}-\nabla\right|_{\nabla_{1}, p, 1},\left|\nabla-\nabla_{2}\right|_{\nabla, p, 1}\right),
$$

where $P_{2}$ is a polynomial without constant term. $\left(U_{3}^{\prime}\right)$ is done.

Denote by ${ }^{b, m} \Omega^{q}\left(\mathfrak{G}_{E}\right)$ or $\Omega^{q, p, r}\left(\mathfrak{G}_{E}\right)$ or ${ }^{b, m} \Omega^{q, p, r}\left(\mathfrak{G}_{E}\right)$ the completion of

$$
{ }_{m}^{b} \Omega^{q}\left(\mathfrak{G}_{E}\right)=\left\{\left.\eta \in \Omega^{q}\left(\mathfrak{G}_{E}\right)\right|^{b, m}|\eta|:=\sum_{\mu=0}^{m} \sup _{x}\left|\nabla^{\mu} \eta\right|_{x}<\infty\right\}
$$

or

$$
\begin{aligned}
\Omega_{r}^{q, p}\left(\mathfrak{G}_{E}\right): & =\left\{\left.\eta \in \Omega^{q}\left(\mathfrak{G}_{E}\right)|| \eta\right|_{p, r}:=\left(\int \sum_{i=0}^{r}\left|\nabla^{i} \eta\right|_{x}^{p} \operatorname{dvol}_{x}(g)\right)^{\frac{1}{p}}<\infty\right\} \\
{ }_{m}^{b} \Omega_{r}^{q, p}\left(\mathfrak{G}_{E}\right) & ={ }_{m}^{b} \Omega^{q}\left(\mathfrak{G}_{E}\right) \wedge \Omega_{r}^{q, p}\left(\mathfrak{G}_{E}\right)
\end{aligned}
$$

with respect to ${ }^{b, m}||$ or ||$_{p, r}$ or $\left.{ }^{b, m}\right|_{p, r}={ }^{b, m}||+||_{p, r}$, respectively. We obtain $\Omega^{q, p, d}$ etc. by replacing $\nabla \rightarrow d$.

Denote by ${ }^{b, 1} \mathcal{C}^{p, 1}\left(B_{0}, f, p\right)$ the completion w.r.t. ${ }^{b, 1} \mathfrak{U}^{p, 1}$. 
TheOREM 2.2. a) ${ }^{b, 1} \mathcal{C}^{p, 1}\left(B_{0}, f, p\right)$ is locally arcwise connected.

b) In ${ }^{b, 1} \mathcal{C}^{p, 1}\left(B_{0}, f, p\right)$ components and arc components coincide.

c) ${ }^{b, 1} \mathcal{C}^{p, 1}\left(B_{0}, f, p\right)$ has a decomposition as a topological sum

$$
{ }^{b, 1} \mathcal{C}^{p, 1}\left(B_{0}, f, p\right)=\sum_{i \in I}{ }^{b, 1} \operatorname{comp}^{p, 1}\left(\nabla_{i}\right) .
$$

d)

$$
\begin{aligned}
{ }^{b, 1} \operatorname{comp}^{p, 1}(\nabla)= & \left\{\nabla^{\prime} \in{ }^{b, 1} \mathcal{C}^{p, 1}\left(B_{0}, f, p\right)\left|{ }^{b, 1}\right| \nabla-\left.\nabla^{\prime}\right|_{\nabla, p, 1}<\infty\right\} \\
= & \nabla+\left(\text { completion of }{ }_{1}^{b} \Omega^{1}\left(\mathfrak{G}_{E}, \nabla\right) \cap \Omega_{1}^{1, p}\left(\mathfrak{G}_{E}, \nabla\right)\right. \\
& \text { w.r.t. }{ }^{b, 1}||_{\nabla, p, 1}=\nabla+{ }^{b, 1} \Omega^{1, p, 1}\left(\mathfrak{G}_{E}, \nabla\right) .
\end{aligned}
$$

Proof. The only fact to prove is a). b) and c) are consequences of a) and d) follows from $\nabla^{\prime}=\nabla+\left(\nabla^{\prime}-\nabla\right)$. Let $\varepsilon>0$ be so small that in $U_{\varepsilon}(\nabla)^{b, 1}|\cdot-\cdot|_{\nabla, p, 1}$ and the metric of ${ }^{b, 1} \mathcal{C}^{p, 1}\left(B_{0}, f, p\right)$ are equivalent. Put for $\nabla^{\prime} \in U_{\varepsilon}(\nabla),{ }^{b, 1}\left|\nabla-\nabla^{\prime}\right|_{\nabla, p, 1}<\varepsilon, \nabla_{t}:=$ $(1-t) \nabla+t \nabla^{\prime}=\nabla+t\left(\nabla^{\prime}-\nabla\right)$. If $\nabla_{\nu} \in{ }_{1}^{b} \Omega\left(\mathfrak{G}_{E}, \nabla\right) \cap \Omega_{1}^{1, p}\left(\mathfrak{G}_{E}, \nabla\right)$ and ${ }^{b, 1}\left|\nabla_{\nu}-\nabla\right|_{\nabla, p, 1} \underset{\nu \rightarrow \infty}{\longrightarrow} 0$ then $\nabla_{\nu, t}=\nabla+t\left(\nabla_{\nu}-\nabla\right) \rightarrow \nabla+t\left(\nabla^{\prime}-\nabla\right)=\nabla_{t}$, i.e. $\nabla_{t} \in{ }^{b, 1} \mathcal{C}^{p, 1}\left(B_{0}, f, p\right)$. Moreover, ${ }^{b, 1}\left|\nabla_{t_{1}}-\nabla_{t_{2}}\right|_{\nabla, p, 1}=\left|t_{1}-t_{2}\right| \cdot{ }^{b, 1}\left|\nabla^{\prime}-\nabla\right|_{\nabla, p, 1} \underset{t_{1} \rightarrow t_{2}}{\longrightarrow} 0$.

Lemma 2.3. The elements $\nabla$ of ${ }^{b, 1} \mathcal{C}^{p, 1}\left(B_{0}, f, p\right)$ satisfy $\left(B_{0}\right)$ and

$$
\int\left|R^{\nabla}\right|_{x}^{p} \operatorname{dvol}_{x}(g)<\infty .
$$

Proof. By the definition of ${ }^{b, 1} \mathcal{C}^{p, 1}$ its elements are $C^{1}$ (since they arise by uniform convergence of 0 -th and 1rst derivatives) hence $R^{\nabla}$ is defined. If $\nabla_{\nu} \rightarrow \nabla, \nabla_{\nu} \in \mathcal{C}\left(B_{0}, f, p\right)$, $\nabla=\nabla_{\nu}+\left(\nabla-\nabla_{\nu}\right)$, then, for fixed $\nu$,

$$
R^{\nabla}=R^{\nabla_{\nu}+\left(\nabla-\nabla_{\nu}\right)}=R^{\nabla_{\nu}}+d^{\nabla}\left(\nabla-\nabla_{\nu}\right)+\frac{1}{2}\left[\nabla-\nabla_{\nu}, \nabla-\nabla_{\nu}\right] .
$$

Each term of the r.h.s. of (2.4) is bounded, hence $R^{\nabla}$. Moreover $\left|R^{\nabla_{\nu}}\right| \in L_{p}, d^{\nabla}\left(\nabla-\nabla_{\nu}\right) \mid$ $\in L_{p}$ and $\left[\nabla-\nabla_{\nu}, \nabla-\nabla_{\nu}\right] \leq C \cdot{ }^{b}\left|\nabla-\nabla_{\nu}\right| \cdot\left|\nabla-\nabla_{\nu}\right| \in L_{p}$.

Now let $\omega \leftrightarrow \nabla^{\omega}=\nabla$ be given. After choice of a bundle chart with local base $s_{1}, \ldots, s_{N}:\left.U \rightarrow E\right|_{U}$ the curvature $\Omega$ will be described as $\Omega s_{i}=\sum_{j} \Omega_{i j} \otimes s_{j}$, where $\left(\Omega_{i j}\right)$ is a matrix of 2 -forms on $U, \Omega_{i j}\left(s_{k}, s_{l}\right)=\Omega_{i j, k l}=R_{i j, k l}$. An invariant polynomial $P:$ Mat $_{N} \rightarrow \mathbb{C}$ defines in the well known manner a closed graded differential form $P=P(\Omega)=P_{0}+P_{1}+\cdots$, where $P_{\nu}$ is a homogeneous polynomial, $P_{r}(\Omega)=0$ for $2 r>n$. The determinant is an example for $P$. If $\omega$ is not smooth then $P(\Omega)$ is closed in the distributional sense. Let $\sigma_{r}(\Omega)$ be the $2 r$-homogeneous part (in the sense of forms) of $\operatorname{det}\left(1+\Omega_{i j}\right)$.

LEMMA 2.4. Each invariant polynomial is a polynomial in $\sigma_{1}, \ldots, \sigma_{N}$.

Lemma 2.5. If $\omega \in{ }^{b, 1} \mathcal{C}^{p, 1}\left(B_{0}, f, p\right)$ and $r \geq 1$ then

$$
\int\left|\sigma_{r}(\Omega)\right|_{x}^{p} \operatorname{dvol}_{x}(g)<\infty .
$$

Proof. For the pointwise norm ||$_{x}$ we have $|\Omega|_{x}^{2}=\frac{1}{2} \sum_{i, j} \sum_{k<l}\left|\Omega_{i j, k l}\right|_{x}^{2}$, where $\Omega_{i j, k l}=$ $\Omega_{i j\left(e_{k}, e_{l}\right)}$ and $e_{1}, \ldots, e_{n}$ is an orthogonal base of $T_{x} M$. According to our assumption we 
have $|\Omega|_{p}^{p}=\int|\Omega|_{x}^{p}$ dvol $<\infty$ and $|\Omega|_{x} \leq b$ for all $x \in M$. The proof is done if we could estimate $\left|\sigma_{r}(\Omega)\right|_{x}$ from above by $|\Omega|_{x}$. By definition

$$
\sigma_{r}(\Omega)=\frac{1}{r !} \sum \varepsilon_{j_{1} \ldots j_{r}}^{i_{1} \ldots i_{r}} \Omega_{i_{1} j_{1}} \wedge \cdots \wedge \Omega_{i_{r} j_{r}}
$$

where summation runs over all $1 \leq i_{1}<\cdots<i_{r} \leq N$ and all permutations $\left(i_{1} \ldots i_{r}\right) \rightarrow$ $\left(j_{1} \ldots j_{r}\right)$. $\varepsilon$ denotes the sign of this permutation. We perform induction. For $r=1$ $\sigma_{1}(\Omega)=\sum \Omega_{i i}$. The inequality

$$
\left|\Omega_{i j}\right|_{x}^{2} \leq \sum_{s, t}\left|\Omega_{s t}\right|_{x}^{2}=2|\Omega|_{x}^{2}
$$

implies in particular $\left|\sigma_{1}(\Omega)\right|_{x}^{2} \leq 2 N|\Omega|_{x}^{2}$. For arbitrary forms $\varphi, \psi$ we have

$$
|\varphi \wedge \psi|_{x} \leq|\varphi|_{x} \cdot|\psi|_{x} .
$$

For forms with values in a vector bundle we have to multiply the r.h.s. of (2.8) with a constant. (2.6), (2.7), (2.8) and the induction assumption thus give

$$
\left|\sigma_{r}(\Omega)\right|_{x}^{2} \leq a \cdot|\Omega|_{x}^{2 r},
$$

together with $|\Omega|_{x}^{2} \leq b^{2}$ finally $\left|\sigma_{r}(\Omega)\right|_{x}=c \cdot|\Omega|_{x}$.

Corollary 2.6. Let $P$ be an invariant polynomial, $\omega \in{ }^{b, 1} \mathcal{C}^{p, 1}\left(B_{0}, f, p\right), r \geq 1$. Then each form $P_{r}(\Omega)$ is an element of ${ }^{b, 1} \Omega^{2, r p, 1}$.

Proof. This follows from 2.4, 2.5 and (2.8).

Denote by $H^{*, p}$ or ${ }^{b} H^{*}$ the $L_{p}$ or bounded cohomology, respectively.

Corollary 2.7. Under the assumptions of 2.6, $P$ and $\omega$ define well defined classes $\left[P_{\varrho}\left(\Omega^{\omega}\right)\right] \in H^{2 \varrho, p}(M),\left[P_{\varrho}\left(\Omega^{\omega}\right)\right] \in{ }^{b} H^{2 \varrho}(M)$.

Now the natural question arises: how does $\left[P_{\varrho}\left(\Omega^{\omega}\right)\right]$ depend on $\omega$ ? We denote $I=[0,1]$, $i_{t}: M \rightarrow I \times M$ the imbedding $i_{t}(x)=(t, x)$ and furnish $I \times M$ with the product metric $\left(\begin{array}{ll}1 & 0 \\ 0 & g\end{array}\right)$. Here we write $H^{q, p,\{d\}} \equiv H^{q, p}$ etc.

Lemma 2.8. For every $q \geq 0$ there exists a linear bounded mapping $K: \Omega^{q+1, p, d}(I \times M)$ $\rightarrow \Omega^{q, p, d}(M)$ resp. $K:{ }^{b} \Omega^{q+1, d}(I \times M) \rightarrow{ }^{b} \Omega^{q, d}(M)$ such that $d K+K d=i_{1}^{*}-i^{*}-0$.

Proof. Since $g_{I \times M}=\left(\begin{array}{ll}1 & 0 \\ 0 & g\end{array}\right)$ is an isometric imbedding and $i_{t}^{*}$ is bounded. $i_{t}^{*}$ maps into $\Omega^{q, p, d}(M)$ because $\left|d i^{*} \varphi\right|_{x}=\left|i^{*} d \varphi\right|_{x} \leq C \cdot|d \varphi|_{x}$. Denote $X_{0}=\frac{\partial}{\partial t}$ and for $\varphi \in \Omega^{q+1, p, d}(I \times M)$ $\varphi_{0}\left(X_{1}, \ldots, X_{q}\right):=\varphi\left(X_{0}, X_{1}, \ldots, X_{q}\right)$. Then $\varphi_{0} \in \Omega^{q, p, d}(I \times M),\left|\varphi_{0}\right|_{(t, x)} \leq|\varphi|_{(t, x)}$, and we define

$$
K \varphi\left(X_{1}, \ldots, X_{n}\right):=\int_{0}^{1} i_{t}^{*} \varphi_{0}\left(X_{1}, \ldots, X_{q}\right) d t .
$$

Thus $K$ is bounded too. The equation $d K+K d=i_{1}^{*}-t_{0}^{*}$ is standard. Replacing $\Omega^{q, p, d}$ by ${ }^{b} \Omega^{q, d}$ gives the same conclusions.

Lemma 2.9. Let $f, g: M \rightarrow N$ be smooth mappings, $F: I \times M \rightarrow N$ a smooth homotopy, $f^{*}, g^{*}: \Omega^{q, p, d}(N) \rightarrow \Omega^{q, p, d}(M), F^{*}: \Omega^{q, p, d}(N) \rightarrow \Omega^{q, p d}(I \times M)$ resp. $f^{*}, g^{*}:{ }^{b} \Omega^{q, d}(N) \rightarrow$ ${ }^{b} \Omega^{q, d}(M), F^{*}:{ }^{b} \Omega^{q, d}(N) \rightarrow{ }^{b} \Omega^{q, d}(I \times M)$ bounded and $\varphi \in \Omega^{q, p, d}(N)$ resp. $\varphi \in{ }^{b} \Omega^{q, d}(N)$ closed, i.e. $\varphi \in Z^{q, p}(N)$ resp. $\varphi \in{ }^{b} Z^{q}(N)$. Then we have $\left(g^{*}-f^{*}\right) \varphi \in B^{q, p}(M)$ resp. $\left(g^{*}-f^{*}\right) \varphi \in{ }^{b} B^{q}(M)$. 
Proof. We consider the case $\Omega^{q, p, d}$. According to our assumption we have $K \varphi:=K F^{*} \varphi \in$ $\Omega^{q-1, p, d}(M)$ and $\left(g^{*}-f^{*}\right) \varphi=\left(\left(F \circ t_{1}\right)^{*}-\left(F \circ i_{0}\right)^{*}\right) \varphi=\left(i_{1}^{*} F^{*}-i_{0}^{*} F^{*}\right) \varphi=\left(i_{1}^{*}-i_{0}^{*}\right) F^{*} \varphi=$ $(d K+K d) F^{*} \varphi=d K F^{*} \varphi=d K \varphi$. The case of bounded forms will be treated by the same equation.

Now we are able to prove one of our main theorems.

Theorem 2.10. Let $Q: M_{N}(C) \rightarrow C$ be an invariant polynomial, $r \geq 1, p=1$ or 2. Then each component $U$ of ${ }^{b, 1} \mathcal{C}^{p, 1}\left(B_{0}, f, p\right)$ determines uniquely a cohomology class $\left[Q_{r}\left(\Omega^{U}\right)\right] \in H^{p, 2 r}(M)$ resp. $\left[Q_{r}\left(\Omega^{U}\right)\right] \in{ }^{b} H^{2 r}(M)$.

Proof. Assume $\omega_{0}, \omega_{1} \in U$. Then, according to theorem 2.2, d) $\eta:=\omega_{1}-\omega_{0} \in$ ${ }^{b, 1} \Omega^{1, p, 1}\left(\mathfrak{G}_{E}, \omega_{0}\right)$ and $\omega_{t}=\omega_{0}+t_{\eta},-\delta<t<1+\delta$, is contained in $U$. We have to show $\left[Q_{r}\left(\Omega^{\omega_{0}}\right)\right]=\left[Q_{r}\left(\Omega^{\omega_{1}}\right)\right]$. Consider

$$
\Omega_{t}:=\Omega^{\omega}, \quad \Omega_{t}=\Omega_{0}+t d^{\omega_{0}} \eta+\frac{1}{2} t^{2}[\eta, \eta] .
$$

For all $t \in]-\delta, \delta+1\left[\right.$ we have $\int\left|\Omega_{t}\right|^{p}$ dvol $<\infty$ and $\left|\Omega_{t}\right|_{x}$ is bounded at $M$. This follows from (2.10) and the assumption $\omega_{0}, \omega_{1} \in U$. If $\left.\bar{p}:\right]-\delta, 1+\delta[\times M \rightarrow M$ denotes the projection $(t, x) \rightarrow x, P^{\prime}=\bar{p}^{*} P$ resp. $E^{\prime}=\bar{p}^{*} E$ the liftings of the bundles to ]$-\delta, 1+\delta[\times M, p$ (which covers $\bar{p}$ ) the associated mapping of the bundle spaces, then $p^{*} \omega_{0}, p^{*} \omega_{1}$ are connections for the lifted bundles.

$t p^{*} \omega_{1}+(1-t) p^{*} \omega_{0}=p^{*} \omega_{0}+t p^{*} \eta$ is again a connection $\omega^{\prime}$. According to (2.10), we have $\Omega^{\omega^{\prime}}=p^{*} \Omega_{0}+t \partial^{p^{*} \omega_{0}} p^{*} \eta+\frac{t^{2}}{2}\left[p^{*} \eta, p^{*} \eta\right] . p^{*}$ is bounded. Thus $\Omega^{\omega^{\prime}}$ is surely $p$-integrable and bounded if this holds for $d^{p^{*} \omega_{0}} p^{*} \eta$. But this follows from the equation $\left(p^{*} \omega_{0}\right)_{i j}=p^{*}\left(\omega_{0, i j}\right)$ for the connected matrix, $\eta \in{ }^{b, 1} \Omega^{1, p, d}\left(\mathfrak{G}_{E}, \omega_{0}\right)$ and from the boundedness of $p^{*} \cdot \omega^{\prime}, \Omega^{\omega^{\prime}}$ define well determined $p$-integrable resp. bounded cocycles at $]-\delta, 1+\delta\left[\times M\right.$. Let $i_{t}$ again be the mapping $x \rightarrow(t, x)$. Then $i_{0}^{*}\left(E^{\prime}, \omega^{\prime}\right)$ resp. $i_{1}^{*}\left(E^{\prime}, \omega^{\prime}\right)$ can be identified with $\left(E, \omega_{0}\right)$ resp. $\left(E, \omega_{1}\right) \cdot i_{t}, 0 \leq t \leq 1$, is a smooth bounded homotopy between $i_{0}$ and $i_{1}$. According to $2.9, i_{0}^{*} \Omega_{r}\left(\Omega^{\prime}\right)$ and $i_{1}^{*} \Omega_{r}\left(\Omega^{\prime}\right)$ are cohomologous in $H^{2 r, p}$ resp. ${ }^{b} H^{2 r}$, i.e. $Q_{r}\left(\Omega_{0}\right)$ and $Q_{r}\left(\Omega_{1}\right)$ are cohomologous in $H^{2 r, p}$ resp. ${ }^{b} H^{2 r}$.

Definition. For a component $U$ of ${ }^{b, 1} \mathcal{C}^{p, 1}\left(B_{0}, f, p\right)$ we define the $r$-th Chern class $c_{r}(P U, p)$ by

$$
c_{r}(E, U, p)=c_{r}(P, U, p):=\frac{1}{(2 \pi)^{r}}\left[\sigma_{r}\left(\Omega^{U}\right)\right] .
$$

Then we have $c_{r} \in H^{2 r, p}, c_{r} \in{ }^{b} H^{2 r}$.

REMARK 2.11. For $\omega_{0}, \omega_{1} \in{ }^{b, 1} \mathcal{C}^{p, 1}\left(B_{0}, f, p\right)$ the cocycles $\sigma_{r}\left(\Omega^{\omega_{0}} /(2 \pi i)^{r}, \sigma_{r}\left(\Omega^{\omega_{1}} /(2 \pi i)^{r}\right.\right.$ are contained in the Chern class $c_{r}(E)$ and therefore they are cohomologous, but they do not need to be cohomologous in $H^{2 r, p}$. Take for example an $\omega \in \Omega^{p, 1}\left(B_{0}, f, p\right)$ and apply a gauge transformation $g$ with $\omega-g^{*} \omega \notin b, 1 \Omega^{p, 1, d}\left(\mathfrak{G}_{E}, \omega\right)$. Then $\left|\Omega^{\omega}\right|_{x}=\left|\Omega^{g^{*} \omega}\right|_{x}$. An explicit example is given by $M=R^{2}, P=M \times U_{N}, \omega$ the canonical flat connection, the gauge transformation $g$ at the point $(x, y)$ given by $e^{i\left(x^{2}+y^{2}\right)} \cdot \mathrm{id}$, where id denotes the unit matrix. Then $\left|\omega-g^{*} \omega\right|_{(x, y)}=\left|g^{-1} d g\right|_{(x, y)}=|i(x d x-y d y) \cdot \mathrm{id}|_{(x, y)}=\left|N\left(x^{2}+y^{2}\right)\right|^{\frac{1}{2}}$ is neither bounded nor $p$-integrable. For this reason our approach above seems to be suitable for the general situation on noncompact Riemannian manifolds. 
Definition. For $\varrho: G \rightarrow O_{N}, E=P \times_{G} \mathbb{R}^{N}$ denote by $E^{c}$ or $P^{c}$ the complexification of $E$ or $P$, respectively. Any connection $\omega$ on $E$ resp. $P$ extends in a canonical manner to a connection on $E^{c}$ resp. $P^{c}$ and we have an inclusion of the components $U$ of ${ }^{b, 1} \mathcal{C}^{p, 1}\left(P, B_{0}, f, p\right)$ into the components $U^{c}$ of ${ }^{b, 1} \mathcal{C}^{p, 1}\left(P^{C}, B_{0}, f, p\right)$. Then we define the $k$-th Pontrjagin class $p_{k}(P, U, p)$ by

$$
p_{k}(P, U, p)=p_{k}(E, U, p):=(-1)^{k} c_{2 k}\left(P^{c}, U^{c}, p\right) .
$$

Let $P$ be the Pfaff polynomial for skew symmetric $2 N$-matrices, $\varrho: G \rightarrow S O_{2 N}$, $E=P \times_{G} \mathbb{R}^{2 N}$. Then for a component $U$ of ${ }^{b, 1} \mathcal{C}^{p, 1}\left(P^{C}, B_{0}, f, p\right)$ we call

$$
e(E, U, p):=\frac{1}{(2 \pi)^{N}} \operatorname{Pf}\left(\Omega^{U}\right)
$$

the Euler class of $U$. Then $e \in H^{2 N, p}(M), e \in{ }^{b} H^{2 N}(M)$.

Now come in characteristic numbers. Consider $\varrho: G \rightarrow U_{N}$, let $\operatorname{dim} M=2 k$ and $Q$ an invariant polynomial, $\omega \in{ }^{b, 1} \mathcal{C}^{p, 1}\left(P^{C}, B_{0}, f, p\right), Q\left(\Omega^{\omega}\right)=a+Q_{1}(\Omega)+\cdots+Q_{k}(\Omega)$. Then $Q_{i_{1} \ldots i_{k}}:=Q_{i_{1}} \wedge \cdots \wedge Q_{i_{k}}$ with $i_{1}+\cdots+i_{k}=k$ defines a characteristic $2 k$-form and a characteristic number $\int Q_{i_{1} \ldots i_{k}}=Q_{i_{1} \ldots i_{k}}(P, \omega)(M)$ if the latter integral exists. In particular we consider the classes $c_{i_{1} \ldots i_{k}}:=c_{i_{1}} \wedge \cdots \wedge c_{i_{k}}$ and have to ensure the existence of the corresponding integral.

LEMmA 2.12. a) If $k=1$ and $\omega \in{ }^{b, 1} \mathcal{C}^{1,1}\left(B_{0}, f, 1\right)$, then $\int_{M} c_{1}$ converges.

b) If $k>1$ and $\omega \in{ }^{b, 1} \mathcal{C}^{1,1}\left(B_{0}, f, 1\right)$ or $\omega \in{ }^{b, 1} \mathcal{C}^{2,1}\left(B_{0}, f, 2\right)$ then $\int_{M} c_{i_{1} \ldots i_{k}}$ converges.

Proof. a) is clear. We have to prove b). At each $x \in M, c_{i_{1} \ldots i_{k}}$ is a sum of monomials $a$. $\Omega_{i_{1} j_{1}} \wedge \cdots \wedge \Omega_{i_{k} j_{k}}$. According to lemma $2.5\left|\Omega_{i_{1} j_{1}} \wedge \cdots \wedge \Omega_{i_{k} j_{k}}\right|_{x} \leq D_{1} \cdot|\Omega|_{x}$ resp. $\leq D_{2} \cdot|\Omega|_{x}^{2}$ if $p=1$ resp. $p=2$.

COROLlary 2.13. Under the assumption of 2.12, for any invariant polynomial $Q$, the integral $\int_{M} Q_{i_{1} \ldots i_{k}}$ converges.

Proof. This follows from 2.4 and the proof of 2.12 .

Lemma $2.12 \mathrm{~b}$ ) is also valid in the case $\varrho: G \rightarrow O_{N}, \operatorname{dim} M=4 k$ for $p_{i_{1} \ldots i_{k}}, i_{1}+$ $\cdots+i_{k}=k, k \geq 1$, resp. in the case $\varrho: G \rightarrow S O_{2 N}, \operatorname{dim} M=N$ for the Euler form $e(E, \omega, 1, g)(2.12$ a) for $N=1,2.12$ b) for $N>1)$.

The above characteristic numbers until now are defined only for a chosen connection $\omega$. One would like that the characteristic numbers are constant on least on the components of ${ }^{b, 1} \mathcal{C}^{p, 1}\left(B_{0}, f, p\right)$. This is in fact the case for $p=1$.

THEOREM 2.14. The characteristic numbers are constant on the components of the space ${ }^{b, 1} \mathcal{C}^{1,1}\left(B_{0}, f, 1\right)$.

Proof. If $\omega, \omega^{\prime}$ are contained in the same component $U$, then according to $2.10, Q_{i_{1} \ldots i_{k}}(\omega)$ and $Q_{i_{1} \ldots i_{k}}\left(\omega^{\prime}\right)$ define the same cohomology class in $H^{2 k, 1}(M)$ resp. $H^{4 k, 1}(M)$, i.e. there exists an absolutely integrable $\varphi$ with $d \varphi=Q_{i_{1} \ldots i_{k}}(\omega)-Q_{i_{1} \ldots i_{k}}\left(\omega^{\prime}\right)$. A fundamental result of Gaffney then says $\int_{M} d \varphi=0$ for $(M, g)$ complete and $d \varphi$ itself absolutely integrable $([12])$.

Thus one gets characteristic numbers $Q_{i_{1} \ldots i_{k}}(P, U)(M)$. 
REMARK 2.15. For $\omega, \omega^{\prime} \in{ }^{b, 1} \mathcal{C}^{2,1}\left(B_{0}, f, 2\right)$ and $\operatorname{deg}\left(Q_{i_{1} \ldots i_{k}}\right) \geq 4$ the characteristic numbers $Q_{i_{1} \ldots i_{k}}(\omega)(M), Q_{i_{1} \ldots i_{k}}\left(\omega^{\prime}\right)(M)$ are defined. If $Q_{i_{1} \ldots i_{k}}(\omega), Q_{i_{1} \ldots i_{k}}\left(\omega^{\prime}\right)$ define the same cohomology class in $H^{2 k, 1}\left(M^{2 k}\right)$ resp. $H^{4 k, 1}$ resp. $H^{2 N, 1}$, then the characteristic numbers coincide.

A very special but interesting case in our considerations is the case $\operatorname{vol}(M)<\infty$. Consider ${ }^{b, 1} \mathcal{C}\left(B_{0}\right)$. It is defined by means of $\mathfrak{B}=\left\{V_{\delta}\right\}_{\delta>0}$, where $V_{\delta}=\left\{\left(\nabla, \nabla^{\prime}\right) \in\right.$ $\left.\left.\mathcal{C}\left(B_{0}\right)^{2}\right|^{b, 1}\left|\nabla-\nabla^{\prime}\right|_{\nabla}<\delta\right\}$.

THEOREM 2.16. If $\operatorname{vol}(M)<\infty$ then characteristic numbers are constant on each component of ${ }^{b, 1} \mathcal{C}\left(B_{0}\right)$.

Proof. According to 2.10 each component $U$ of ${ }^{b, 1} \mathcal{C}\left(B_{0}\right)$ determines uniquely a cohomology class $\left[Q_{i_{1} \ldots i_{k}}\right]$ in ${ }^{b} H^{2 k}\left(M^{2 k}\right)$ or ${ }^{b} H^{4 k}$ or ${ }^{b} H^{2 N}$ respectively. Taking two cocycles of this class, there exists a bounded $C^{1}$-form $\varphi$ such that there difference equals to $d \varphi$. $\varphi$, $d \varphi$ are bounded, $\operatorname{vol}(M)<\infty$, thus $\varphi, d \varphi$ are absolutely integrable and the theorem of Gaffney gives the desired result.

REMARK 2.17. $\operatorname{vol}(M)<\infty$ implies ${ }^{b, 1} \mathcal{C}\left(B_{0}\right)={ }^{b, 1} \mathcal{C}^{p, 1}\left(B_{0}, f, p\right)$. Thus the conclusion of 2.16 also holds for the components of ${ }^{b, 1} \mathcal{C}^{p, 1}\left(B_{0}, f, p\right)$.

We call the quasi isometry class of $g$ the uniform structure $U S(g)$ generated by $g$. For all metrics of $U S(g)$ the cohomology spaces $H^{*, p}\left(M^{n}, g^{\prime}\right)$ coincide. The same holds for ${ }^{b} H^{*}\left(M^{n}, g^{\prime}\right)$. This leads immediately to

THEOREM 2.18. The cohomology classes $Q_{i_{1} \ldots i_{k}}(\omega)$ resp. the characteristic numbers $Q_{i_{1} \ldots i_{k}}(\omega)(M)$ in 2.10 respectively 2.3, 2.5 are the same for all metrics $g^{\prime} \in U S(g)$.

The situation completely changes if $\omega$ itself depends on $g$. Then it is not true in general that for $g^{\prime} \in U S(g), c(\omega(g)) \sim c\left(\omega\left(g^{\prime}\right)\right)$. The case $\omega=\omega(g)$ is essentially the case $P=$ bundle of orthogonal frames of $\left(M^{n}, g\right), \nabla=$ Levi-Civita connection $\nabla^{g}$. Therefore we briefly describe the metrics which come into question and describe their admitted variation (for fixed $M$ ).

Let

$$
\begin{aligned}
\mathcal{M}\left(B_{0}, p, f\right)= & \left\{g \mid g \text { complete, satisfies }\left(B_{0}\right) \text { and } \int\left|R^{g}\right|_{x}^{p} \operatorname{dvol}_{x}(g)<\infty\right\}, \\
{ }^{b, 2}\left|g-g^{\prime}\right|_{g, p, 2}= & { }^{b, 2}\left|g-g^{\prime}\right|_{g}+\left|g-g^{\prime}\right|_{g, p, 2} \\
= & { }^{b}\left|g-g^{\prime}\right|_{g}+{ }^{b}\left|\nabla^{g}-\nabla^{g^{\prime}}\right| g+{ }^{b}\left|\nabla^{g}\left(\nabla^{g}-\nabla^{g^{\prime}}\right)\right| \\
& +\left(\int\left(\left|g-g^{\prime}\right|_{g, x}^{p}+\sum_{i=0}^{1}\left|\left(\nabla^{g}\right)^{i}\left(\nabla^{g}-\nabla^{g^{\prime}}\right)\right|_{g, x}^{p}\right) \operatorname{dvol}_{x}(g)\right)^{\frac{1}{p}}
\end{aligned}
$$

and set

$$
V_{\delta}=\left\{\left(g, g^{\prime}\right) \in \mathcal{M}\left(B_{0}, p, f\right)^{2} \mid C(n, \delta)^{-1} g \leq g^{\prime} \leq C(n, \delta) g \text { and }{ }^{b, 2}\left|g-g^{\prime}\right|_{g, p, 2}<\delta\right\} .
$$

Here $C(n, \delta)=1+\delta+\delta \sqrt{2 n(n-1)}$.

LEMMA 2.19. $\mathfrak{B}=\left\{V_{\delta}\right\}_{\delta>0}$ is a basis for a metrizable uniform structure.

Denote by ${ }^{b, 2} \mathcal{M}^{p, 2}\left(B_{0}, p, f\right)$ its completion. 
Proposition 2.20. a) ${ }^{b, 2} \mathcal{M}^{p, 2}\left(B_{0}, p, f\right)$ is locally arcwise connected.

b) In ${ }^{b, 2} \mathcal{M}^{p, 2}\left(B_{0}, p, f\right)$ coincide components with arccomponents.

c) ${ }^{b, 2} \mathcal{M}^{p, 2}\left(B_{0}, p, f\right)$ has a representation as a topological sum

$$
{ }^{b, 2} \mathcal{M}^{p, 2}\left(B_{0}, p, f\right)=\sum_{i \in I}^{b, 2} \operatorname{comp}^{p, 2}\left(g_{i}\right) .
$$

d) $\operatorname{comp}(g)=\left\{\left.g^{\prime} \in{ }^{b, 2} \mathcal{M}^{p, 2}\left(B_{0}, p, f\right)\right|^{b, 2}\left|g-g^{\prime}\right|_{g, p, 2}<\infty\right\}$.

Proposition 2.21. If $g^{\prime} \in \operatorname{comp}(g)$ then $\nabla^{g^{\prime}} \in \operatorname{comp}\left(\nabla^{g}\right)$ is the sense of theorem 2.2, $d$ ).

Hence we obtain well defined characteristic classes $C\left(\nabla^{g}\right)=C(g)$ and characteristic numbers $C \ldots\left(\nabla^{g}\right)(M)=C \ldots(g)(M)$ as above. The main important cases are the Euler form $e=E(g)$,

$$
\chi\left(M^{n}, g\right):=\int_{M} E(g)
$$

and the signature case

$$
\sigma\left(M^{n}, g\right):=\int_{M} L(g),
$$

where $L(g)$ is the Hirzebruch genus.

The following natural questions arise.

1) How does $E(g)$ depend on $g$ ?

2) What is the topological meaning of $\chi\left(M^{n}, g\right)$ ?

3) Under which conditions, $\chi\left(M^{n}, g\right)=\chi\left(M^{n}\right)$, i.e. the Gauß-Bonnet formula holds?

The same questions should be put for $\sigma\left(M^{n}, g\right), \sigma\left(M^{n}\right)$. To the first question we have a partial answer.

Proposition 2.22. If $g^{\prime} \in{ }^{b, 2} \operatorname{comp}^{1,2}(g)$ then

$$
\chi\left(M^{n}, g\right)=\chi\left(M^{n}, g^{\prime}\right)
$$

and

$$
\sigma\left(M^{n}, g\right)=\sigma\left(M^{n}, g^{\prime}\right)
$$

In the case $g^{\prime} \notin b, 2 \operatorname{comp}^{1,2}(g)$ we can't say anything. The examples in [3] for $\chi\left(M^{n}, g\right) \neq \chi\left(M^{n}, g^{\prime}\right), \sigma\left(M^{n}, g\right) \neq \sigma\left(M^{n}, g^{\prime}\right)$ are of this kind, i.e. $g^{\prime}$ does not lie in the component of $g$.

Concerning the second question, we start with a simple case in dimension two which has been discussed by Cohn-Vossen [5] and Huber [16] and has been endowed with particular short proofs by Rosenberg [22], which we present below for completeness.

TheOREM 2.23. Let $\left(M^{n}, g\right)$ be a finitely connected complete noncompact Riemannian surface with curvature $K$.

a) If $K \in L_{1}$ then $\chi(M) \geq \int_{M} K \operatorname{dvol}_{x}(g)$.

b) If $\operatorname{vol}\left(M^{2}, g\right)<\infty$ and $K \in L_{1}$ then

$$
\chi(M)=\int_{M} K \operatorname{dvol}_{x}(g)=\chi(M, g) .
$$


Proof. $M^{2}$ is diffeomorphic to a compact surface with $p$ points deleted. A neighborhood of each point is diffeomorphic to $S^{1} \times R_{+}$and the metric can be put in the form $g_{11}(\theta, t) d \theta^{2}+d t^{2}$. Set $\left.M_{k}=M \backslash \bigcup_{1}^{p} S^{1} \times\right] k, \infty[$. The Gauß-Bonnet theorem for surfaces with boundary yields $\chi\left(M_{k}\right)=\int_{M_{k}} K \operatorname{dvol}_{x}(g)+\int_{\partial M_{k}} \omega_{12}$, where $\omega_{12}$ is the connection 1-form associated to an orthonormal frame on $M \cdot \chi(M)=\chi\left(M_{k}\right)$, hence one has to show $\lim _{k \rightarrow \infty} \int_{\partial M_{k}} \omega_{12} \geq 0$ for a) and $\lim _{k \rightarrow \infty} \int_{\partial M_{k}} \omega_{12}=0$ for b). W.r.t. the orthonormal frame $\theta^{1}=\sqrt{g_{11}} d \theta$ and $\theta^{2}=d t$ the first structure equation $d \theta^{1}=\omega_{12} \cap \theta^{2}$ gives $\omega_{12}=\frac{d}{d t}\left(\sqrt{g_{11}}\right) d \theta$ and the second one gives $K \operatorname{dvol}_{x}(g)=\Omega_{12}=d \omega_{12}=\frac{d^{2}}{d t^{2}}\left(\sqrt{g_{11}}\right) d \theta d t$. $\int_{M} K \operatorname{dvol}_{x}(g)<\infty$ implies $\lim _{k \rightarrow \infty} \int_{\partial M_{k}} \frac{d^{2}}{d t^{2}} \sqrt{g_{11}} d \theta=0$ or $\lim _{k \rightarrow \infty} \int_{\partial M_{k}} \frac{d^{2}}{d t^{2}} \sqrt{g_{11}} d \theta=$ const $=C$. In the case b), $\operatorname{vol}(M, g)<\infty$, i.e. $\int_{M} \sqrt{g_{11}} d \theta d t<\infty$ which implies $\lim _{k \rightarrow \infty} \int_{\partial M_{k}} \sqrt{g_{11}} d \theta=0$, hence $\lim _{k \rightarrow \infty} \int_{\partial M_{k}} \omega_{12}=\lim _{k \rightarrow \infty} \frac{d}{d t} \int \sqrt{g_{11}} d \theta=C, C=0$. In the case a), $\int_{\partial M_{k}} \sqrt{g_{11}} d \theta \sim C \cdot k+D$ as $k \rightarrow \infty$. $C<0$ would imply $\int_{\partial M_{k}} \sqrt{g_{11}} d \theta=0$ for $k$ sufficiently large. But this is impossible for a positive integrand.

In the case of arbitrary $n$, there are many approaches to study the equation $\chi(M, g)=$ $\chi(M)$. To have $\chi(M)$ defined, one must require that each homology group over $\mathbb{R}$ is finitely generated. Sufficient for this is that $M$ has finite topological type, i.e. it has only finitely many ends $\varepsilon_{1}, \ldots, \varepsilon_{s}$, each of them collared, $U\left(\varepsilon_{i}\right) \cong \partial U_{i} \times[0, \infty[$. Then $M$ can be given a boundary $\partial M$ to get a compact manifold $\bar{M}$. The case of $n$ odd is absolutely trivial.

Proposition 2.24. Assume $\left(M^{2 k+1}, g\right)$ is of finite topological type, $g$ arbitrary. Then

$$
\chi(M)=\int_{M} E(g)=\chi(M, g) \text { if and only if } \chi(\partial M)=0 .
$$

Proof. For $n=2 k+1$, the Euler form $E(g)$ vanishes since the Pfaffian of an odd dimensional skew symmetric matrix is zero, $\int E(g)=\chi(M, g)=0$. On the other hand, $0=\chi(\bar{M} \underset{\partial M}{\cup} \bar{M})=2 \chi(\bar{M})-\chi(\partial M)=2 \chi(M)-\chi(\partial M)$.

The more interesting case are even dimensional manifolds. We recall some definitions.

For a local orthonormal frame $\theta^{1}, \ldots, \theta^{n}$ the connection 1 -forms $\omega_{i j}$ satisfy the equations

$$
d \theta^{i}=\sum_{j} \omega_{i j} \wedge \theta^{j} \text { and } \omega_{j i}=-\omega_{i j} .
$$

They are related with the curvature 2 -forms $\Omega_{i j}$ by

$$
\Omega_{i j}=d \omega_{i j}-\sum_{k} \omega_{i k} \wedge \omega_{k j}
$$

Denote by $S(M)$ the tangent sphere bundle which is a $(2 n-1)$-dimensional manifold. For a point $(x, \xi) \in S(M)$ let $\theta^{1}, \ldots, \theta^{n}$ be a frame such that $\theta^{1}$ is dual to $\xi$. We put

$$
\quad \sum_{0 \leq k<n}^{I I(g):=} c_{k} \sum_{\alpha} \operatorname{sign}(\alpha) \Omega_{\alpha(2) \alpha(3)} \wedge \cdots \wedge \Omega_{\alpha(2 k) \alpha(2 k+1)} \wedge \omega_{\alpha(2 k+2) 1} \wedge \cdots \wedge \omega_{\alpha(n) 1},
$$

where we will not specify the $c_{k}$ and $\sum_{\alpha}$ means the sum over all permutations $\alpha$ of $\{2, \ldots, n\} . I I(g)$ can be understand as pull back on an $(n-1)$-form on $M$ to $S(M)$ by means of $p r: S(M) \rightarrow M$. If $X \in T S(M)$ at $(x, \xi), X=X_{1}+X_{2}$ with $X_{1}$ tangent to 
$M$ and $X_{2}$ tangent to $S_{x}^{n-1}$ then $\Omega_{i j}(X)=\Omega_{i j}\left(X_{1}\right)$ and similarly for $\omega_{i 1}(X)$. If $M$ is compact with boundary $\partial M$ and $\varrho$ is the section of $S(M)$ over $\partial M$ given by the outward normal vector, then $\varrho^{*} \Omega_{i j}(X)=\Omega_{i j}\left(X_{1}\right)$, the same for $\omega_{i 1}$. Then, according to Chern,

$$
\chi(M)=\int_{M} E(g)+\int_{\partial M} \varrho^{*} I I(g)=\int_{M} E(g)+\int_{\partial M} I I(g) .
$$

Assume now that $\left(M^{n}, g\right)=\left(M^{2 m}, g\right)$ is even-dimensional and of finite topological type. By gradient flow of an appropriate Morse function we can introduce coordinates $\left(x_{1}, \ldots, x_{n-1}, x_{n}=r\right)$ at each end such that $0 \leq r<\infty, g_{i n}=0,1 \leq i \leq n-1, g_{n n}=1$. Let as above $M_{k}$ be characterized by $x_{n}=r \leq k$. Then

$$
\chi(M)=\chi\left(M_{k}\right)=\int_{M_{k}} E(g)+\int_{\partial M_{k}} I I(g) .
$$

At each end $\left.\varepsilon T M\right|_{\varepsilon}$ splits as $T M=W \oplus \mathbb{R}$. Suppose additionally that $W$ splits as

$$
W=W_{2} \oplus \cdots \oplus W_{r_{\varepsilon}}, \quad r_{\varepsilon} \geq 2, \quad\left[W_{i}, W_{j}\right]=0 \quad \text { if } i \neq j,
$$

and that with respect to this splitting $g$ has the form

$$
g=f_{2}^{2}(r) g_{2} \oplus \cdots \oplus f_{r_{\varepsilon}}^{2}(r) g_{r_{\varepsilon}}+d r^{2} .
$$

Then S. Rosenberg calculated in [23] the expression (2.11) at each end can could show if $f_{j}(r) \underset{r \rightarrow \infty}{\longrightarrow} 0, f_{j}^{\prime}(r) \underset{r \rightarrow \infty}{\longrightarrow} 0$, then $\int_{M_{k}} E(g) \rightarrow \int_{M} E(g)$ and $\int_{\partial M_{k}} I I(g) \rightarrow 0$. We will not repeat the really simple calculations but state Rosenberg's

THEOREM 2.25. Let $\left(M^{n}, g\right)$ be open, complete and of finite topological type. Assume that in an open neighborhood of each end $\varepsilon M$ splits as a product manifold $N_{2} \times \cdots \times$ $N_{r_{\varepsilon}} \times \mathbb{R}$ with the metric $f_{2}^{2}(r) g_{2} \oplus \cdots \oplus f_{r_{\varepsilon}}^{2}(r) g_{r_{\varepsilon}}+d r^{2}$, where $g_{j}$ is a metric on $N_{j}$. If $f_{j}(r) \underset{r \rightarrow \infty}{\longrightarrow} 0$ and $f_{j}^{\prime}(r) \underset{r \rightarrow \infty}{\longrightarrow} 0$, then $\chi(M)=\int_{M} E(g) \equiv \chi(M, g)$. In particular, any evendimensional manifold of finite topological type admits complete warped product metrics satisfying Gauß-Bonnet (setting $N_{2}=\partial M$ ).

Corollary 2.26. Assume the hypothesis of 2.25 and additionally $g \in{ }^{b, 2} \mathcal{M}^{1,2}\left(B_{0}, f, 1\right)$. If $g^{\prime} \in{ }^{b, 2} \operatorname{comp}^{1,2}(g)$ then $\chi(M)=\int_{M} E\left(g^{\prime}\right) \equiv \chi\left(M, g^{\prime}\right)$.

REMARK 2.27. We see in 2.26 a considerable improvement of 2.25 since now the admitted class of metrics is much larger.

If one gives up the integrability of the $W \mathrm{~s}$ in (2.14), i.e. the product structure of the $\varepsilon$ s then one must strengthen the conditions to the $f_{j}$. This has been done by Rosenberg too.

THEOREM 2.28. Let $\left(M^{n}, g\right)$ be open, complete and of finite topological type. Assume that in an open neighborhood of each end $\varepsilon,\left.T M\right|_{\varepsilon}=W_{2} \oplus \cdots \oplus W_{r_{\varepsilon}} \oplus \mathbb{R}$ and the metric is of the form $f_{2}^{2}(r) g_{2} \oplus \cdots \oplus f_{r_{\varepsilon}}^{2}(r) g_{r_{\varepsilon}}+d r^{2}$ with $g_{i}$ a metric on $W_{i}$. If $f_{i}(r) \underset{r \rightarrow \infty}{\longrightarrow} 0$, $f_{i}^{\prime}(r) \underset{r \rightarrow \infty}{\longrightarrow} 0$ and $f_{j} f_{i}^{-1}$ and $\left(f_{j} f_{i}^{-1}\right)^{\prime}$ are bounded for all $r, i, j$ then

$$
\chi(M)=\int E(g) .
$$


ExAmPLE. Let $M \backslash G / K$ be an arithmetic quotient of an even-dimensional split rank-one symmetric space. Then at each component $\partial M_{i}$ of $\partial M, \partial M$ is the total space of a fibration over a torus $T_{1}$ with a torus $T_{2}$ as fiber. We have $\left.T M\right|_{V \times \mathbb{R}}=W_{1} \oplus W_{2} \oplus \mathbb{R}$ for open $V \subset \partial M$ where the fibration restricted to $V$ is trivial. $W_{i}$ is the tangent space to the torus $T_{i}$. But in general the $G$-invariant metric $g$ does not respect this splitting. Donnelly has shown in [6] that each end $\varepsilon$ has the structure $N \times \mathbb{R}, N$ at most two-step nilpotent. The Lie algebra $\mathfrak{n}$ of $N$ splits as a sum $\mathfrak{n}=V_{2} \oplus V_{3}$ of root spaces, $V_{3}=Z(\mathfrak{n})$, and the invariant metric at the identity of $N$ has the form

$$
e^{-2 r} g_{2}+e^{-4 r} g_{3}+d r^{2}
$$

where $g_{2}$ is a metric on $V_{2}, g_{3}$ a metric on $V_{3}$. [n, $\left.\mathfrak{n}\right] \subset Z(\mathfrak{n})$ and the $G$-invariant distribution $V_{2}$ is not integrable. Hence theorem 2.25 is not applicable in general. In the hyperbolic case $G / K=S O(n, 1) / S O(n)$, one has $V_{2}=\mathfrak{n}$, which yields Gauß-Bonnet.

Corollary 2.29. Assume that the hypotheses of theorem 2.28 hold and additionally $g \in{ }^{b, 2} \mathcal{M}^{1,2}\left(B_{0}, f, 1\right)$. If $g^{\prime} \in{ }^{b, 2} \operatorname{comp}^{1,2}(g)$ then $\chi(M)=\int E\left(g^{\prime}\right) \equiv \chi\left(M, g^{\prime}\right)$.

There is another Gauß-Bonnet case which does not fall under 2.2-2.29.

Proposition 2.30. Let $\left(M^{2 m}, g\right)$ be open, complete, of finite topological type and the metric at $\infty$ constant with respect to $r$, i.e. there exists an $r_{0} \geq 0$ such that $g\left(r_{1}, x\right)=$ $g\left(r_{2}, x\right)$ for all $x \in \partial M$ and $r_{1}, r_{2}>r_{0}$. Then

$$
\chi(M)=\int_{M} E(g) \equiv \chi(M, g) .
$$

Proof. Let $k>r_{0}+\delta$. Then $M_{k} \cup M_{k}$ yields a smooth closed manifold. Hence

$$
\begin{aligned}
\chi\left(M_{k} \cup M_{k}\right) & =\int_{M_{k} \cup M_{k}} E\left(g_{M_{k} \cup M_{k}}\right)=2 \int_{M_{k}} E\left(g_{M_{k}}\right), \\
\chi\left(M_{k} \cup M_{k}\right) & =2 \chi\left(M_{k}\right)-\chi\left(\partial M_{k}\right)=2 \chi(M) \\
\chi(M) & =\int_{M_{k}} E\left(g_{M_{k}}\right) .
\end{aligned}
$$

Forming $\lim _{k \rightarrow \infty}$ in (2.17) gives the desired result.

A special case of 2.28 would be a metric cylinder at infinity, $\left.g\right|_{U(\infty)}=g_{\partial M} \otimes+d r^{2}$. This is simultaneously a warped product with warping function $f(r)=1$. $f(r)=1$ does not satisfy $f(r) \underset{r \rightarrow \infty}{\longrightarrow} 0,2.25$ is not applicable. Clearly, such an $\left(M^{2 m}, g\right)$ satisfies $\left(B_{0}\right)$ but either $\int_{U(\infty)}|R|^{p} \operatorname{dvol}_{x}(g)=0$ or $\int_{U(\infty)}|R|^{p} \operatorname{dvol}_{x}(g)=\infty$, similarly either $\int_{U(\infty)}|E(g)| \operatorname{dvol}_{x}(g)=0$ or $\int_{U(\infty)}|E(g)| \operatorname{dvol}_{x}(g)=\infty$. In the second case $\int E(g)$ exists but $|E(g)| \notin L_{p}, p \geq 1$.

Another class of examples which yields very useful insights are surfaces of revolution. We state from [23] without proof

Proposition 2.31. Let $f:] 0, \infty\left[\rightarrow \mathbb{R}\right.$ be smooth, $f(0)=f^{\prime}(0)=0$ and $\left(M^{2}=\{z=\right.$ $\left.f\left(x^{2}+y^{2}\right)\right\}$, induced metric from $\left.\mathbb{R}^{3}\right)$ be the associated surface of revolution. Then

$$
\chi(M)=\frac{1}{2 \pi} \int_{M} K \operatorname{dvol}_{x}(g)=\chi(M, g)
$$


if and only if

$$
r^{\frac{1}{2}} f^{\prime}(r) \underset{r \rightarrow \infty}{\longrightarrow} \pm \infty
$$

Hence, if $f$ is for all $r>0$ strongly convex or concave, (2.18) holds. In both cases $M$ has for $r>0$ positive curvature and infinite volume. On the other hand, we have 2.15 in the case of $2.23(\mathrm{~b})$ in the finite volume case, i.e. one can have $\chi(M)=\chi(M, g)$ as in the finite volume case. For this reason we should find additional conditions which assure in the finite volume case or the infinite volume case, respectively, that

1) $\chi(M, g)$ is a (proper) homotopy invariant,

2) $\chi(M, g)=\chi(M)$ if $M$ has finite topological type.

We start with $\operatorname{vol}\left(M^{n}, g\right)<\infty$ and $|K| \leq 1$ where the latter (after rescaling) is equivalent to $\left(B_{0}\right)$. Then

$$
\chi(M, g)=\int_{M} E(g)
$$

is well defined and for $g^{\prime} \in{ }^{b, 2} \operatorname{comp}^{1,2}(g)$

$$
\chi(M, g)=\chi\left(M, g^{\prime}\right) .
$$

Lemma 2.32. Let $\left(M^{n}, g\right)$ be complete, $\operatorname{vol}(M, g)<\infty$ and $|K| \leq 1$. Then $M^{n}$ admits an exhaustion by compact manifolds with smooth boundary, $M_{1}^{n} \subset M_{2}^{n} \subset \cdots, \bigcup_{k} M_{k}^{n}=M$, such that $\operatorname{vol}\left(\partial M_{k}^{n}\right) \rightarrow 0$ and for which the second fundamental forms $I I\left(\partial M_{k}^{n}\right)$ are uniformly bounded.

Proof. This is just a corollary of theorem 2.33 below.

If we take such an exhaustion as just described then

$$
\chi\left(M_{k}^{n}\right)=\chi\left(M_{k}^{n}, g\right)+\int_{\partial M_{k}^{n}} I I\left(\partial M_{k}^{n}\right) .
$$

$\int_{\partial M_{k}^{n}} I I(\partial M, g) \underset{k \rightarrow \infty}{\longrightarrow} 0, \chi\left(M_{k}^{n}\right) \in \mathbb{Z}$, hence for $k$ sufficiently large $\chi\left(M_{k}^{n}, g\right) \in \mathbb{Z}$, but we are far from a certain convergence of $\left(\chi\left(M_{k}^{n}, g\right)\right)_{k}$ and don't know anything about the topological properties of such a limit if it exists. To obtain more insight and definite results we follow [3] and consider the following additional hypothesis.

For some neighborhood $U(\infty) \subset M$, some profinite or normal covering space $\tilde{U}(\infty)$ has the injectivity radius at least (say) 1 for the pull back metric,

$$
\left.r_{\text {inj }}\left(\tilde{U}_{\infty}\right)\right) \geq 1 \text {. }
$$

Together with $|K| \leq 1$ on $\tilde{U}(\infty)$ we write geo $_{\infty}(M) \leq 1$. If $U=M$ then we denote $\operatorname{geo}(\tilde{M}) \leq 1$. In any case we assume in this hypothesis that $\tilde{U}$ or $\tilde{M}$ are profinite or normal coverings.

Here $\tilde{M} \rightarrow M$ is profinite if there exists a decreasing sequence $\left\{\Gamma_{j}\right\}_{j}$ of subgroups of finite index, $\Gamma_{j} \subset \pi_{1}(M)$, such that $\bigcap \Gamma_{j}=\pi_{1}(\tilde{M})$.

The key for everything is the following very general theorem which assures the existence of sufficiently "smooth" exhaustions and which yields 2.32 in the case of $\operatorname{vol}(M, g)<\infty$. 
ThEOREM 2.33 (Neighborhoods of bounded geometry). Let $\left(M^{n}, g\right)$ be complete, $X \subset M^{n}$ a closed subset and $0<r \leq 1$. Then there is a submanifold $U^{n}$ with smooth boundary $\partial U^{n}$ such that for some constant $c(n)$ depending only on $n$
a) $X \subset U \subset T_{r}(x)=r$-tubular neighborhood of $X$,
b) $\operatorname{vol}(\partial U) \leq c(n) \cdot \operatorname{vol}\left(T_{r}(X) \backslash X\right) \cdot r^{-1}$,
c) $|I I(\partial U)| \leq c(n) \cdot r^{-1}$.

We refer to [4] for the proof.

Now we will discuss $\chi(M, g)$ in the profinite or normal case, $\operatorname{geo}(\tilde{M}) \leq 1$. Here we follow [3]. Put for $j: A_{1} \subset A_{2}$ and real coefficients $\beta^{i}\left(A_{1}, A_{2}\right)=\operatorname{dim}\left\{j^{*}\left(H^{i}\left(A_{2}\right)\right)\right.$ $\left.\subset H^{i}\left(A_{1}\right)\right\}$ and $\beta^{i}(A)=\operatorname{dim}\left\{j^{*}\left(H^{i}(A, \partial A)\right) \subset H^{i}(A)\right\} . b^{i}$ shall denote the usual Betti number. Then for $A_{1} \subset A_{2} \subset A_{3} \subset A_{4}$ and $A \subset Y$ a finite closed and $f: Y \rightarrow Z$, $g: Z \rightarrow Y$ simplicial, determining a homotopy equivalence,

$$
\beta^{i}\left(A_{1}\right) \subseteq \beta^{i}\left(A_{2}\right) \leq \beta^{i}\left(A_{2}, A_{4}\right) \leq \beta^{i}\left(A_{3}, A_{4}\right)
$$

and

$$
\beta^{i}(A, Y) \leq \beta^{i}(f(A), Z) \leq \beta^{i}(g \circ f(A), Y) .
$$

Put for $p: \tilde{Y}^{n} \rightarrow Y^{n}$ profinite with ind $\left(\Gamma_{j}\right)=d_{j}$ and corresponding covering spaces $p_{j}: \tilde{Y}_{j}^{n} \rightarrow Y^{n}$

$$
\sup \tilde{\chi}\left(Y^{n}\right):=\varlimsup_{A \rightarrow \infty} \varlimsup_{j \rightarrow \infty} \sum_{i=1}^{n}(-1)^{i} \frac{1}{d_{j}} \beta^{i}\left(P_{j}^{-1}(A), \tilde{Y}_{j}^{n}\right) \leq \infty
$$

and define inf $\tilde{\chi}\left(Y^{n}\right)$ similarly. $A \rightarrow \infty$ is defined by partial ordering of finite subcomplexes induced by inclusion. Using (2.24) and a diagonal argument, there are subsequences $S=\tilde{Y}_{j(e)}^{n}$ s.t.

$$
\begin{aligned}
\infty \geq \tilde{\beta}^{i}\left(Y^{n}, S\right) & :=\lim _{A \rightarrow \infty} \varlimsup_{e \rightarrow \infty} \frac{1}{d_{j(e)}} \beta^{i}\left(P_{j(e)}^{-1}(A), \tilde{Y}_{j(e)}^{n}\right) \\
& =\lim _{A \rightarrow \infty} \varliminf_{e \rightarrow \infty} \frac{1}{d_{j(e)}} \beta^{i}\left(P_{j(e)}^{-1}(A), \tilde{Y}_{j(e)}^{n}\right)
\end{aligned}
$$

exists. From $(2.25)$ we infer immediately that $\tilde{\beta}^{i}\left(Y^{n}, S\right)$ is a homotopy invariant. Suppose $\tilde{\beta}^{i}\left(Y^{n}, S\right)<\infty, i=0, \ldots, n$ and $\sup \tilde{\chi}\left(Y^{n}\right)=\inf \tilde{\chi}\left(Y^{n}\right)$, then the latter number is also a homotopy invariant.

TheOREM 2.34. Suppose $\left(M^{n}, g\right)$ complete, $\operatorname{vol}\left(M^{n}, g\right)<\infty, \tilde{M}$ either profinite or normal and $\operatorname{geo}(\tilde{M}) \leq 1$.

a) Then $\chi\left(M^{n}, g\right)$ is a proper homotopy invariant,

b) in the case $\tilde{M}$ profinite

$$
\chi(M, g)=\sup \tilde{\chi}(M)=\inf \tilde{\chi}(M),
$$

c) if additionally $M$ has finite topological type,

$$
\chi(M, g)=\chi(M) .
$$


Proof. Assume $\tilde{M} \rightarrow M$ profinite, let $M_{1} \subset M_{2} \subset \cdots, \bigcup_{k} M_{k}=M$ be an exhaustion of $M$ by compact submanifolds with boundary and denote $M_{k}-R=\left\{x \in M_{k} \mid \operatorname{dist}\left(x, \partial M_{k}\right)=R\right\}$. For $j$ sufficiently large, theorem 2.33 is applicable and we apply it to $p_{j}^{-1}\left(M_{k}\right)-1, p_{j}^{-1}\left(M_{k}\right)$ with $\varepsilon=\frac{1}{2}$. This yields submanifolds $A_{j k} \subset p_{j}^{-1}\left(M_{k}\right) \subset B_{j k}$. Given $\varepsilon>0$ arbitrary, there exist $k_{0}, N(k)$ such that for $k>k_{0}, j>N(k)$

$$
\begin{aligned}
\left|\chi\left(M^{n}, g\right)-\frac{1}{d_{j}} \chi\left(B_{j k}\right)\right| \leq & \left|\chi\left(M^{n}, g\right)-\frac{1}{d_{j}} \int_{B_{j k}} E(g)\right| \\
& +\left|\frac{1}{d_{j}} \int_{B_{j k}} E(g)-\frac{1}{d_{j}} \chi\left(B_{j k}\right)\right|<\varepsilon .
\end{aligned}
$$

We see this immediately from (2.12) and (2.22), (2.23): $\chi\left(M^{n}, g\right)=\chi\left(M_{k}^{n}, g\right)+$ $\chi\left(M^{n} \backslash M_{k}^{n}, g\right)$, here $\left|\chi\left(M^{n} \backslash M_{k}^{n}, g\right)\right|$ becomes arbitrarily small for $k$ sufficiently large.

$$
\begin{aligned}
\left|\chi\left(M^{n}, g\right)-\frac{1}{d_{j}} \int_{B_{j k}} E(g)\right| \leq & \left|\chi\left(M^{n}, g\right)-\chi\left(M_{k}^{n}, g\right)\right|+\left|\chi\left(M_{k}^{n}, g\right)-\frac{1}{d_{j}} \int_{B_{j k}} E(g)\right| \\
\left|\chi\left(M_{k}^{n}, g\right)-\frac{1}{d_{j}} \int_{B_{j k}} E(g)\right| \leq & \left|\chi\left(M_{k}^{n}, g\right)-\frac{1}{d_{j}} \int_{P_{j}^{-1}\left(M_{k}^{n}\right)} E(g)\right| \\
& +\left|\frac{1}{d_{j}} \int_{P_{j}^{-1}\left(M_{k}^{n}\right)} E(g)-\frac{1}{d_{j}} \int_{B_{j k}} E(g)\right| \\
= & \left|\frac{1}{d_{j}} \int_{B_{j k} \backslash P_{j}^{-1}\left(M_{k}^{n}\right)} E(g)\right|,
\end{aligned}
$$

but this becomes arbitrarily small for $j$ and $k$ sufficiently large. Finally

$$
\left|\frac{1}{d_{j}} \int_{B_{j k}} E(g)-\frac{1}{d_{j}} \chi\left(B_{j k}\right)\right|=\left|\frac{1}{d_{j}} \int_{\partial B_{j k}} I I\left(\partial B_{j k}\right)\right| \underset{j, k \rightarrow \infty}{\longrightarrow} 0
$$

according to (2.22). (2.28) is proven.

We obtain from $(2.24)$

$$
\beta^{i}\left(A_{j k}\right) \leq \beta^{i}\left(p_{j}^{-1}\left(M_{k}\right)\right) \leq \beta^{i}\left(p_{j}^{-1}\left(M_{k}\right), \tilde{M}_{j}\right) \leq b^{i}\left(B_{j k}\right)
$$

and from the exact cohomology sequence of the pair $\left(B_{j k}, \overline{B_{j k} \backslash A_{j k}}\right)$ together with the excision property

$$
\begin{gathered}
\left|\beta^{i}\left(A_{j k}\right)-b^{i}\left(B_{j k}\right)\right| \leq b^{i-1}\left(\overline{B_{j k} \backslash A_{j k}}\right)+b^{i}\left(\overline{B_{j k} \backslash A_{j k}}\right): \\
\cdots \rightarrow H^{i-1}\left(\overline{B_{j k} \backslash A_{j k}}\right) \rightarrow H^{i}\left(B_{j k}, \overline{B_{j k} \backslash A_{j k}}\right) \rightarrow H^{i}\left(B_{j k}\right) \rightarrow H^{i}\left(\overline{B_{j k} \backslash A_{j k}}\right) \rightarrow \cdots \\
\cong H^{i}\left(A_{j k}, \partial A_{j k}\right)
\end{gathered}
$$

The manifold $\overline{B_{j k} \backslash A_{j k}}$ satisfies $\left(B_{0}\right),(I)$ for $j>N(k)$ and for $k$ sufficiently large,

$$
\operatorname{vol}\left(\overline{B_{j k} \backslash A_{j k}}\right) \leq d_{j} \varepsilon .
$$

According to a theorem of Gromov,

$$
\sum_{i} \beta^{i}\left(\overline{B_{j k} \backslash A_{j k}}\right) \leq c(n) \cdot \operatorname{vol}\left(\overline{B_{j k} \backslash A_{j k}}\right) .
$$


We infer from $(2.29)-(2.32)$ that we can replace in $(2.28) \chi\left(B_{j k}\right)$ by $\chi\left(p_{j}^{-1} M_{k}, \tilde{M}_{j}\right)$, hence

$$
\left|\chi\left(M^{n}, g\right)-\frac{1}{d_{j}} \chi\left(p_{k}^{-1}\left(M_{k}\right), \tilde{M}_{j}\right)\right|
$$

becomes arbitrarily small, any proper homotopy equivalence preserves a subsequence of $\left(\frac{1}{d_{j}} \chi\left(p_{j}^{-1}\left(M_{k}\right), \tilde{M}_{j}\right)_{j, k}, \chi\left(M^{n}, g\right)\right.$ is a proper homotopy invariant. By the same argument we conclude in the profinite case assertion b). If $M$ has finite topological type then for $k$ sufficiently large $\beta^{i}\left(p_{j}^{-1}\left(M_{k}\right), \tilde{M}_{j}\right)=\beta^{i}\left(\tilde{M}_{j}\right)$ and

$$
\chi\left(p_{j}^{-1}\left(M_{k}\right), \tilde{M}_{j}\right)=\chi\left(\tilde{M}_{j}\right) \cdot \frac{1}{d_{j}} \chi\left(\tilde{M}_{j}\right)=\chi\left(M_{j}\right)=\chi(M)
$$

yields assertion c).

The case of a normal covering $\tilde{M} \rightarrow M$ will be discussed in theorem 2.38.

The second characteristic number of particular importance is given by $\sigma(M, g)=$ $\int_{M} L(M, g)$, where $L(M, g)$ is the Hirzebruch genus. For closed $M$ it is the topological index of the signature operator, i.e. it coincides with the topological signature. For simple open manifolds this equality does not longer hold in general, as we see in section 5 . Nevertheless, we could ask for $\sigma(M, g)$ the same questions as for $\chi(M, g)$, the question for the invariance properties and the topological significance of $\sigma(M, g)$. Concerning the invariance, a first answer is given by proposition 2.22 .

But we consider also other derivations of $g$. A key role plays again the formula for the compact case with boundary, $\partial M=N$,

$$
\sigma(M, g)+\eta(N, g)+\int_{N} I I_{\sigma}(N, g)=\sigma(M),
$$

where $I I_{\sigma}(N, g)$ essentially involves the second fundamental form and $\eta(N, g)$ is the eta invariant. If $M^{n}$ is open and $M_{1} \subset M_{2} \subset \cdots, \bigcup_{k} M_{k}=M$, an appropriate exhaustion such that $\int I I_{\sigma}\left(\partial M_{k}\right) \rightarrow 0$ and $\eta\left(\partial M_{k}\right) \rightarrow 0$ then we would have in fact $\sigma\left(M_{k}, g\right)$ $\rightarrow \sigma(M)$. Hence we should ask for conditions which assure $\eta\left(\partial M_{k}\right) \rightarrow 0$. There is a clear (and for our case complete) answer.

THEOREM 2.35. Let $\left(N^{4 l-1}, g\right)$ be compact satisfying geo $(N) \leq 1$. Then there is a constant $c=c(4 l-1)$ such that

$$
\left|\eta\left(N^{4 l-1}\right)\right| \leq c(4 l-1) \cdot \operatorname{vol}\left(N^{4 l-1}, g\right) .
$$

We refer to [3], [7] for the proof.

Now we define $\sup \tilde{\sigma}(M), \inf \tilde{\sigma}(M)$ quite analogous to the Euler characteristic as follows. Let $M^{4 l}$ be complete, $\tilde{M}^{4 l} \rightarrow M$ profinite and $M_{k}^{4 l} \subset M^{4 l}$ a compact submanifold with boundary. Put

$$
\begin{aligned}
\sup \tilde{\sigma}\left(M_{k}\right) & :=\lim _{j} \sup \frac{1}{d_{j}} \sigma\left(P_{j}^{-1}\left(M_{k}\right)\right), \\
\sup \tilde{\sigma}(M) & \left.:=\lim _{M_{k}} \sup \sup \tilde{\sigma}\left(M_{k}\right)\right)
\end{aligned}
$$

and similarly $\inf \tilde{\sigma}\left(M_{k}\right)$, inf $\tilde{\sigma}(M)$. Here as always $\sigma\left(M_{k}\right)$ is defined as the signature of the cup product pairing on $j^{*} H^{2 l}\left(M_{k}^{4 l}, \partial M_{k}^{4 l}\right) \subset H^{2 l}\left(M_{k}^{4 l}\right)$. 
TheOREM 2.36. Let $\left(M^{4 l}, g\right)$ be complete, $\operatorname{vol}(M, g)<\infty$ and suppose $\tilde{M}$ either profinite or normal and $\operatorname{geo}(\tilde{M}) \leq 1$. Then we have

a) Assume $\tilde{M}$ normal. Then $\sigma(M, g)$ is a proper homotopy invariant of $M$.

b) In the case $\tilde{M} \rightarrow M$ profinite, for any exhaustion $M_{1} \subset M_{2} \subset \cdots, \bigcup_{k} M_{k}=M$, by compact manifolds,

$$
\sigma(M, g)=\sup \tilde{\sigma}(M)=\inf \tilde{\sigma}(M)
$$

c) If, additionally, $M$ has finite topological type,

$$
\sigma(M, g)=\lim _{j \rightarrow \infty} \frac{1}{d_{j}} \sigma\left(\tilde{M}_{j}\right)
$$

Proof. In the normal case $\tilde{M} \rightarrow M$ below a) follows from theorem 2.38. The proof of $\mathrm{b}$ ) is quite analogous to that of theorem $2.34 \mathrm{~b}$ ), using a chopping of $M$ according to theorem 2.33, (2.32) and theorem 2.35. c) then follows from b) and the fact that for sufficiently large $k, \frac{1}{d_{j}} \sigma\left(p_{j}^{-1}\left(M_{k}\right)\right)=\frac{1}{d_{j}} \sigma\left(\tilde{M}_{j}\right)$.

We now turn to the normal case $\tilde{M} \rightarrow M$, being even more explicit than in the profinite case. The first key here is the extension of Atiyah's $L_{2}$-index theorem for normal coverings $\tilde{M} \rightarrow M$ of closed $M$ to normal coverings $\tilde{M} \rightarrow M, M=\tilde{M} / \Gamma, r_{\text {inj }}(\tilde{M}) \geq 1$, $\left(M^{n}, g\right)$ complete, $\operatorname{vol}\left(M^{n}, g\right)<\infty,|K| \leq 1$. We denote by $\mathcal{H}^{q, 2}(\tilde{M})$ the space of $L_{2^{-}}$ harmonic $q$-forms, by $P_{\mathcal{H}^{q, 2}}: L_{2}\left(\Lambda^{q} T^{*} A\right)=\Omega^{q, 2} \rightarrow \mathcal{H}^{q, 2}$ the orthogonal projection. $P_{\mathcal{H}}$ has Schwartz kernel $\tilde{h}^{q}(x, y)$ which is a symmetric $C^{\infty}$ double form whose pointwise norm satisfies

$$
\left|h^{q}(x, y)\right| \leq c(n) .
$$

(2.34) comes from geo $(\tilde{M}) \leq 1$ and the elliptic estimate for the Laplacian. $\tilde{h}^{q}(x, y)$ is invariant under the isomtries $\Gamma$, hence the pointwise $\operatorname{trace} \operatorname{tr} \tilde{h}^{q}(x, x)$ can be understood as function on $M$ and we put as usual

$$
\tilde{b}^{q, 2}(M):=\operatorname{tr}_{\Gamma} P_{\mathcal{H}^{q, 2}(\tilde{M})}=\int_{M} \operatorname{tr} \tilde{h}^{q}(x, x) \operatorname{dvol}_{x}(g)<\infty .
$$

$\tilde{b}^{q, 2}(M)$ is just the von Neumann dimension $\operatorname{dim}_{\Gamma} \bar{H}^{q, 2}(\tilde{M})$ of the $\Gamma$-module $\bar{H}^{q, 2}(\tilde{M})$. Now we define the $L_{2}$-Euler characteristic and $L_{2}$-signature by

$$
\tilde{\chi}_{(2)}(M):=\sum_{q=0}^{n}(-1)^{q} \tilde{b}^{q, 2}(M) \quad \text { and } \quad \tilde{\sigma}_{(2)}(M):=\operatorname{tr}_{\Gamma}\left(* P_{\mathcal{H}^{2 k, 2}\left(\tilde{M}^{4 k}\right)}\right) .
$$

Now we state the $L_{2}$-index theorem for open manifolds with finite volume and bounded curvature.

Theorem 2.37. Suppose $(M, g)$ complete with $\operatorname{vol}\left(M^{n}, g\right)<\infty,|K| \leq 1$ and $\tilde{M} \rightarrow M$ normal with $\operatorname{geo}(\tilde{M}) \leq 1$. Then

$$
\chi(M, g)=\tilde{\chi}_{(2)}(M)
$$

and

$$
\sigma(M, g)=\tilde{\sigma}_{(2)}(M)
$$


We refer to [2], [7] for the proof.

We recall the existence of good chopping sequences $M_{1} \subset M_{2} \subset \cdots, \bigcup_{1}^{\infty} M_{k}=M$, $\operatorname{vol}\left(\partial M_{k}\right) \rightarrow 0,\left|I I\left(\partial M_{k}\right)\right| \leq c,\left|\tilde{h}_{k}^{q}(x, y) \leq c(n)\right|$, where $\tilde{h}_{k}^{q}$ denotes the kernel corresponding to projection on the harmonic $q$-forms for $p^{-1}\left(M_{k}\right) \subset \tilde{M}$. Then we obtain

$$
\lim _{k \rightarrow \infty} \tilde{b}^{q, 2}\left(\partial M_{k}\right)=0
$$

and

$$
\lim _{k \rightarrow \infty} \tilde{b}^{q, 2}\left(M \backslash M_{k}, \partial\left(M \backslash M_{k}\right)\right)=0 .
$$

Define $\tilde{\beta}^{q, 2}(B)$ by

$$
\tilde{\beta}^{q, 2}(B):=\operatorname{dim}_{\Gamma} \operatorname{im}\left(H^{q, 2}\left(p^{-1}(B), p^{-1}(\partial B)\right) \subset \bar{H}^{q, 2}\left(p^{-1}(B)\right)\right)
$$

and for $A \subset B$

$$
\tilde{\beta}^{q, 2}(A, B):=\operatorname{dim}_{\Gamma} \operatorname{im}\left(\bar{H}^{q, 2}\left(p^{-1}(B)\right) \subset \bar{H}^{q, 2}\left(p^{-1}(A)\right)\right) .
$$

It follows from the properties of $\operatorname{dim}_{\Gamma}$ that

$$
\tilde{\beta}^{q, 2}(A) \leq \tilde{\beta}^{q, 2}(B)
$$

and

$$
\tilde{\beta}^{q, 2}(A) \leq \tilde{\beta}^{q, 2}(A, B) \leq \tilde{b}^{2, q}(A) .
$$

We remark that (2.41) and (2.42) are the reformulation of $(2.24),(2.29)$ in the language of $\operatorname{dim}_{\Gamma}$. We established in theorem 2.37 the equations $\chi(M, g)=\tilde{\chi}_{(2)}(M), \sigma(M, g)=\tilde{\sigma}_{2}(M)$. Now we discuss the invariance properties of the right hand sides. This is the content of

TheOREM 2.38. Let $\left(M^{n}, g\right)$ be complete, $|K| \leq 1, \operatorname{vol}(M, g)<\infty$ and assume for some normal covering $\operatorname{geo}(\tilde{M}) \leq 1$.

a) If $M_{1} \subset M_{2} \subset \cdots, \cup M_{k}=M$ is an exhaustion then

$$
\lim _{k \rightarrow \infty} \tilde{\beta}^{q, 2}\left(M_{k}\right)=\lim _{k \rightarrow \infty} \lim _{l \rightarrow \infty} \tilde{\beta}^{q, 2}\left(M_{k}, M_{l}\right)=\tilde{b}^{q, 2}(M) .
$$

This implies the homotopy invariance of the $\tilde{b}^{q, 2}(M)$.

b) $\chi(M, g)$ resp. $\sigma(M, g)$ is a homotopy invariant resp. proper homotopy invariant of $M$.

c) If $M$ has the topological type of some $M_{k} \subset M$, then

$$
\tilde{b}^{q, 2}\left(M_{k}\right)=\tilde{b}^{q, 2}(M)
$$

and

$$
\chi(M, g)=\chi\left(M_{k}\right) .
$$

Proof. b) follows immediately from theorem 2.37 and a). For c) suppose that $M$ has finite topological type. Then there exists an exhaustion $M_{1} \subset M_{2} \subset \cdots$ s.t. each inclusion $M_{k} \rightarrow M$ is a homotopy equivalence. This implies

$$
\tilde{\beta}^{q, 2}\left(M_{k}, M_{k}\right)=\tilde{b}^{q, 2}\left(M_{k}\right)
$$

and we obtain (2.44) from (2.43) and moreover $\chi(M, g)=\chi\left(M_{k}\right)$. Hence there remains to show a). For this we must refer to [2].

We apply these results on characteristic numbers to 4-manifolds. 
Let $\left(M^{4}, g\right)$ be open, complete and oriented, $*: \Lambda^{2} M \rightarrow \Lambda^{2} M$ the Hodge operator, $*^{2}=1, \Lambda^{2}=\Lambda_{+}^{2} \oplus \Lambda_{-}^{2}$. The special orthogonal group acts on the space of algebraic curvature tensors $\mathcal{C}_{b}^{2}$ (cf. [21]). Let $\mathcal{C}_{b}^{2}=\mathcal{U}+\mathcal{S}+\mathcal{W}$ be the corresponding (fiberwise) decomposition into irreducible subspaces. Then this induces for the curvature tensor $R=R^{g}$ a decomposition $R=U+S+W$. For $R=R^{g}=R_{+}+R_{-}$, we denote as in I 1 by $\operatorname{Ric}=\operatorname{Ric}^{g}$ the Ricci tensor, by $\tau=\tau^{g}$ the scalar curvature, by $K=K^{g}$ the sectional curvature and by $W=W^{g}=W_{+}+W_{-}$the Weyl tensor. There are decompositions for the pointwise norms ||$_{x}$ as follows:

$$
\begin{aligned}
& |R|^{2}=\left|R_{+}\right|^{2}+\left|R_{-}\right|^{2}=|U|^{2}+|S|^{2}+|W|^{2}=4\left|W_{+}\right|^{2}+\left|W_{-}\right|^{2}+2|\operatorname{Ric}|^{2}-\frac{1}{3} \tau^{2}, \\
& |\operatorname{Ric}|^{2}=6|U|^{2}+2|S|^{2}, \\
& \tau^{2}=24|U|^{2} .
\end{aligned}
$$

We obtain other decompositions if we consider the curvature operator $R$ as acting from $\Lambda^{2}=\Lambda_{+}^{2} \oplus \Lambda_{-}^{2}$ to $\Lambda_{+}^{2} \oplus \Lambda_{-}^{2}$, for an orthonormal basis $e_{1}, e_{2}, e_{3}, e_{4}$

$$
R\left(e_{i} \wedge e_{j}\right)=\frac{1}{2} \sum R_{i j k l} e_{k} \wedge e_{l}=\Omega_{i j}
$$

$\Omega=\left(\Omega_{i j}\right)=$ matrix of curvature forms, $\Omega_{i j}\left(e_{k}, e_{l}\right)=R_{i j k l}$. We can write $R$ with respect to the orthogonal basis $e_{1} \wedge e_{2}+e_{3} \wedge e_{4}, e_{1} \wedge e_{4}+e_{2} \wedge e_{3}, e_{1} \wedge e_{3}+e_{2} \wedge e_{4}$ in $\Lambda_{+}^{2}$, $e_{1} \wedge e_{3}+e_{2} \wedge e_{4}, e_{1} \wedge e_{2}-e_{3} \wedge e_{4}, e_{1} \wedge e_{4}-e_{2} \wedge e_{3}$ in $\Lambda_{-}^{2}$, as

$$
R=\left(\begin{array}{ll}
A & B \\
C & D
\end{array}\right)
$$

with $A=A^{*}, C=B^{*}, D=D^{*}, \operatorname{tr} A=\operatorname{tr} D=\frac{\tau}{4}, B=\operatorname{Ric}-\frac{1}{4} \tau g$ and $\left(\begin{array}{cc}A & 0 \\ 0 & D\end{array}\right)-\frac{\tau}{12}=W$, $W^{+}=A-\frac{\tau}{12}, W^{-}=D-\frac{\tau}{12}$. We obtain for the first Pontrjagin form $p_{1}$

$$
\begin{aligned}
p_{1} & =-\frac{1}{8 \pi^{2}} \operatorname{tr}(R \wedge R)=-\frac{1}{8 \pi^{2}} \operatorname{tr}(A \wedge A)+\operatorname{tr}(D \wedge D) \\
& =-\frac{1}{8 \pi^{2}}(-2)\left(\left|W_{+}\right|^{2}-\left|W_{-}\right|^{2}\right) \mathrm{dvol}=\frac{1}{4 \pi^{2}}\left(\left|W_{+}\right|^{2}-\left|W_{-}\right|^{2}\right) \mathrm{dvol} \\
& =\frac{1}{12 \pi^{2}}\left(\left|R_{+}\right|^{2}-\left|R_{-}\right|^{2}\right) \mathrm{dvol}
\end{aligned}
$$

and for $\sigma\left(M^{4}, g\right)=\int L(g)=\int \frac{1}{3} p_{1}=\frac{1}{12 \pi^{2}} \int\left(\left|W_{+}\right|^{2}-\left|W_{-}\right|^{2}\right)$ dvol. Assuming $g \in$ ${ }^{b, 2} \mathcal{M}^{1,2}\left(B_{0}, 1, f\right), \sigma\left(M^{4}, g\right)$ is well defined. The Euler form $E(g)$ has the representation

$$
\begin{aligned}
E(g) & =\frac{1}{8 \pi^{2}} \operatorname{tr}(* R)^{2} \mathrm{dvol}=\frac{1}{8 \pi^{2}}\left(|U|^{2}-|S|^{2}+|W|^{2}\right) \mathrm{dvol} \\
& =\frac{1}{8 \pi^{2}} \operatorname{tr}\left(A^{2}-2 B B^{*}+D^{2}\right) \mathrm{dvol}=\frac{1}{32 \pi^{2}}\left(|R|^{2}-4|\mathrm{Ric}|^{2}+\tau^{2}\right) \mathrm{dvol} .
\end{aligned}
$$

For $g \in{ }^{b, 2} \mathcal{M}^{1,2}\left(B_{0}, 1, f\right), \int E(g)=\chi(M, g)$ is well defined. Hence we obtain

Proposition 2.39. Let $\left(M^{4}, g\right)$ be open, complete, oriented and $g \in{ }^{b, 2} \mathcal{M}^{1,2}\left(B_{0}, 1, f\right)$. Then $\sigma(M, g)$ and $\chi(M, g)$ are well defined and an invariant of $\operatorname{comp}(g)$.

REMARK 2.40. According to (2.46)-(2.48), $\int\left|R^{g}\right|^{2}$ dvol $<\infty$ would be sufficient for the existence of $\sigma(M, g)$ and $\chi(M, g)$. But this condition would not establish a uniform strucutre, we would not have components and invariance properties (where we used in 
particular Gaffney's theorem). Moreover, we need the bounded curvature property for the connection with the theorems 2.37, 2.38.

We obtain from proposition 2.39 and its proof the simple

Corollary 2.41. If $\left(M^{4}, g\right)$ is additionally Einstein then $\chi(M, g) \geq 0$ and $\left|\sigma\left(M^{4}, g\right)\right|$ $\leq \frac{2}{3} \chi\left(M^{4}, g\right)$. Moreover, $\chi\left(M^{4}, g\right)=0$ if and only if $\left(M^{4}, g\right)$ is flat.

Proof. If $\left(M^{4}, g\right)$ is Einstein then $S \equiv 0, B \equiv 0$ and $\frac{1}{12 \pi^{2}}\left(\left|W_{+}\right|^{2}-\left|W_{-}\right|^{2}\right) \leq$ $\frac{2}{3} \frac{1}{8 \pi^{2}}\left(|U|^{2}+\left|W_{+}\right|^{2}-\left|W_{-}\right|^{2}\right)$. Hence $\sigma\left(M^{4}, g\right) \leq \frac{2}{3} \chi(M, g)$. Changing the orientation replaces $\sigma\left(M^{4}, g\right)$ by $-\sigma\left(M^{4}, g\right)$ and we get altogether $\left|\sigma\left(M^{4}, g\right)\right| \leq \frac{2}{3} \chi\left(M^{4}, g\right)$

The same estimate holds for $\frac{2}{3}$-pinched Ricci curvature.

Proposition 2.42. Suppose the hypotheses of 2.39 and additionally that the Ricci curvature of $\left(M^{4}, g\right)$ is negative and $\frac{2}{3}$-pinched, i.e. there exists $A>0$ s.t.

$$
-A g \leq \text { Ric } \leq-\frac{2}{3} A g \text {. }
$$

Then for all $g^{\prime} \in \operatorname{comp}(g) \subset{ }^{b, 2} \mathcal{M}^{1,2}\left(B_{0}, 1, f\right)$,

$$
\left|\sigma\left(M^{4}, g^{\prime}\right)\right| \leq \frac{2}{3} \chi\left(M^{4}, g^{\prime}\right)
$$

Proof. We have

$$
\left|\sigma\left(M^{4}, g\right)\right|=\left|\int L(g)\right| \leq \int|L(g)| \mathrm{dvol}=\frac{1}{12 \pi^{2}} \int\left(\left|W_{+}\right|^{2}+\left|W_{-}\right|^{2}\right) \mathrm{dvol}
$$

and

$$
\chi\left(M^{4}, g\right)=\int E(g)=\frac{1}{8 \pi^{2}} \int\left(|U|^{2}-|S|^{2}+|W|^{2}\right) \mathrm{dvol} .
$$

Sufficient for (2.50) would be $|S|^{2} \leq|U|^{2}$ and sufficient for this is (2.49) as pointed out by $[21]$.

EXAMPLES 2.43. 1) Examples for 2.39 with infinite volume are e.g. manifolds $M^{4}$ of the smooth type $M^{4}=M_{0}^{4} \cup \partial M_{0}^{4} \times\left[0, \infty\right.$ [ where the curvature at the cylinder $\partial M_{0}^{4} \times[0, \infty[$ is bounded and asymptotically flat in the sense $\int_{\partial M_{0}^{4} \times[0, \infty[}|R|$ dvol $<\infty$. This can be easily realized by warped product metrics.

2) Examples for 2.39, 2.41, 2.42 with finite volume are given by hyperbolic 4-manifolds of finite volume.

3) Generalizations of these examples are given by variation of $g$ inside $\operatorname{comp}(g)$.

TheOREM 2.44. Let $\left(M^{4}, g\right)$ be open, complete, $\operatorname{vol}\left(M^{4}, g\right)<\infty,|K| \leq 1$ and suppose that $\left(M^{4}, g\right)$ admits a normal covering $(\tilde{M}, g)$ satisfying $\operatorname{geo}(\tilde{M}) \leq 1$.

a) If $\chi\left(M^{4}, g\right)<0$ then $M^{4}$ does not admit a complete Einstein metric $g^{\prime}$ satisfying $\operatorname{vol}\left(M^{4}, g^{\prime}\right)<\infty,\left|K_{g^{\prime}}\right| \leq 1$, geo $\left(M^{\tilde{4}}, g^{\prime}\right) \leq 1$ for some normal covering.

b) If $\chi\left(M^{4}, g\right)>0$ and $\left|\sigma\left(M^{4}\right)\right|>\frac{2}{3} \chi\left(M^{4}, g\right)$ then $M^{4}$ does not admit a complete Einstein metric $g^{\prime}$, s.t. $\operatorname{vol}\left(M^{4}, g^{\prime}\right)<\infty,\left|K g^{\prime}\right| \leq 1$, geo $\left(M^{\tilde{4}}, g^{\prime}\right) \leq 1$. Moreover, there does not exist a complete metric $g^{\prime}$ satisfying

$$
-A g^{\prime} \leq \operatorname{Ric}\left(g^{\prime}\right) \leq-\frac{2}{3} A g^{\prime}
$$

and $\left|K g^{\prime}\right| \leq 1, \operatorname{vol}\left(M^{4}, g^{\prime}\right)<\infty$ and $\operatorname{geo}\left(M^{\tilde{4}}, g^{\prime}\right) \leq 1$ for some normal covering. 
Proof. a) Suppose the existence of an Einstein metric $g^{\prime}$ with the required properties. Then $\chi\left(M^{4}, g\right), \chi\left(M^{4}, g^{\prime}\right)$ are well defined. $\chi\left(M^{4}, g\right)=\chi\left(M^{4}, g^{\prime}\right)$, according to theorem $2.38 \mathrm{~b})$. But this contradicts $\chi\left(M^{4}, g^{\prime}\right)=\frac{1}{8 \pi^{2}} \int\left(|U|^{2}+|W|^{2}\right)$ dvol $\geq 0$. b) and c): Quite analogously we derive by means of theorem $2.38 \mathrm{~b}$ ), corollary 2.41 and proposition 2.42 a contradiction.

Until now we defined characteristic numbers in the following cases:

1) $R \in L_{1}$ and bounded, $\operatorname{vol}(M)$ arbitrary,

2) $R$ bounded, $\operatorname{vol}(M)<\infty$.

There remains the case $R$ bounded, $\operatorname{vol}(M)=\infty$. It is clear that in this case we will not get characteristic numbers by integration. $\left(M^{n}, g\right)$ is called closed at infinity if for any $\varphi \in C(M), 0<A^{-1}<\varphi<A, A>0$ some constant, the form $\varphi \cdot$ dvol generates a nontrivial cohomology class in ${ }^{b} H^{n}\left(M^{n}, g\right)$. A fundamental class for $M$ is a positive continuous linear function $\mathfrak{m}:{ }^{b} \Omega^{n}(M) \rightarrow \mathbb{R}$ such that $\langle\mathfrak{m}, \operatorname{dvol}\rangle \neq 0$ and $\langle\mathfrak{m}, d \psi\rangle=0$.

Proposition 2.45. $M$ has a fundamental class if and only if $M$ is closed at infinity.

Proof. Write $\mathcal{L}(\mathrm{dvol})$ for the linear hull of dvol, $0 \notin[\mathrm{dvol}] \in{ }^{b} \bar{H}^{n}(M)$ and set $\langle\mathfrak{m}$, dvol $\rangle=1$, $\left.\mathfrak{m}\right|_{{ }^{b} B^{n}} \equiv 0$. Then we obtain by linear extension $\mathfrak{m}$ on $\mathcal{L}(\mathrm{dvol}) \oplus{ }^{b} \bar{B}^{n}$ as positive continuous linear functional. The Hahn-Banach theorem for the extension of such functionals yields the desired $\mathfrak{m}$. The other direction is absolutely trivial.

Define the penumbra for $K \subset M$ by

$$
\operatorname{Pen}^{+}(K, r)=\mathrm{CL}\left(\bigcup_{x \in K} B_{r}(x)\right), \quad \operatorname{Pen}^{-}(K, r)=\mathrm{CL}\left(M \backslash \operatorname{Pen}^{+}(M \backslash K, r)\right) .
$$

We call an exhaustion $M_{1} \subset M_{2} \subset \cdots, \bigcup_{i} M_{i}=M$, by compact submanifolds a regular exhaustion if for each $r \geq 0$

$$
\lim _{i \rightarrow \infty} \operatorname{vol}\left(\operatorname{Pen}^{+}\left(M_{i}, r\right)\right) / \operatorname{vol}\left(\operatorname{Pen}^{-}\left(M_{i}, r\right)\right)=1 .
$$

It is clear that then automatically

$$
\lim _{i \rightarrow \infty} \operatorname{vol}\left(\operatorname{Pen}^{+}\left(M_{i}, r\right)\right) / \operatorname{vol}\left(M_{i}\right)=1, \quad \lim _{i \rightarrow \infty} \operatorname{vol}\left(M_{i}\right) / \operatorname{vol}\left(\operatorname{Pen}^{-}\left(M_{i}, r\right)\right)=1 .
$$

EXAMPLES 2.46. 1) $\left(M^{n}, g\right)=\left(\mathbb{R}^{n}, g_{\text {standard }}\right)$ admits a regular exhaustion.

2) Any $\left(M^{n}, g\right)$ with subexponential growth admits a regular exhaustion.

3) The hyperbolic space admits no regular exhaustion.

Let $\left\{M_{i}\right\}_{i \geq 1}$ be a regular exhaustion and set for $\omega \in{ }^{b} \Omega^{n}$

$$
\left\langle\mathfrak{m}_{i}, \omega\right\rangle:=\frac{1}{\operatorname{vol}\left(M_{i}\right)} \int_{M_{i}} \omega .
$$

Then $\left|\left\langle\mathfrak{m}_{i}, \omega\right\rangle\right| \leq \sup _{x}|\omega|_{x}={ }^{b}|\omega|$, i.e. $\left|\mathfrak{m}_{i}\right| \leq 1$, the $\mathfrak{m}_{i}$ belong to the unit ball in $\left({ }^{b} \Omega^{n}\right)^{*}$. This unit ball is compact in the weak star topology, according to the Banach-Alaoglu theorem, hence the sequence $\left\{\mathfrak{m}_{i}\right\}_{i}$ has a weak star limit point $\mathfrak{m}$. $\mathfrak{m}$ is then called associated to the regular exhaustion $\left\{M_{i}\right\}_{i}$.

Proposition 2.47. Let $\mathfrak{m}$ be associated to a regular exhaustion $\left\{M_{i}\right\}_{i}$. Then $\mathfrak{m}$ is a fundamental class for $M$. 
Proof. There remains only to show $\langle\mathfrak{m}, d \psi\rangle=0$. Let $\Phi_{i} \in C^{\infty}(M)$ such that $0 \leq \Phi_{i}(x) \leq 1$, $\Phi_{i}=1$ on $M_{i}, \Phi_{i}=0$ outside $\operatorname{Pen}^{+}\left(M_{i}, 1\right),\left|\nabla \Phi_{i}\right| \leq 2$. We obtain for $\omega \in{ }^{b} \Omega^{n}$

$$
\left|\int_{M_{i}} \omega-\int_{M} \Phi_{i} \omega\right| \leq\left(\operatorname{vol}\left(\operatorname{Pen}^{+}\left(M_{i}, 1\right)\right)-\operatorname{vol}\left(M_{i}\right)\right)^{b}|\omega|,
$$

hence

$$
\lim _{i \rightarrow \infty} \frac{1}{\operatorname{vol}\left(M_{i}\right)}\left(\int_{M_{i}} \omega-\int_{M} \Phi_{i} \omega\right)=0 .
$$

Therefore we would be done if we could show

$$
\lim _{i \rightarrow \infty} \frac{1}{\operatorname{vol}\left(M_{i}\right)} \int_{M} \Phi_{i} d \psi=0
$$

Integration by parts yields

$$
\begin{aligned}
\int \Phi_{i} d \psi & =-\int d \Phi_{i} \wedge \psi \\
\left|\int \Phi_{i} d \psi\right| & =\left|\int d \Phi_{i} \wedge \psi\right| \leq 2\left(\operatorname{vol}\left(\operatorname{Pen}^{+}\left(M_{i}, 1\right)\right)-\operatorname{vol}\left(M_{i}\right)\right)^{b}|\psi|
\end{aligned}
$$

which implies the assertion.

Define for $\omega \in{ }^{b, 1} \mathcal{C}_{p}\left(B_{0}\right),\left[Q_{i_{1} \ldots i_{k}}(\omega)\right] \in{ }^{b} H^{n}(M)$ a (bounded) characteristic class and a regular exhaustion $\left\{M_{i}\right\}_{i}$ with associated fundamental class $\mathfrak{m}$ the characteristic number

$$
Q_{i_{1} \ldots i_{k}}(P, \operatorname{comp}(\omega))[\mathfrak{m}]:=\left\langle\mathfrak{m},\left[Q_{i_{1} \ldots i_{k}}\right]\right\rangle:=\lim _{i \rightarrow \infty} \frac{1}{\operatorname{vol}\left(M_{i}\right)}\left(Q_{i_{1} \ldots i_{k}}\right) .
$$

Then, according to proposition $2.47, Q_{i_{1} \ldots i_{k}}(P, \operatorname{comp}(\omega))[\mathfrak{m}]$ is well defined. In particular we obtain in this case average Euler numbers, average signatures, which are special cases of Roe's (average) topological index. Average characteristic numbers are also considered in [17], [18], [15]. Some simple geometric examples are calculated in [17].

In all cases discussed until now, we restricted to the case of connections (or metrics) with finite $p$-action or bounded curvature or both. The next proposition shows that this is in fact a restriction.

Proposition 2.48. Let $\left(M^{n}, g\right)$ be open, complete, satisfying $(I), G$ a compact Lie group, $P=P(M, G)$ a $G$-principal fibre bundle, $\varrho: G \rightarrow U(N)$ resp. $O(N)$ a faithful representation, $E$ the associated vector bundle, $p \leq 1$. Then there exist $G$-connections $\omega$ such that their p-action is infinite or the curvature is unbounded or both, respectively.

Proof. Consider the closed unit ball $\bar{B}_{1}(0) \subset \mathbb{R}^{n}$ and set up in $\bar{B}_{1}(0)$ constant 1 -forms $\omega_{i j}, \omega_{i j}=-\bar{\omega}_{j i}$ or $\omega_{i j}=-\omega_{j i}, 1 \leq i, j \leq N$, respectively, such that some $\Omega_{i j}=d \omega_{i j}-$ $\sum_{k} \omega_{i k} \wedge \omega_{k j}$ are $\neq 0$. Now consider an infinite sequence $U_{\nu}=U_{\varepsilon_{\nu}}\left(x_{\nu}\right)$ of closed geodesic balls with pairwise distance $\geq d>0$, introduce in each geodesic ball normal coordinates $u^{1}, \ldots, u^{n}, \sum_{i}\left(u^{i}\right)^{2} \leq \varepsilon_{\nu}$, choose over $U_{\nu}$ orthonormal bases $e_{1, \nu}, \ldots, e_{N, \nu}$ and define with respect to these bases local connection matrices $\omega_{i j, \nu}^{\prime}$ by $\omega_{i j, \nu}^{\prime}\left(u_{1}, \ldots, u_{n}\right):=\omega_{i j}$. If $\int_{u_{\nu}}\left|\Omega_{i j, \nu}^{\prime}\right|_{x}^{p} \operatorname{dvol}_{x}(g)=a_{\nu} \neq 0$, set $\omega_{i j, \nu}^{\prime \prime}=\left(a_{\nu}+\frac{1}{a_{\nu}}\right)^{\frac{1}{2} p} \omega_{i j, \nu}^{\prime}$. This connection over $\bigcup_{\nu} U_{\nu}$ is smoothly extendable over the whole of $M$ and gives a connection with $\int_{M}\left|\Omega^{\prime \prime}\right|_{x}^{p} \operatorname{dvol}_{x}(g) \geq$ 
$\sum_{\nu} \int_{U_{\nu}}\left|\Omega^{\prime \prime}\right|_{x}^{p} \operatorname{dvol}_{x}(g) \geq \sum_{\nu} 1=\infty$. Setting $\omega_{i j, \nu}^{\prime \prime}=\nu \cdot\left(a_{\nu}+\frac{1}{a_{\nu}}\right)^{\frac{1}{2} p} \cdot \omega_{i j, \nu}^{\prime \prime}$ yields examples for the other cases.

The conditions of finite $p$-action or boundedness can be reformulated in the language of classifying spaces and classifying mappings.

We start with $G=U(N)$. Let $V_{N, k} \stackrel{U(k)}{\longrightarrow} G_{N, k}$ be the Stiefel bundle over the complex Grassmann manifold $G_{N, k}$ of all $k$-subspaces $\subset \mathbb{C}^{N}$ and $S$ the matrix valued function on $V_{N, k}$ defined by $S\left(v_{1}, \ldots, v_{k}\right)=a_{i j}:=\left(b_{i j}\right)^{t}$, where $v_{1}, \ldots, v_{k}$ is a unitary $k$-frame, $e_{1}, \ldots, e_{N}$ the standard base in $\mathbb{C}^{N}$ and $v_{i}=\sum_{j=1}^{N} b_{i j} e_{j}$.

Proposition 2.49. a) $\gamma_{U}=S^{*} d S$ is a $U(N)$-invariant connection form at $V_{N, k}$.

b) Let be $m=(n+1)(2 n+1) k^{3}$. If $P$ is a $U(k)$-principal fibre bundle over a manifold of dimension $\leq n$ and $\omega$ a connection form for $P$, then there exists a smooth bundle morphism $f_{P}: P \rightarrow V_{m, k}=P_{n, U(k)}$ such that $f_{P}^{*} \gamma=\omega$.

We refer to [20], p. 564, 568 for the proof.

$\gamma_{0}$ is called an $n$-universal connection for $U(k)$. In a similar manner one defines on the real Stiefel bundle $V_{m, k}^{r} \stackrel{O(k)}{\longrightarrow} G_{m, r}^{r}$ an $n$-universal $O(k)$-connection $\gamma_{0}^{r}$.

For an arbitrary compact Lie group $G$ one constructs by means of a faithful representation $G \rightarrow O(k)$ an $n$-universal connection $\gamma_{G}$ on the $n$-universal bundle $P_{n, G} \rightarrow B_{n, G}$ (cf. [20], p. 570).

According to proposition 2.49, we refine the bundle concept and consider instead of a bundle $P$ pairs $\left(P, f_{P}\right), f_{P}: P \rightarrow P_{n, G}$ a $C^{1}$-classifying bundle map.

$\left(P, f_{P}\right)$ is called a $(p, f)$-bundle if $f_{p}^{*} \gamma_{G} \in C^{1} \mathcal{C}_{p}(f, p)=\left\{\omega\right.$ a $C^{1}$-connection $\mid$ $\left.\int\left|\Omega^{\omega}\right|_{x}^{p} \operatorname{dvol}_{x}(g)<\infty\right\}$, i.e. $\int\left|\Omega^{f_{p}^{*} \gamma_{G}}\right|_{x}^{p} \operatorname{dvol}_{x}(g)<\infty$. In the same manner we define $\left(P, f_{P}\right)$ to be a $b$-bundle if $f_{p}^{*} \gamma_{G} \in C^{1} \mathcal{C}_{p}\left(B_{0}\right)$, i.e. ${ }^{b}\left|\Omega^{f_{p}^{*} \gamma_{G}}\right|<\infty$.

Most interesting for applications is the case assuming $\left(B_{0}\right)$ and finite $p$-action. Hence we assume $\left(B_{0}\right)$ for $\left(M^{n}, g\right) .\left(P, f_{P}\right)$ is a $(b, p, f)$-bundle, if $f_{p}^{*} \gamma_{G} \in{ }^{b, 1} \mathcal{C}_{p}^{p, 1}\left(B_{0}, f, p\right)$. Two $(b, p, f)$-bundles $\left(P, f_{P}\right),\left(P, f_{P}^{\prime}\right)$ are called equivalent if $f_{P}^{*} \gamma_{G}, f_{P}^{\prime *} \gamma_{G}$ are contained in the same component of ${ }^{b, 1} \mathcal{C}_{P}^{p, 1}\left(B_{0}, f, p\right)$. Assume $G$ to be a subgroup of $U(N), \operatorname{dim} M^{n}=2 k$. At the level of base spaces we consider classifying maps $f_{M}: M \rightarrow B_{n, G}$. A pair $\left(M, f_{M}\right)$ is called a $(p, c)$-bundle if all classes $f_{M}^{*} c_{i_{1} \ldots i_{k}}, i_{1}+\cdots+i_{k}=k$, are elements of $H^{2 k, p}(M)$. $\left(M, f_{M}\right)$ is called a $(b, c)$-bundle if all classes $f_{M}^{*} c_{i_{1} \ldots i_{k}}$ are elements of ${ }^{b} H^{2 k}(M) .\left(M, f_{M}\right)$ is called a $(b, p, c)$-bundle if all classes $f_{M}^{*} c_{i_{1} \ldots i_{k}}$ are elements of ${ }^{b} H^{2 k, p}(M)$. It is clear that a given $f_{P}: P \rightarrow P_{n, G}$ uniquely determines $f_{M}: M \rightarrow B_{n, G}$.

The case $G \subseteq O(N), \operatorname{dim} M=4 k$, is quite parallel. Then we consider the $p_{i_{1} \ldots i_{k}}$, $i_{1}+\cdots+i_{k}=k$ and define $\left(M, f_{M}\right)$ to be a $(p, p o)$-bundle if all classes $f_{M}^{*} p_{i_{1} \ldots i_{k}}$, $i_{1}+\cdots+i_{k}=k$ are elements of $H^{4 k, p}(M)$. Analogously for $(b, p o)$ - and $(b, p, p o)$-bundles $\left(M, f_{M}\right)$.

If we replace $p_{i_{1} \ldots i_{k}}$ by the class of Hirzebruch genus $L_{k}$ then we get the notion of a $\left(p, L_{k}\right)-,\left(b, L_{k}\right)$ - or $\left(b, p, L_{k}\right)$-bundle $\left(M, f_{M}\right)$, respectively.

Theorem 2.50. a) Suppose $G \subset U(N)$, $\operatorname{dim} M=2 k$. $(M, g)$ satisfying $\left(B_{0}\right), p \geq 1$. $A(b, p, f)$-bundle $\left(P, f_{P}\right)$ defines a unique $(b, p)$-bundle $\left(M, f_{M}\right)$. If $\left(P, f_{P}\right),\left(P, f_{P}^{\prime}\right)$ are 
equivalent then $f_{M}^{*} c_{i_{1} \ldots i_{k}}={f^{\prime *}}_{M}^{*} c_{i_{1} \ldots i_{k}}$ for all $c_{i_{1} \ldots i_{k}}, i_{1}+\cdots+i_{k}=k$. If additionally $p=1$ and $(M, g)$ is complete then even the corresponding characteristic numbers coincide.

b) Suppose $G \subseteq O(N), \operatorname{dim} M=4 k,(M, g)$ satisfying $\left(B_{0}\right), p \geq 1 . A(b, p, f)$-bundle $\left(P, f_{P}\right)$ defines a unique $(b, p, p o)$-bundle $\left(M, f_{M}\right)$ which is simultaneously a $\left(b, p, L_{k}\right)$ bundle. If $\left(P, f_{P}\right),\left(P, f_{P}^{\prime}\right)$ are equivalent then $f_{M}^{*} p_{i_{1} \ldots i_{k}}={f^{\prime}}_{M}^{*} p_{i_{1} \ldots i_{k}}$ and $f_{M}^{*} L_{k}=$ $f^{\prime *}{ }_{M} L_{k}$. If additionally $p=1$ and $(M, g)$ is complete then the corresponding characteristic numbers coincide.

The proof follows immediately from the definitions and theorem 2.14.

EXAMPLE 2.51. It is possible that ${ }^{b, 1} \mathcal{C}_{p}^{1,1}\left(B_{0}, 1, f\right)=\emptyset$. Let $\left(M^{2}, g\right)$ be an infinitely connected open complete Riemannian manifold with bounded sectional curvature $K$, $K=K_{+}-K_{-}$,

$$
K_{+}=\left\{\begin{array}{cc}
K, & K \geq 0, \\
0, & K<0,
\end{array} \quad K_{-}=\left\{\begin{array}{cc}
-K, & K \leq 0 \\
0, & K>0
\end{array}\right.\right.
$$

Then $\int K_{-}$dvol $=\infty$ (cf. [16], theorem 13). In particular $\int|K|$ dvol $=\infty$ which implies $\int\left|\Omega^{\omega}(g)\right|$ dvol $=\infty$. The proof essentially relies on the Gauß-Bonnet theorem (as one would expect) for compact surfaces. But this theorem holds for any metrizable connection in the orthogonal 2-frame bundle $P\left(M^{2}, O(2)\right)$ over $M^{2}$ ([19], p. 305/306). The sectional curvature $K$ is defined by $\Omega_{1,2}=K$ dvol. As conclusion we obtain ${ }^{b, 1} \mathcal{C}_{p}\left(B_{0}, 1, f\right)=\emptyset$.

3. Combinatorial characteristic numbers. Let $\left(M^{n}, g\right)$ be open, complete, oriented. We consider triangulations $T:|K| \stackrel{\cong}{\longrightarrow} M$. Let $\sigma^{n}$ be a curved $n$-simplex in $M^{n}$. We define the fullness $\theta(\sigma)$ by $\theta(\sigma):=\operatorname{vol}(\sigma) /(\operatorname{diam}(\sigma))^{n}$, where $\operatorname{vol}(\sigma)$, $\operatorname{diam}(\sigma)$ means volume and diameter with respect to $g . T:|K| \stackrel{\cong}{\longrightarrow} M$ will be called uniform if it satisfies the following conditions:

a) There exists a $\theta_{0}>0$ such that for every curved simplex $\sigma^{n}$ the fullness satisfies the inequality $\theta(\sigma) \geq \theta_{0}$.

b) There exist constants $c_{1}>c_{2}>0$ such that for every $\sigma^{n}$ we have

$$
c_{2} \leq \operatorname{vol}(\sigma) \leq c_{1} .
$$

c) There exists a constant $c>0$ such that for every vertex $\nu \in K$ the barycentric coordinate function $\varphi_{\nu}: M \rightarrow R$ satisfies the condition $\left|\nabla \varphi_{\nu}\right| \leq c$.

If one assumes a), then $\mathrm{b}$ ) is equivalent to the existence of bounds $d_{1}>d_{2}>0$ with $d_{2} \leq \operatorname{diam}(\sigma) \leq d_{1}$ for all $\sigma \in K$. a) and b) are equivalent to the boundedness of the volumes from below and the diameters from above.

Consider the Whitney transformation $W$,

$$
\sigma^{q} \rightarrow W\left(\sigma^{q}\right)=\omega_{\sigma}:=q ! \sum_{i=0}^{q}(-1)^{i} \varphi_{i} d \varphi_{0} \wedge \cdots \wedge \widehat{d \varphi_{i}} \wedge \cdots \wedge d \varphi_{q},
$$

$\varphi_{i}$ the barycentric coordinates.

THEOREM 3.1. If $T:|K| \rightarrow\left(M^{n}, g\right)$ is uniform then $W$ induces topological isomorphisms

$$
H^{*, p}(K) \rightarrow H_{d R}^{*, p}(M), \quad \bar{H}^{*, p}(K) \rightarrow \bar{H}_{d R}^{*, p}(M) .
$$

We refer to [13] for the proof. 
The proof of 3.1 in [13] is performed even under weaker assumptions, $K$ uniformly locally finite and $|d T|,\left|d T^{-1}\right| \leq C$.

The point is the multiplicativity which is settled by

Theorem 3.2. Let $\left[z^{q}\right] \in H^{q, p}(K),\left[z^{\prime n-q}\right] \in H^{n-q, r}(K), \frac{1}{p}+\frac{1}{r}=1$. Then

$$
\int_{M} W(z) \wedge W\left(z^{\prime}\right)=C(n, q)\left\langle\left[z^{q}\right] \cup\left[z^{\prime n-q}\right],[K]\right\rangle .
$$

We refer to [8], [10] for the meaning of the r.h.s. of (3.1) and the proof of 3.2.

In the case of $n=4 k, p=r=2$, iteration of (3.1) yields

Corollary 3.3. If $\left(M^{4 k}, g\right)$ is open, oriented, $g \in{ }^{b, 2} \mathcal{M}^{2,2}\left(B_{0}, 2, f\right)$ and satisfies the condition $r_{\mathrm{inj}}(M, g)>0(I)$, then $\left(M^{n}, g\right)$ admits uniform triangulations $T:|K| \stackrel{\cong}{\longrightarrow} M$ and

$$
p_{i_{1} \ldots i_{k}, c}=C(4 k) p_{i_{1} \ldots i_{k}, a},
$$

where $p_{i_{1} \ldots i_{k}, c}$ or $p_{i_{1} \ldots i_{k}, a}$ are the combinatorial or analytical Pontrjagin numbers, respectively.

4. Bordism and relative characteristic numbers. We consider oriented open manifolds $\left(M^{n}, g\right)$ satisfying

$$
\left|\nabla^{i} R\right| \leq C_{i}, \quad i=0,1,2, \ldots, k
$$

and

$$
r_{\text {inj }}(M, g) \equiv \inf r_{\text {inj }}(g, x)>0 .
$$

$\left(B^{n+1}, g_{B}\right)$ is a bordism between $\left(M_{1}^{n}, g_{1}\right)$ and $\left(M_{2}^{n}, g_{2}\right)$ if it satisfies the following conditions.

1) $\left(\partial B,\left.g_{B}\right|_{\partial B}\right) \cong\left(M_{1}, g_{1}\right) \cup\left(-M_{2}, g_{2}\right)$,

2) there exists $\delta>0$ such that $\left.g_{B}\right|_{U_{\delta}(\partial B)} \cong g_{\partial B}+d t^{2}$,

3) $\left(B, g_{B}\right)$ satisfies $\left(B_{k}\right)$ and $\inf _{x \in B \backslash U_{\delta}(\partial B)} r_{\text {inj }}\left(g_{B}, x\right)>0$,

4) there exists $R>0$ such that $B \subset U_{R}\left(M_{1}\right), B \subset U_{R}\left(M_{2}\right)$.

We denote $\left(M_{1}, g_{1}\right) \underset{b}{\sim}\left(M_{2}, g_{2}\right)$. $\left(B^{n+1}, g_{B}\right)$ is called a bordism. Sometimes we denote additionally $\underset{b, b_{g}}{\sim}, b_{g}$ stands for bounded geometry, i.e. $(I)$ and $\left(B_{k}\right)$.

LEMMA 4.1. a) $\underset{b}{\sim}$ is an equivalence relation. Denote by $\left[M^{n}, g\right]$ the bordism class.

b) $\left[M \cup M^{\prime}, g \cup g^{\prime}\right]=\left[M \# M^{\prime}, g \# g^{\prime}\right]$.

c) Set $[M, g]+\left[M^{\prime}, g^{\prime}\right]:=\left[M \cup M^{\prime}, g \cup g^{\prime}\right]=\left[M \# M^{\prime}, g \# g^{\prime}\right]$. Then + is well defined and the set of all $\left[M^{n}, g\right]$ becomes an abelian semigroup.

Denote by $\Omega_{n}^{n c}$ the corresponding Grothendieck group. Similarly one defines $\Omega_{n}^{n c}(X)$ generated by pairs $\left(\left(M^{n}, g\right), f: M^{n} \rightarrow X\right), f$ bounded and uniformly proper.

Remarks 4.2. 1) Condition 4) above looks like $d_{G H}\left(M, M^{\prime}\right) \leq R$, where $d_{G H}$ is the Gromov-Hausdorff distance (cf. [9]). But this is wrong.

2) There is no chance to calculate $\Omega_{n}^{n c}$.

3) One would like to have a geometric representative for 0 and for $-[M, g]$. 
The way out from this is to establish bordism theory for special classes of open manifolds or/and further restrictions on bordism.

Our first example is bordism with compact support. Here condition 1) above remains but one replaces 2)-4) by the condition

There exists a compact submanifold $C^{n+1} \subset B^{n+1}$

such that $\left(\overline{B \backslash C},\left.g_{B}\right|_{B \backslash C}\right)$ is a product bordism, i.e.

$$
\left(\overline{B \backslash C}, g_{\overline{B \backslash C}}\right) \cong\left(\overline{M \backslash C} \times[0,1], g_{\overline{M \backslash C}}+d t^{2}\right) \text {. }
$$

We write $\underset{b, c s}{\sim}$. Then one gets a bordism group $\Omega_{n}^{n c}(c s)$ (= Grothendieck group).

At a first glance, the calculation of $\Omega_{n}^{n c}(c s)$ or at least the characterization of the bordism classes seems to be very difficult. But we will see that this is not the case. For this, we introduce still some uniform, structures. Denote by $\mathfrak{M}^{n}(m f):=\mathfrak{M}^{n}(m f, n c) \subset \mathfrak{M}_{L}$ the set of isometry classes of complete, open, oriented Riemannian manifolds. Consider pairs $\left(M_{1}^{n}, g_{1}\right),\left(M_{2}^{n}, g_{2}\right) \in \mathfrak{M}^{n}(m f)$ with the following property:

There exist compact submanifolds $K_{1}^{n} \subset M_{1}^{n}$ and $K_{2}^{n} \subset M_{2}^{n}$

and an isometry $M_{1} \backslash K_{1} \stackrel{\Phi}{\longrightarrow} M_{2} \backslash K_{2}$.

For such pairs, we define

$$
\begin{aligned}
& { }^{b} d_{L, \text { iso,rel }}\left(\left(M_{1}, g_{1}\right),\left(M_{2}, g_{2}\right)\right):= \\
& \inf \left\{\max \left\{0, \log { }^{b}|d f|\right\}+\max \left\{0, \log { }^{b}|d h|\right\}+\sup _{x \in M_{1}} \operatorname{dist}(x, h f x)+\sup _{y \in M_{2}} \operatorname{dist}(y, f h y) \mid\right. \\
& f \in C^{\infty}\left(M_{1}, M_{2}\right), g \in C^{\infty}\left(M_{2}, M_{1}\right), \text { and }
\end{aligned}
$$

for some $K_{1} \subset K,\left.f\right|_{M_{1} \backslash K_{1}}$ is an isometry and $\left.\left.g\right|_{f\left(M_{1} \backslash K\right.}=f^{-1}\right\}$.

If $\left(M_{1}, g_{1}\right)$ and $\left(M_{2}, g_{2}\right)$ do not satisfy $(4.1)$, then we define ${ }^{b} d_{L, \text { iso,rel }}\left(\left(M_{1}, g_{1}\right),\left(M_{2}, g_{2}\right)\right)$ $=\infty$. We have ${ }^{b} d_{L, \text { iso,rel }}\left(\left(M_{1}, g_{1}\right),\left(M_{2}, g_{2}\right)\right)=0$ if $\left(M_{1}, g_{1}\right)$ and $\left(M_{2}, g_{2}\right)$ are isometric.

REMARKS 4.3. 1) The notions of Riemannian isometry and distance isometry coincide for Riemannian manifolds. Furthermore, if $f$ is an isometry $f$, then we have ${ }^{b}|d f|=1$.

2) Any $f$ that occurs in the definition of $d_{L, \text { iso,rel }}$ is automatically an element of $C^{\infty, m}\left(M_{1}, M_{2}\right)$ for all $m$. The same holds true for $g$.

We write $\mathfrak{M}_{L, \text { iso,rel }}^{n}(m f)=\mathfrak{M}^{n}(m f) / \sim$ where by definition $\left(M_{1}, g_{1}\right) \sim\left(M_{2}, g_{2}\right)$ if ${ }^{b} d_{L, \text { iso,rel }}\left(\left(M_{1}, g_{1}\right),\left(M_{2}, g_{2}\right)\right)=0$. Set

$$
\left.V_{\delta}=\left\{\left(\left(M_{1}, g_{1}\right),\left(M_{2}, g_{2}\right)\right) \in(\mathfrak{M})_{L, \text { iso,rel }}^{2}(m f)\right)^{2} \mid{ }^{b} d_{L, \text { iso,rel }}\left(\left(M_{1}, g_{1}\right),\left(M_{2}, g_{2}\right)\right)<\delta\right\} .
$$

Proposition 4.4. $\mathcal{L}=\left\{V_{\delta}\right\}_{\delta>0}$ is a basis for a metrizable uniform structure $\mathcal{U}_{L, \text { iso,rel }}$.

Denote by ${ }^{b} \mathfrak{M}_{L, \text { iso,rel }}^{n}(m f)$ the corresponding uniform space.

Proposition 4.5. If $r_{i n j}\left(M_{i}, g_{i}\right)=r_{i}>0, r=\min \left\{r_{1}, r_{2}\right\}$ and ${ }^{b} d_{L, \text { iso,rel }}\left(\left(M_{1}, g_{1}\right)\right.$, $\left.\left(M_{2}, g_{2}\right)\right)<r$ then $M_{1}$ and $M_{2}$ are (uniformly proper) bi-Lipschitz homotopy equivalent.

COROLlaRY 4.6. If we restrict ourselves to open manifolds with injectivity radius $\geq r$, then manifolds $\left(M_{1}, g_{1}\right)$ and $\left(M_{2}, g_{2}\right)$ with ${ }^{b} d_{L, \text { iso,rel-distance less than } r \text { are automatically }}$ (uniformly proper) bi-Lipschitz homotopy equivalent. 
REMARK 4.7. If $\left(M_{1}, g_{1}\right)$ satisfies $(I)$ or $(I)$ and $\left(B_{k}\right)$ and $\left.{ }^{b} d_{L, \text { iso }, \text { rel }}\left(M_{1}, g_{1}\right),\left(M_{2}, g_{2}\right)\right)$ $<\infty$ then $\left(M_{2}, g_{2}\right)$ also satifies $(I)$ or $(I)$ and $\left(B_{k}\right)$.

We cannot show that $b_{\mathfrak{M}_{L, \text { iso,rel }}^{n}}^{n}$ is locally arcwise connected, that components are arc components and ${ }^{b} \operatorname{comp}_{L, \text { iso,rel }}(M, g)=\left\{\left.\left(M^{\prime}, g^{\prime}\right)\right|^{b} d_{L, \text { iso, rel }}\left((M, g),\left(M^{\prime}, g^{\prime}\right)\right)<\infty\right\}$ is wrong. The reason is that we cannot connect non-homotopy-equivalent manifolds by a continuous family of manifolds. A parametrization of nontrivial surgery always contains bifurcation levels where we leave the category of manifolds. A very simple case comes from corollary 4.6 .

COROLlaRY 4.8. If we restrict $\boldsymbol{b}_{L, \text { iso,rel }}$ to open manifolds with injectivity radius $\geq r>0$, then the manifolds in each arc component of this subspace are bi-Lipschitz homotopy equivalent.

Proof. This subspace is locally arcwise connected and components are arc components. Consider an (arc) component and two elements $\left(M_{1}, g_{1}\right)$ and $\left(M_{2}, g_{2}\right)$ of it, connect them by an arc, cover this arc by sufficiently small balls, and apply 4.6 .

By definition, we have

$$
{ }^{b} d_{L, \text { iso,rel }}\left(\left(M_{1}, g_{1}\right),\left(M_{2}, g_{2}\right)\right)<\infty \Rightarrow d_{L}\left(\left(M_{1}, g_{1}\right),\left(M_{2}, g_{2}\right)\right)<\infty,
$$

where $d_{L}$ is the Lipschitz distance of [9]. Hence, $\left(M_{2}, g_{2}\right) \in \operatorname{comp}_{L}\left(M_{1}, g_{1}\right)$, i.e.

$$
\left\{\left.\left(M_{2}, g_{2}\right) \in \mathfrak{M}^{n}(m f)\right|^{b} d_{L, \text { iso,rel }}\left(\left(M_{1}, g_{1}\right),\left(M_{2}, g_{2}\right)\right)<\infty\right\} \subseteq \operatorname{comp}_{L}\left(M_{1}, g_{1}\right) .
$$

For this reason, we denote the left hand side $\{\ldots\}$ of $(4.2)$ by gen ${ }^{b} \operatorname{comp}_{L, \text { iso,rel }}\left(M_{1}, g_{1}\right)=$ $\{\ldots\}=\{\ldots\} \cap \operatorname{comp}_{L}\left(M_{1}, g_{1}\right)$, keeping in mind that this is not an arc component, but a subset of (manifolds in) a Lipschitz arc component.

If we fix $\left(M_{1}, g_{1}\right)$, then in a special case, we have a good overview of the elements in gen ${ }^{b} \operatorname{comp}_{L, \text { iso,rel }}\left(M_{1}, g_{1}\right)$.

EXAMPLE 4.9. Let $\left(M_{1}, g_{1}\right)=\left(\mathbb{R}^{n}, g_{\text {standard }}\right)$. Then gen ${ }^{b} \operatorname{comp}_{L, \text { iso }, \text { rel }}\left(M_{1}, g_{1}\right)$ is in a 1-1 correspondence with $\left\{\left(M^{n}, g\right) \mid M^{n}\right.$ is a closed manifold and $g$ is flat in an annulus contained in a disk neighborhood of a point $\}$.

This can be generalized as follows.

TheOrEm 4.10. Any component gen ${ }^{b} \operatorname{comp}_{L, \text { iso,rel }}(M, g)$ contains at most countably many diffeomorphism types.

Proof. Fix $(M, g) \in$ gen ${ }^{b} \operatorname{comp}_{L, \text { iso,rel }}(M, g)$ and an exhaustion $K_{1} \subset K_{2} \subset \ldots$, $\bigcup K_{1}=M$, of $M$ by compact submanifolds, and let $\left(M^{\prime}, g^{\prime}\right) \in{ }^{b} \operatorname{comp}_{L, \text { iso, rel }}(M, g)$. Then there are $K^{\prime} \subset M^{\prime}$ and $K_{i} \subset M$ such that $M \backslash K_{i}$ and $M^{\prime} \backslash K^{\prime}$ are isometric. The diffeomorphism type of $M^{\prime}$ is completely determined by that of the pair $\left(K_{1} \bigcup_{\partial K_{1}} \cong \partial K^{\prime} K^{\prime}, K_{1}\right)$, but the set of types of such pairs (after fixing $M$ and $K_{1} \subset K_{2} \subset \ldots$ ) is at most countable.

Thus, after fixing $(M, g)$, the diffeomorphism classification of the elements in ${ }^{b}$ comp $_{L, \text { iso,rel }}(M, g)$ seems to be reduced to a "handy" countable discrete problem. This is in fact the case in a sense which is parallel to the classification of compact manifolds. 
Now we connect the calculation of $\Omega_{n}^{n c}(c s)$ with the generalized components gen ${ }^{b} \operatorname{comp}_{L, \text { iso,rel }}(\cdot) \subset{ }^{b} \mathfrak{M}_{L, \text { iso,rel }}^{n}(m f)$.

REMARK 4.11. If $\left(M_{1}, g_{1}\right),\left(M_{2}, g_{2}\right) \in$ gen ${ }^{b} \operatorname{comp}_{L, \text { iso, rel }}(M, g)$, then, in general, $\left(M_{1}, g_{1}\right)$ $\#\left(M_{2}, g_{2}\right) \notin$ gen ${ }^{b} \operatorname{comp}_{L, \text { iso }, \text { rel }}(M, g)$.

Let $\Omega_{n}^{n c}\left(c s\right.$, gen $\left.{ }^{b} \operatorname{comp}_{L, \text { iso,rel }}(M, g)\right) \subset \Omega_{n}^{n c}(c s)$ be the subgroup generated by

$$
\left\{\left[M^{\prime}, g^{\prime}\right]_{c s} \mid\left(M^{\prime}, g^{\prime}\right) \in \text { gen }^{b} \operatorname{comp}_{L, \text { iso, rel }}(M, g)\right\} \text {. }
$$

We know $\Omega_{n}^{n c}(c s)$ completely if we know all $\Omega_{n}^{n c}\left(c s\right.$, gen $\left.{ }^{b} \operatorname{comp}_{L, \text { iso,rel }}(M, g)\right)$, and we know

$$
\Omega_{n}^{n c}\left(c s,{ }^{b} \operatorname{comp}_{L, i s o, r e l}(M, g)\right)
$$

completely if we know a corresponding generating set. However, the elements of such a set are completely determined by their (relative) characteristic numbers.

Fix $\left(M^{n}, g\right) \in$ gen ${ }^{b} \operatorname{comp}_{L, \text { iso, rel }}\left(M^{n}, g\right)$, where $M$ is oriented. Assume that $\left(M_{1}, g_{1}\right) \in$ gen ${ }^{b}$ comp $_{L, \text { iso,rel }}\left(M^{n}, g\right)$, and let $\Phi: M \backslash K \rightarrow M_{1} \backslash K_{1}$ be an orientation preserving isometry. Define the (relative) Stiefel-Whitney numbers of the pair $\left(M_{1}, M\right)$ by

$$
w_{1}^{r_{1}} \ldots w_{n}^{r_{n}}\left(M_{1}, M\right):=\left\langle w_{1}^{r_{1}} \ldots w_{n}^{r_{n}},\left[K_{1}\right]\right\rangle+\left\langle w_{1}^{r_{1}} \ldots w_{n}^{r_{n}},[K]\right\rangle .
$$

Similarly, for $\left(M_{1}, M\right)$ and $n=4 k$, we define the (relative) Pontrjagin numbers

$$
p_{1}^{r_{1}} \ldots p_{k}^{r_{k}}\left(M_{1}, M\right):=\int_{K_{1}} p_{1}^{r_{1}} \ldots p_{k}^{r_{k}}\left(M_{1}\right)-\int_{K} p_{1}^{r_{1}} \ldots p_{k}^{r_{k}}(M)
$$

and the (relative) signature by

$$
\sigma\left(M_{1}, M\right):=\sigma\left(K_{1}\right)+\sigma(-K) .
$$

LEMmA 4.12. The numbers $w_{1}^{r_{1}} \ldots w_{n}^{r_{n}}\left(M_{1}, M\right), p_{1}^{r_{1}} \ldots p_{k}^{r_{k}}\left(M_{1}, M\right)$, and $\sigma\left(M_{1}, M\right)$ are well defined, and we have

$$
\begin{aligned}
w_{1}^{r_{1}} \ldots w_{n}^{r_{n}}\left(M_{1}, M\right) & =\left\langle w_{1}^{r_{1}} \ldots w_{n}^{r_{n}}\left(K_{1} \cup K\right),\left[K_{1} \cup K\right]\right\rangle, \\
p_{1}^{r_{1}} \ldots p_{k}^{r_{k}}\left(M_{1}, M\right) & =\left\langle p_{1}^{r_{1}} \ldots p_{k}^{r_{k}}\left(K_{1} \cup-K\right),\left[K_{1} \cup-K\right]\right\rangle,
\end{aligned}
$$

and

$$
\sigma\left(M_{1}, M\right)=\sigma\left(K_{1} \cup-K\right) .
$$

Proof. The equations (4.3), (4.4) are clear. (4.5) comes from Novikov additivity of $\sigma$. Hence we have only to show the well definedness, i.e. the independence of the choice of $K \subset M, K_{1} \subset M_{1}$. Start with (4.4). If $K_{1}^{\prime} \supset K_{1}, K^{\prime} \supset K,\left.\Phi\right|_{M \backslash K^{\prime}}: M \backslash K^{\prime} \stackrel{\cong}{\longrightarrow} M_{1} \backslash K_{1}^{\prime}$ orientation preserving isometric, then

$$
\int_{K_{1}^{\prime}} \cdots-\int_{K^{\prime}} \cdots=\int_{K_{1}^{\prime} \backslash \stackrel{\mathrm{o}}{1}_{1}} \cdots+\int_{K_{1}} \cdots-\left(\int_{K^{\prime} \backslash \mathrm{o}} \cdots+\int_{K} \cdots\right) .
$$

But $\int_{K_{1}^{\prime} \backslash K_{1}} \cdots-\int_{K^{\prime} \backslash \stackrel{\circ}{K}} \cdots=0$ since $K_{1}^{\prime} \backslash \stackrel{\circ}{K_{1}}$ and $K^{\prime} \backslash \stackrel{\circ}{K}$ are isometric under $\Phi$ by assumption. The analogous conclusion can be done for $K_{1}^{\prime \prime} \subset K_{1}, K^{\prime \prime} \subset K, \Phi: M \backslash \stackrel{\circ}{K}$ " $\rightarrow$ $M_{1} \backslash \stackrel{\circ}{K_{1}^{\prime \prime}}$ already an isometry. In the general case $K_{1}, K_{1}^{\prime}, K, K^{\prime}$ one considers $K_{1}^{\prime} \cap K_{1}$, $K \cap K^{\prime}$ and reduces to the first two considerations after smoothing out $K_{1}^{\prime} \cap K_{1}, K^{\prime} \cap K$ by 
arbitrary small perturbations. The proof for (4.3) is quite similar replacing integrations in (4.9) by application of cocycles to cycles. The independence of (4.5) comes again from Novikov additivity, applying it several times.

Theorem 4.13. Fix $\left(M_{1}, g_{1}\right),\left(M_{2}, g_{2}\right) \in \operatorname{gen}{ }^{b} \operatorname{comp}_{L, i s o, r e l}(M, g)$. Then $\left(M_{1}, g_{1}\right) \underset{b, c s}{\sim}$ $\left(M_{2}, g_{2}\right)$ if and only if all characteristic numbers of $\left(M_{1}, M\right)$ coincide with the corresponding characteristic numbers of $\left(M_{2}, M\right)$.

Proof. Assume $\left(M_{1}, g_{1}\right) \underset{b, c s}{\sim}\left(M_{2}, g_{2}\right)$. Choose $C^{n+1} \subset B^{n+1}$ large enough such that with $\partial C^{n+1}=\partial_{1} C \cup \partial_{2} C \cup \partial_{3} C, \partial_{1} C=K_{1} \subset M_{1}, \partial_{2} C=K_{2} \subset M_{2}, \partial_{3} C \cong \partial K_{1} \times[0,1]$ we have $M_{1} \backslash \stackrel{\circ}{K_{1}} \underset{\Phi_{1}}{\cong} M \backslash \stackrel{\circ}{K}, M_{2} \backslash \stackrel{\circ}{\Phi_{2}} \underset{\Phi_{2}}{\cong} M \backslash \stackrel{\circ}{K}$. Then after smoothing out by arbitrary small perturbations, $\partial C$ is diffeomorphic ot $K_{1} \underset{\Phi}{\cup}-K_{2}, \Phi=\partial\left(\Phi_{2}^{-1} \Phi_{1}\right)$. Hence char.n. $\left(K_{1} \underset{\Phi}{\cup}\right.$ $\left.-K_{2}\right)=$ char.n. $\left(K_{1} \underset{\Phi_{1}}{\cup}-K\right)+$ char.n. $\left(K \underset{\Phi_{2}^{-1}}{\cup}-K_{2}\right)=$ char.n. $\left(M_{1}, M\right)+\operatorname{char} . n \cdot\left(M, M_{2}\right)=$ char.n. $\left(M_{1}, M\right)$ - char.n. $\left(M_{2}, M\right)$, i.e. char.n. $\left(M_{1}, M\right)=$ char.n. $\left(M_{2}, M\right)$. Conversely, if char.n. $\left(M_{1}, M\right)=$ char.n. $\left(M_{2}, M\right)$ then char.n. $\left(K_{1} \underset{\Phi}{\cup}-K_{2}\right)=0, K_{1} \underset{\Phi}{\cup}-K_{2}$ is 0-bordant, $K_{1} \underset{\Phi}{\cup}-K_{2}=\partial C^{n+1}$. Form $\left.K_{1} \cup\left(\partial K_{1} \times[0,1]\right) \underset{\Phi}{\cup}-K_{2}\right)$ which equals $\partial C^{n+1}$, glue $\left(M_{1} \backslash \stackrel{\circ}{K_{1}}\right)$ $\times[0,1] \cong(M \backslash \stackrel{\circ}{K}) \times[0,1] \cong\left(M_{2} \backslash \stackrel{\circ}{K}{ }_{2}\right) \times[0,1]$ and smooth out (the topology and the metrics). The result is a bordism $\left(B^{n+1}, g_{B}\right)$ with compact support between $\left(M_{1}, g\right)$ and $\left(M_{2}, g\right)$.

COROLlary 4.14. Description of all elements of $\Omega_{n}^{n c}(\mathrm{cs})$ reduces to "counting" the generalized components of $\boldsymbol{M M}_{L, \text { iso,rel }}^{n}(m f)$.

ExAmPle 4.15. Consider $M_{i}=\left(M_{i}^{\prime} \cup \partial M_{i}^{\prime} \times\left[0, \infty\left[, g_{i}\right), i=1,2, M_{i}^{\prime}\right.\right.$ compact, $\partial M_{1}^{\prime}=$ $\partial M_{2}^{\prime},\left(\partial M_{1}^{\prime} \times\left[0, \infty\left[, g_{1, a, \infty}\right)\right.\right.$ isometric to $\left(\partial M_{2}^{\prime} \times\left[0, \infty\left[, g_{2, a, \infty}\right), g_{i, a, \infty}=\left.g_{i}\right|_{\partial M_{i}^{\prime} \times[a, \infty[}\right.\right.$. Let $M=\left(D^{n} \cup S^{n} \times\left[0, \infty\left[, g_{\text {standard }}\right)\right.\right.$. If $\sigma\left(M_{1}^{\prime}\right) \neq \sigma\left(M_{2}^{\prime}\right)$ then $\left(M_{1}, g\right),\left(M_{2}, g\right) \in$ gen ${ }^{b} \operatorname{comp}_{L, \text { iso,rel }}(M, g)$ are not cs-bordant.

There is a simple approach to calculate the local algebraic structure of $\Omega_{n}^{n c}(c s)$. Consider as above $\Omega_{n}^{n c}(c s, M):=\Omega_{n}^{n c}\left(c s\right.$, gen $\left.{ }^{b} \operatorname{comp}_{L, \text { iso,rel }}(M)\right)$ and let $\Omega_{n}$ be the usual bordism group of closed oriented $n$-manifolds. Then there exists a map $\Phi=\Phi_{M}: \Omega_{n} \rightarrow$ $\Omega_{n}^{n c}(c s, M), \Phi([N]):=\left[M \# N, g_{M \# N}\right]_{c s}$. Here the bordism class in $\Omega_{n}^{n c}(c s, M)$ is independent of the metric of $N$. Moreover, we have a map $\Psi=\Psi_{M}: \Omega_{n}^{n c}(c s, M) \rightarrow \Omega_{n}$, $\Psi\left(\left[M^{\prime}, g^{\prime}\right]\right):=\left[K^{\prime} \cup-K\right]$, where $\left(M^{\prime} \backslash \stackrel{\circ}{K}{ }^{\prime},\left.g^{\prime}\right|_{M^{\prime} \backslash \stackrel{\circ}{K}}\right)$ is isometric to $\left(M \backslash \stackrel{\circ}{K},\left.g\right|_{M \backslash \stackrel{\circ}{K}}\right)$. It is very easy to see that $\Psi_{M}$ is well defined: Let $\left(M^{\prime \prime}, g^{\prime \prime}\right) \in\left[M^{\prime}, g^{\prime}\right]_{c s}$. Then there exist $K_{1}^{\prime \prime} \subset M^{\prime \prime}, K_{1}^{\prime} \subset M^{\prime}, K_{1} \subset M$ such that $M^{\prime \prime} \backslash \dot{K}_{1}^{\prime \prime}, M^{\prime} \backslash \dot{K}_{1}^{\prime}, M \backslash \dot{K}_{1}$ are isometric. By assumption and according to theorem 4.12 we have char.n. $\left(M^{\prime \prime}, M\right)=$ char.n. $\left(M^{\prime}, M\right)$, i.e. char.n. $\left(K_{1}^{\prime \prime} \cup-K_{1}\right)=\operatorname{char.n.}\left(K_{1}^{\prime} \cup-K_{1}\right)=\operatorname{char} . n .\left(K^{\prime} \cup-K\right)$, $\left[K_{1}^{\prime \prime} \cup-K_{1}\right]_{\Omega_{n}}=\left[K^{\prime} \cup-K\right]_{\Omega_{n}}$. We have $\Psi_{M} \Phi_{M}=\mathrm{id}:(\Psi \Phi)[N]=\Psi([M \# N])=[N]$ and $\Phi_{M} \Psi_{M}=\mathrm{id}:(\Phi \Psi)\left(\left[M^{\prime}, g^{\prime}\right]\right)=\Phi\left(\left[K^{\prime} \cup K\right]\right)=\left[M \#\left(K^{\prime} \cup-K\right), g_{M \#\left(K^{\prime} \cup-K\right)}\right]=\left[M^{\prime}, g^{\prime}\right]$ since char.n. $\left(M^{\prime}, M\right)=$ char.n. $\left(M \#\left(K^{\prime} \cup-K\right), M\right)$. Here $\Phi_{M}$ and $\Psi_{M}$ are 1-1 maps. Moreover we have maps 


$$
\begin{aligned}
& \Phi_{M} \times \Phi_{M^{\prime}}: \Omega_{n} \times \Omega_{n} \rightarrow \Omega_{n}^{n c}(c s, M) \times \Omega_{n}^{n c}\left(c s, M^{\prime}\right), \\
& \Phi_{M} \times \Phi_{M^{\prime}}\left(\left[N_{1}\right],\left[N_{2}\right]\right)=\left(\left[M \# N_{1}, g_{M \# N_{1}}\right],\left[M^{\prime} \# N_{2}, g_{M^{\prime} \# N_{2}}\right]\right) \text {, } \\
& \Phi_{M \# M^{\prime}}: \Omega_{n} \rightarrow \Omega_{n}^{n c}\left(c s, M \# M^{\prime}\right) \text {, }
\end{aligned}
$$

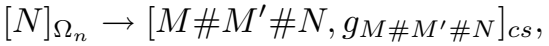

$$
\begin{aligned}
& \Omega_{n} \times \Omega_{n}, \quad\left(\left[N_{1}\right],\left[N_{2}\right]\right) \\
& \downarrow \\
& \Omega_{n} \quad\left[N_{1} \# N_{2}\right]
\end{aligned}
$$

and

$$
\begin{array}{cc}
\Omega_{n}^{n c}(c s, M) \times \Omega_{n}^{n c}\left(c s, M^{\prime}\right), & \left(\left[M_{1}, g_{1}\right],\left[M_{1}^{\prime}, g_{1}^{\prime}\right]\right) \\
\downarrow & \downarrow \\
\Omega_{n}^{n c}\left(c s, M \# M^{\prime}\right) & {\left[M_{1} \# M_{1}^{\prime}, g_{1} \# g_{1}^{\prime}\right]}
\end{array}
$$

Proposition 4.16. The diagrams

$$
\begin{array}{ccc}
\Omega_{n} \times \Omega_{n} & \stackrel{\Phi_{M} \times \Phi_{M^{\prime}}}{\longrightarrow} & \Omega_{n}^{n c}(c s, M) \times \Omega_{n}^{n c}\left(c s, M^{\prime}\right) \\
\downarrow & & \downarrow \\
\Omega_{n} & \stackrel{\Phi_{M \# M^{\prime}}}{\longrightarrow} & \Omega_{n}^{n c}\left(c s, M \# M^{\prime}\right)
\end{array}
$$

and

$$
\begin{array}{ccc}
\Omega_{n}^{n c}(c s, M) \times \Omega_{n}^{n c}\left(c s, M^{\prime}\right) & \stackrel{\Phi_{M} \times \Phi_{M^{\prime}}}{\longrightarrow} & \Omega_{n} \times \Omega_{n} \\
\downarrow & \downarrow \\
\Omega_{n}^{n c}\left(c s, M \# M^{\prime}\right) & \stackrel{\Phi_{M \# M^{\prime}}}{\longrightarrow} & \Omega_{n}
\end{array}
$$

commute.

REMARK 4.17. It is important that we consider (4.10), (4.11) at bordism class level. In (4.11) e.g. $\left(K_{1} \cup-K\right) \#\left(K_{1}^{\prime} \cup-K^{\prime}\right) \neq K_{1} \# K_{1}^{\prime} \cup-\left(K \# K^{\prime}\right)$, but their bordism classes coincide.

The 1-1 property of $\Phi_{M}, \Psi_{M}$ moreover implies

Proposition 4.18. Modulo torsion (which is well defined at the $\Omega_{n}$-level) we have $\Omega_{n}^{n c}\left(c s, M^{n}\right)=0$ for $n \neq 4 k$, for $n=4 k,\left[M \# P^{2 i_{1}}(\mathbb{C}) \times \cdots \times P^{2 i_{k}}(\mathbb{C}), g_{\left.M \# P^{2 i_{1}}(\mathbb{C}) \times \cdots \times P^{2 i_{k}(\mathbb{C})}\right]}\right]$ are independent generators for $\Omega_{n}^{n c}\left(\right.$ cs, $\left.M^{n}\right)$ over $\mathbb{Q}, i_{1}+\cdots+i_{k}=k$.

REMARKS 4.19. 1) $4.13,4.14,4.18$ provide sufficient means to characterize cs-bordism classes and to calculate $\Omega_{n}^{n c}(c s)$.

2) An analogous procedure can be applied to calculate e.g. $\Omega_{n}^{n c, s p i n}(c s, M)$, where ${ }^{b}$ comp $_{L, \text { iso,rel }}(M)$ is now a component consisting of Spin-manifolds.

As we pointed out, a contentful theory should be developed under three aspects.

1) A convenient characterization of bordism classes is desirable.

2) It should be possible to exhibit sets of independent generators, at least for the intersections with gen-components.

3) A geometric realization of zero and the inverse are desirable. 
The general bordism group $\Omega_{n}^{n c}$ did not satisfy any of these three wishes. $\Omega_{n}^{n c}(c s)$ satisfies the first two wishes. We develop below a bordism theory which satisfies the second and the third wish. This will be the bordism theory for manifolds with a finite number of ends, each of them nonexpanding.

Let $\varepsilon$ be an isolated end of $\left(M^{n}, g\right)$. A ray in $\varepsilon$ is a geodesic $\gamma$ defined on $[0, \infty[$ which is a shortest geodesic between any two of its points and such that some neighborhood of $\varepsilon$ contains up to a finite segment the whole of $|\gamma|$. Then the latter holds for any neighborhood of $\varepsilon$.

LEMMA 4.20. Let $\varepsilon$ be an isolated end of $\left(M^{n}, g\right)$.

a) Then there exists a ray in $\varepsilon$.

b) If $\left(M^{n}, g\right)$ additionally satisfies $(I)$ then there exists a ray in $\varepsilon$ with a uniformly thick neighborhood.

Proof. A proof of a) is e.g. contained in [11], p. 43. b) follows immediately from a) and $(I)$.

We call an end $\varepsilon$ of $\left(M^{n}, g\right)$ nonexpanding if there exist a ray $\gamma$ in $\varepsilon$ and an $R=$ $R_{M}>0$ and an element $G \in \varepsilon$ such that $G \subseteq U_{R}(|\gamma|)$, roughly written $\varepsilon \subseteq U_{R}(|\gamma|)$.

In the sequel we restrict to open manifolds satisfying $(I), B(\infty)$, with finitely many ends, each of them nonexpanding.

EXAMPLES 4.21. 1) Consider the sphere $S_{r}^{n-1} \subset \mathbb{R}^{n} \subset \mathbb{R}^{n+1}$ of radius $r$ and

$$
\operatorname{chc}^{n}(r):=\left(S_{r}^{n-1} \times\left[0, \infty\left[\cup D_{r}^{n}, g_{s t}\right),\right.\right.
$$

i.e. the closed half cylinder of radius $r$ with a standard metric $g_{s t}$ which should be the product metric of $S_{r}^{n-1} \times\left[0, \infty\right.$ [ smoothly extended to the glued bottom $D_{r}^{n}$ and with standard orientation. Then $\operatorname{chc}^{n}(r)$ is an open manifold with one nonexpanding end, satisfying $(I),\left(B_{\infty}\right)$.

2) $\#_{i=1}^{k} \operatorname{chc}\left(r_{i}\right)$ has finitely many nonexpanding ends.

3) Any manifold $\left(M^{n}=M^{\prime} \cup \partial M^{\prime} \times\left[0, \infty\left[, g_{M}\right)\right.\right.$ where $M^{\prime}$ is compact and $\left.g_{M}\right|_{\partial M^{\prime} \times[a, \infty[}=$ $d t^{2}+g_{\partial M^{\prime}}$ satisfying $(I),\left(B_{\infty}\right)$ and has finitely many nonexpanding ends.

4) The same is true if we allow $g_{M}$ of 3) to vary in $\operatorname{comp}^{p, r}\left(g_{M}\right) \cap C^{\infty}$.

5) If we consider $M$ of smooth type of 3) and $\left.g_{M}\right|_{\partial M^{\prime} \times[a, \infty[}=d t^{2}+f(t)^{2} g_{\partial M^{\prime}}$ with $c_{2} \geq f(t) \geq c_{1}>0, \frac{f^{(\nu)}}{f}$ bounded for all $\nu, t \geq a$ then $M$ has finitely many nonexpanding ends.

We define now a slightly sharpened bordism relation.

Let $\left(M^{n}, g\right),\left(M^{\prime n}, g^{\prime}\right)$ be as above, each with finitely many nonexpanding ends $\varepsilon_{1}, \ldots, \varepsilon_{s}$ or $\varepsilon_{1}^{\prime}, \ldots, \varepsilon_{s^{\prime}}^{\prime}$, respectively. Let $\gamma_{M, 1}, \ldots, \gamma_{M, s}$ or $\gamma_{M^{\prime}, 1}, \ldots, \gamma_{M^{\prime}, s^{\prime}}$ corresponding rays as above. From $(M, g) \underset{b g}{\sim}\left(M^{\prime}, g^{\prime}\right)$ and all ends nonexpanding follows in particular that for all sufficiently large compact $C^{n+1} \subset B^{n+1}$ there exists $R=R_{B}>0$ s.t.

$$
B^{n+1} \backslash C^{n+1} \subset \bigcup_{1}^{s} U_{R}\left(\left|\gamma_{M, \sigma}\right|\right), \quad B^{n+1} \backslash C^{n+1} \subset \bigcup_{1}^{s^{\prime}} U_{R}\left(\left|\gamma_{M^{\prime}, \sigma^{\prime}}\right|\right) .
$$


We require additionally the additive compatibility of the inner $\gamma$-distance and the $(B \backslash C)$ distance for points $x_{\gamma}, y_{\gamma}$ on the $\gamma^{\prime}$ s.

There exist $C^{n+1} \subset B^{n+1}$ and $c^{\prime}>0$ s.t. for $x_{\gamma}, y_{\gamma} \in|\gamma| \backslash C$,

$$
d_{\gamma}\left(x_{\gamma}, y_{\gamma}\right)-c^{\prime} \leq d_{B \backslash C}\left(x_{\gamma}, y_{\gamma}\right) \leq d_{\gamma}\left(x_{\gamma}, y_{\gamma}\right)+c^{\prime} .
$$

Here $\gamma$ stands for $\gamma_{M, 1}, \ldots, \gamma_{M, s}, \gamma_{M^{\prime}, 1}, \ldots, \gamma_{M^{\prime}, s^{\prime}}$, respectively and $d(\cdot, \cdot) \equiv \operatorname{dist}(\cdot, \cdot)$.

We denote $(M, g) \underset{n e}{\sim}\left(M^{\prime}, g^{\prime}\right)$ if they are bg-bordant by means of $\left(B, g_{B}\right)$ satisfying $(G H)$.

REMARKS 4.22. 1) The right hand inequality of $(G H)$ trivially holds. We added it only for symmetry reasons.

2) It was essentially Thomas Schick who pointed out to the author the meaning of the condition $(G H)$ or $\left(G H_{1}\right)$ and who proposed to include them into the definition of bordism.

We consider instead of $(G H)$ the condition:

There exist $C^{n+1} \subset B^{n+1}$ and $c^{\prime}>0$ s.t. for all $x, y \in U(\varepsilon)$,

$$
d_{U_{\varepsilon}}(x, y)-c \leq d_{B \backslash C}(x, y) \leq d_{U(\varepsilon)}(x, y)+c .
$$

Here $\varepsilon$ stands for $\varepsilon_{1}, \ldots, \varepsilon_{s}, \varepsilon_{1}^{\prime}, \ldots, \varepsilon_{s^{\prime}}^{\prime}$ and $U(\varepsilon)$ for a neighborhood of $\varepsilon, U(\varepsilon) \cap C=\emptyset$.

LEMMA 4.23. $(G H)$ and $\left(G H_{1}\right)$ are equivalent.

Proof. Assume $\left(G H_{1}\right)$. Then $(G H)$ holds since for $x_{\gamma}, y_{\gamma} \in|\gamma| \subset U(\varepsilon), U(\varepsilon) \cap C=\emptyset$, $d_{U(\varepsilon)}\left(x_{\gamma}, y_{\gamma}\right)=d_{\gamma}\left(x_{\gamma}, y_{\gamma}\right)$. If conversely $x, y \in U(\varepsilon)$ then there exist $x_{\gamma}, y_{\gamma} \in|\gamma| \subset U(\varepsilon)$ s.t. $d_{U(\varepsilon)}\left(x, x_{\gamma}\right) \leq R_{M}, d_{U(\varepsilon)}\left(y, y_{\gamma}\right) \leq R_{M}$. Then the assertion follows from

$$
\begin{aligned}
& d_{U(\varepsilon)}(x, y)-d_{U(\varepsilon)}\left(x_{\gamma}, y_{\gamma}\right) \leq d_{U(\varepsilon)}\left(x, x_{\gamma}\right)+d_{U(\varepsilon)}\left(y, y_{\gamma}\right), \\
& d_{U(\varepsilon)}(x, y)-d_{U(\varepsilon)}\left(x, x_{\gamma}\right)-d_{U(\varepsilon)}\left(y, y_{\gamma}\right) \leq d_{\gamma}\left(x_{\gamma}, y_{\gamma}\right) \\
& =d_{\gamma}\left(x_{\gamma}, y_{\gamma}\right)-c^{\prime}+c^{\prime} \leq d_{B \backslash C}\left(x_{\gamma}, y_{\gamma}\right)+c^{\prime} \\
& d_{U(\varepsilon)}-2 R_{M}-c^{\prime} \leq d_{B \backslash C}\left(x_{\gamma}, y_{\gamma}\right) \\
& d_{U(\varepsilon)}-4 R_{M}-c^{\prime} \leq d_{B \backslash C}(x, y) .
\end{aligned}
$$

REMARK 4.24. $\left(G H_{1}\right)$ immediately implies that $d_{G H}(\overline{B \backslash C}, \overline{U(\varepsilon)})<\infty$, where $d_{G, H}(\cdot, \cdot)$ is the Gromov-Hausdorff distance between proper metric spaces. This follows from the following facts. $d_{G H}(\overline{B \backslash C}, \overline{U(\varepsilon)})<\infty$ if we endow $\overline{U(\varepsilon)}$ with the induced length metric and use $\overline{B \backslash C} \subset U_{R}(U(\varepsilon))$. Then we use $d_{G H}(U(\varepsilon))$, its own length metric, $U(\varepsilon)$, induced length metric $<\infty$, which follows from $\left(G H_{1}\right)$. As a matter of fact, we introduced $(G H)$ to assure $d_{G H}(\overline{B \backslash C}, \overline{U(\varepsilon)})<\infty$.

PROPOSITION 4.25. $\underset{n e}{\sim}$ is an equivalence relation.

Proof. Reflexivity $\left(\left(B=m \times[0,1], d t^{2}+g_{M}\right)\right)$ and symmetry $\left(\left(-B, g_{B}\right)\right)$ are immediately clear. For transitivity, the only point in the proof is $(G H)$ or $\left(G H_{1}\right)$. Let $\left(B_{12}, g_{12}\right)$ and $\left(B_{23}, g_{23}\right)$ be fe, ne-bordisms between $\left(M_{1}, g_{1}\right),\left(M_{2}, g_{2}\right)$ and $\left(M_{2}, g_{2}\right),\left(M_{3}, g_{3}\right)$, respectively and set $\left(B_{13}, g_{13}\right)=\left(B_{12} \cup B_{23}, g_{12} \cup g_{23}\right)$. We assume w.l.o.g. global Riemannian collars at the boundaries. Let $C_{12}^{n+1} \subset B_{12}, C_{23} \subset B_{23}$ as required in $\left(G H_{1}\right)$ and choose 
$C=C_{13}^{n+1} \supset C_{12} \cup C_{23}$. Let $\varepsilon$ be one of the ends of $M_{1}, U(\varepsilon) \subset M_{1}$. Then we have to show

$$
d_{U(\varepsilon)}(x, y)-c \leq d_{B_{13} \backslash C}(x, y) .
$$

We write in the sequel $d_{i}=d_{M_{i} \backslash C}, i=1,2, d_{12}=d_{B_{12} \backslash C}, d_{23}=d_{B_{23} \backslash C}, d_{13}=d_{B_{13} \backslash C}$.

The required $c$ in (4.12) exists for all pairs $x, y \in M_{1} \backslash C$ s.t. $d_{1}(x, y)$ can be realized by a curve in $B_{12} \backslash C$. Let $x, y \in M_{1} \backslash C$ be pair which does not have this property and let $z(t)$ be a curve in $B_{13} \backslash C$ which realizes $d_{13}(x, y), z(0)=x=\alpha_{1}, z(1)=y \equiv y_{1}$. Then there exists a first point $x_{2} \in M_{2} \backslash C$ on $\left\{z_{t}\right\}_{t}$ and a last point $y_{2} \in M_{2} \backslash C$ on $z$.

Let moreover $x_{2}^{\prime} \in M_{2} \backslash C$ and $y_{2}^{\prime} \in M_{2} \backslash C$ be points which realize the distances $d_{13}\left(x_{1}, M_{2} \backslash \stackrel{\circ}{C}\right)$ and $d_{13}\left(y_{1}, M_{2} \backslash \stackrel{\circ}{C}\right)$, respectively. Then

$$
\begin{aligned}
& d_{13}\left(x_{2}^{\prime}, y_{2}^{\prime}\right)-d_{13}\left(x_{1}, y_{1}\right) \leq d_{13}\left(x_{1}, x_{2}^{\prime}\right)+d_{13}\left(y_{1}, y_{2}^{\prime}\right) \\
& d_{13}\left(x_{2}^{\prime}, y_{2}^{\prime}\right)-2 R_{12} \leq d_{13}\left(x_{1}, y_{1}\right)
\end{aligned}
$$

Claim. There exists a $c^{\prime}>0$ s.t.

$$
d_{2}\left(x_{2}^{\prime}, y_{2}^{\prime}\right)-c^{\prime} \leq d_{13}\left(x_{2}^{\prime}, y_{2}^{\prime}\right)
$$

for all $x_{2}^{\prime}, y_{2}^{\prime} \in M_{2} \backslash C$.

LEMMA 4.26. ( $C l)$ implies (4.12).

Proof. We infer from (4.13) and $(\mathrm{Cl})$

$$
d_{2}\left(x_{2}^{\prime}, y_{2}^{\prime}\right)-c^{\prime}-2 R_{12} \leq d_{13}\left(x_{1}, y_{1}\right) \text {. }
$$

Moreover

$$
\begin{aligned}
& d_{12}\left(x_{2}^{\prime}, y_{2}^{\prime}\right) \leq d_{2}\left(x_{2}^{\prime}, y_{2}^{\prime}\right), \\
& d_{12}\left(x_{1}, y_{1}\right)-2 R_{12} \leq d_{12}\left(x_{2}^{\prime}, y_{2}^{\prime}\right), \\
& d_{1}\left(x_{1}, y_{1}\right)-c_{12} \leq d_{12}\left(x_{1}, y_{1}\right) .
\end{aligned}
$$

Here (4.17) is the condition $\left(G H_{1}\right)$ for $B_{12}$. (4.13)-(4.17) yield $d_{13}\left(x_{1}, y_{1}\right) \geq d_{2}\left(x_{2}^{\prime}, y_{2}^{\prime}\right)-$ $c^{\prime}-2 R_{12} \geq d_{12}\left(x_{2}^{\prime}, y_{2}^{\prime}\right)-c^{\prime}-2 R_{12} \geq d_{12}\left(x_{1}, x_{2}\right)-4 R_{12}-c^{\prime} \geq d_{1}\left(x_{1}, y_{1}\right)-4 R_{12}-c^{\prime}-c_{12}$, i.e. we obtain (4.12) with $c=4 R_{12}+c^{\prime}+c_{12}$.

There remains to establish $(C l)$, i.e.

$$
d_{2}\left(x_{2}^{\prime}, y_{2}^{\prime}\right)-c^{\prime} \leq d_{13}\left(x_{2}^{\prime}, y_{2}^{\prime}\right) .
$$

Unfortunately $(\mathrm{Cl})$ is wrong and hence the whole approach to prove transitivity. Uwe Abresch has constructed an explicit ingenious counterexample. Nevertheless, we performed the proof of transitivity until $(\mathrm{Cl})$ to indicate the crucial point. It is in fact possible that an appropriate winding around the middle $M_{2}, M_{2}$ again and again penetrating curve has a much shorter $B_{13}$-length than the $M_{2}$-distance of the initial and the other intersection points in $M_{2}$. From this it is clear that one should forbid such a $B_{13}$-distance diminishing curve. But this can be achieved by forbidding a distance realizing curve to move out from the collar. That is if $x, y \in U(\varepsilon) \subset M_{1}$ or $M_{3}$ (or $x, y \in$ the corresponding geodesic ray, which is equivalent) then a $B_{13}$-distance realizing curve should remain in the collar of $M_{1}$ or $M_{3}$, respectively. For this we perform a conformal change in the metric $g_{12} \cup g_{23}$ of $B_{13}$. 
We consider in $U_{\delta}\left(\partial B_{13}\right)$ Gaussian normal coordinates which give for boundary points $\in \partial B_{13}$ coordinates only for a half ball. Nevertheless we have still about $x_{0} \in U_{\delta}\left(\partial B_{13}\right)$

$$
a_{13}^{\partial B}\left(x_{0}\right)|\xi|^{2} \leq g_{13, i j}(x) \xi^{i} \xi^{j} \leq b_{13}^{\partial B}\left(x_{0}\right)|\xi|^{2}, \quad \operatorname{dist}\left(x_{0}, \partial B\right) \leq \operatorname{dist}\left(x, x_{0}\right) \leq \delta / 2
$$

and about $x_{0} \in B_{13} \backslash U_{\frac{3}{4} \delta}\left(\partial B_{13}\right)$

$$
a_{13}^{\partial B}\left(x_{0}\right)|\xi|^{2} \leq g_{13, i j}(x) \xi^{i} \xi^{j} \leq b_{13}^{\partial B}\left(x_{0}\right)|\xi|^{2}, \quad \operatorname{dist}\left(x_{0}, x\right) \leq \delta / 2 .
$$

Set $a_{13}^{\partial B}=\sup _{x_{0} \in U_{\delta}(\partial B)} a_{13}^{\partial B}\left(x_{0}\right), b_{13}^{\partial B}=\inf _{x_{0} \in U_{\delta}(\partial B)} b_{13}^{\partial B}\left(x_{0}\right), a_{13}^{B}=\sup _{x_{0} \text { as above }} a_{13}^{B}\left(x_{0}\right)$, $b_{13}^{B}=\inf _{x_{0} \text { as above }} b_{13}^{B}\left(x_{0}\right)$. All these numbers are $>0$. Then we have for $x \in U_{\delta}\left(\partial B_{13}\right)$, $y \in B_{13} \backslash U_{\frac{3}{4} \delta}\left(\partial B_{13}\right)$ and $0 \neq \xi \in \mathbb{R}^{n+1}$

$$
\begin{aligned}
& \qquad g_{13, i j}(x) \xi^{i} \xi^{j} \leq b_{13}^{\partial B} \leq b_{13}^{\partial B}|\xi|^{2}=\frac{b_{13}^{\partial B}}{a_{13}^{\partial B}} a_{13}^{B}|\xi|^{2} \leq \frac{b_{13}^{\partial B}}{a_{13}^{\partial B}} g_{13, i j}(y) \xi^{i} \xi^{j} \\
& \text { Define now } \varphi \in C^{\infty}\left(B_{13}\right) \text { as follows: } \quad<\left(\frac{b_{13}^{\partial B}}{a_{13}^{\partial B}}+1\right) g_{13, i j}(y) \xi^{i} \xi^{j} .
\end{aligned}
$$

$$
\begin{aligned}
& \varphi=1 \text { on } U_{\frac{1}{2} \delta}(\partial B) . \\
& \varphi=\left(\frac{b_{13}^{\partial B}}{a_{13}^{B}}+1\right) \text { on } B_{13} \backslash U_{\frac{3}{4} \delta}(\partial B) .
\end{aligned}
$$

$\varphi$ is increasing in inward normal direction in $U_{\delta}\left(M_{1}\right)$ and decreasing in outward normal direction in $U_{\delta}\left(M_{3}\right)$,

$$
\left|\nabla^{i} \varphi\right| \leq D_{i}, \quad i=0,1,2, \ldots
$$

The existence of such a $\varphi$ in the case of bounded geometry is standard (cf. [1]).

LEMMA 4.27. a) $\tilde{g}_{13}=\varphi \cdot g_{13}$ is a metric of bounded geometry.

b) $\left(B_{12}, \tilde{g}_{13}\right)$ is a bordism between $\left(M_{1}, g_{1}\right)$ and $\left(M_{3}, g_{3}\right)$ in the bounded geometry nonexpanding sense.

Proof. a) Inside the $\frac{\delta}{2}$-collar nothing changes. $g$ and $g^{\prime}$ are quasi isometric. Moreover for $i \geq 1\left|\nabla^{i}(\tilde{g}-g)\right|=\left|\left(\nabla^{i} \varphi \cdot g\right)\right| \leq c_{i}$ for all $i$, i.e. $\tilde{g} \in{ }^{b, \infty} \operatorname{comp}(g)$. The assertion now immediately follows.

b) By assumption $B_{12} \subset U_{R_{12}}\left(M_{i}\right)=\bigcup_{x \in M_{i}} U_{R_{12}}(x), i=1,2, B_{23} \subset U_{R_{23}}\left(M_{i}\right)=$ $\bigcup_{x \in M_{i}} U_{R_{23}}(x), i=2,3$, hence $B_{13}=B_{12} \cup B_{23} \subset \bigcup_{x \in M_{i}} U_{R_{12}+R_{23}}(x)=U_{R_{13}}\left(M_{i}\right)$, $i=1,3 . g$ and $g^{\prime}$ are quasi isometric, hence $B_{13} \subset U_{\tilde{R}_{13}}\left(M_{i}\right), i=1,3$. $\left(B_{13}, \tilde{g}_{13}\right)$ satisfies $(G H)$ since for $x_{\gamma}, y_{\gamma} \in|\gamma| \backslash C$ in $M_{i}$ no distance realizing or approximating curve in $B_{13}$ will leave $U_{\frac{1}{2} \delta}\left(M_{i}\right), i=1$ or 3 . This follows from indroducing normal charts and applying (4.18). But in the collar $U_{\frac{1}{2} \delta}\left(M_{i}\right),(G H)$ holds. According to the Pythagoraen principle the curve remains in $M_{i}$, it is even $\gamma$. This finishes the proof of proposition 4.25.

$\Omega_{n}^{n c}(n e) \equiv \Omega^{n c}\left(f_{e}, n e, b_{g}\right)$ is again defined as Grothendieck group. Next we develop geometric realizations for 0 and $-[M, g]_{n e}$ in $\Omega_{n}^{n c}(n e)$.

Let $\left(M^{n}, g\right)$ be as above, i.e. oriented, with $(I),\left(B_{\infty}\right)$, finitely many ends $\varepsilon_{1}, \ldots, \varepsilon_{s}$, each of them nonexpanding. Let $\varepsilon$ be one of them, $C \subset M$ compact and so large that $\varepsilon$ is defined by one of the components of $M \backslash C, U_{\varepsilon} \subset M \backslash C$ a neighborhood, $\gamma$ a ray in $U(\varepsilon)$. $\gamma$ admits a tubular neighborhood of radius $\delta_{3}>0$. Consider $\left(B, g_{B}\right)=\left(M \times I, g_{M}+d r^{2}\right)$. Then $\varepsilon \times I=\left\{U_{j}(\varepsilon) \times I\right\}_{j \in J}$ is an end of $M \times I, U(\varepsilon \times I)=U(\varepsilon) \times I$ a neighborhood 
disjoint from $C_{M \times I}=C \times I$, and for $0<\delta_{1}<1$, the curve $\gamma_{\delta_{1}}=\gamma \times\left\{\delta_{1}\right\}=\left(\gamma, \delta_{1}\right)$ is a ray in $U(\varepsilon \times I) . \varepsilon \times I$ is nonexpanding. $\gamma_{\delta_{1}}$ admits a tubular neighborhood with a radius $\delta_{2}>0, T_{\delta_{2}}\left(\gamma_{\delta_{1}}\right)$.

THEOREM 4.28. $\partial T_{\delta_{2}}\left(\gamma_{\delta_{1}}\right)$ has bounded geometry, one nonexpanding end and

$$
\partial T_{\delta_{2}}\left(\gamma_{\delta_{1}}\right) \underset{n e}{\sim} \operatorname{chc}^{n}\left(\delta_{2}\right), \quad \delta_{2}>0 .
$$

Proof. First we show that $\partial T=\partial T_{\delta_{2}}\left(\gamma_{\delta_{1}}\right)$ has bounded geometry. Consider the point $x=\exp _{\gamma_{\delta_{1}}(t)}\left(\delta_{1} \cdot u\right), \delta_{1} u \perp \dot{\gamma}_{\delta_{1}}(t), x \in \partial T$, and the equation $B_{U}^{\prime \prime}+R^{M \times I} B_{U}=0$ along $\sigma(\tau)=\exp _{\gamma_{\delta_{1}}(t)} \tau\left(\delta_{1} u\right)$ for the endomorphism valued function $B_{U}: \tau \rightarrow B_{U}(\tau)$, $B_{U}(\tau):(\dot{\gamma})^{\perp} \rightarrow(\dot{\gamma})^{\perp}$, with the initial conditions $B_{U}(0)=\left(\begin{array}{ll}1 & 0 \\ 0 & 0\end{array}\right), B_{U}^{\prime}(0)=\left(\begin{array}{lll}0 & 0 \\ 0 & I_{n-1}\end{array}\right)$. Then $\left|\nabla^{i} B_{U}\right|\left(\delta_{1}\right) \leq C_{i}, C_{i}$ independent of $u$ and $t$ since the curvature tensor $R^{M \times I}$ satisfies $\left(B_{\infty}\right)$. According to [24], p. 57, the second fundamental form $S_{\gamma_{\delta_{1}}}(x)$ at $x=$ $\exp _{\gamma_{\delta_{1}}(t)}\left(\delta_{1} u\right)$ is given by

$$
S_{\delta_{1}}(x)=\left(B_{U}^{\prime} B_{U}^{-1}\right)\left(\delta_{1}\right)
$$

Hence $S_{\delta_{1}}$ satisfies $\left(B_{\infty}\right)$ since $B$ does. According to Gauss' equations, $g_{\partial T}=\left.g_{M \times I}\right|_{\partial T}$ satisfies $\left(B_{\infty}\right)$. Similarly it follows that $\partial T$ satisfies $(I)$ since $\gamma, \gamma_{\delta_{1}}$ and the fibres have bounded from below diameter. We omit the trivial considerations at the bottom. $\left(\partial T, g_{\partial T}\right)$ has one end and this end is nonexpanding: $\partial T=\bigcup_{t} \Sigma_{t}$, where $\Sigma_{t}$ is the geodesic $\delta_{2}$-sphere in $M \times I$ about $\gamma_{\delta_{1}}(t)$. Each such sphere intersects $\gamma_{\delta_{1}+\delta_{2}}=\left(\gamma, \delta_{1}+\delta_{2}\right)$ once and there is a common constant $K_{\Sigma}$ which uniformly bounds the circumference of all such geodesic spheres. The latter comes from Rauch's comparison theorem. Hence $\partial T \subseteq U_{\frac{K_{\Sigma}}{2}}\left(\left|\gamma_{\delta_{1}+\delta_{2}}\right|\right)$. Next we construct a bibounded diffeomorphism from $\operatorname{chc}^{n}\left(\delta_{2}\right)$ onto $\partial T$.

Consider in $\mathbb{R}^{n+1}$ the standard basis $e_{1}, \ldots, e_{n+1}$, the geodesic $t \cdot e_{n+1}$, the parallel translation of $e_{1}, \ldots, e_{n}$ along $t \cdot e_{n+1}$, and the map $\left(x_{1}, \ldots, x_{n}\right), x_{1}^{2}+\cdots+x_{n}^{2}=\delta_{2} \rightarrow$ $x=\exp _{t e_{n+1}}\left(x_{1} e_{1}+\cdots+x_{n} e_{n}\right)$. Do the same in $M \times I$ : Let $E_{1}, \ldots, E_{n+1}$ be orthonormal at the beginning of $\gamma_{\delta_{1}}, E_{n+1}=\dot{\gamma}_{\delta_{1}}, E_{1}=\partial_{t}=\frac{\partial}{\partial t}$, translate this parallel along $\gamma_{\delta_{1}}$, and consider the map $\left(y_{1}, \ldots, y_{n}\right), y_{1}^{2}+\cdots+y_{n}^{2}=\delta_{2} \rightarrow y=\exp _{\gamma_{\delta_{1}}(t)}\left(y_{1} E_{1}+\cdots+y_{n} E_{n}\right)$. Then we get a diffeomorphism $\Phi=\exp _{\gamma_{\delta_{1}(t)}} \circ \Psi \circ \exp _{t e_{n+1}}^{-1}$ from chc ${ }^{n}\left(\delta_{2}\right)$ onto $\partial T$, where $\Psi\left(x_{1} e_{1}+\cdots+x_{n} e_{n}\right)=x_{1} E_{1}+\cdots+x_{n} E_{n}$. We omit the very simple considerations at the bottoms, respectively. According to [7], the exponential maps are $C^{\infty}$-bibounded, $\Psi$ too, hence $\Phi$, and $\Phi^{*} g_{\partial T}$ is a second metric of bounded geometry on $\operatorname{chc}^{n}\left(\delta_{2}\right)$. $\left(\partial T, g_{\partial T}\right)$ and $\left(\operatorname{chc}^{n}\left(\delta_{2}\right), \Phi^{*} g_{\partial T}\right)$ are isometric hence ne- (and bg-) bordant. Finally we want to show that $\left(\operatorname{chc}^{n}\left(\delta_{2}\right), \Phi^{*} g_{\partial T}\right)$ and $\left(\operatorname{chc}^{n}\left(\delta_{2}\right), g_{\text {standard }}\right)$ are ne-bordant. We perform this in two steps. First we show that they are bg-bordant and thereafter we verify after conformal change the conditions $\left(G H_{1}\right) . \Phi^{*} g_{\partial T}$ and $g_{s t}$ live in the same ${ }^{b, \infty}$ component: For $X \in T \operatorname{chc}^{n}\left(\delta_{2}\right)$ we have

$$
\begin{aligned}
& |X|_{\Phi^{*} g_{\partial T}}=\left|\Phi_{*} X\right|_{g \partial T} \leq\left|\Phi_{*}\right||X|_{g_{s t}}, \\
& |X|_{g_{s t}}=\left|\Phi_{*}^{-1} \Phi_{*} X\right|_{g_{s t}} \leq\left|\Phi_{*}^{-1}\right|\left|\Phi_{*} X\right|_{g \partial T}=\left|\Phi_{*}^{-1}\right||X|_{\Phi^{*} g_{\partial T}},
\end{aligned}
$$

i.e. $\Phi^{*} g_{\partial T}$ and $g_{s t}$ are quasi isometric. There remains to show that

$$
\mid\left(\nabla^{g_{s t}}\right)^{i}\left(\Phi^{*} g_{\partial T}-g_{s t}|=|\left(\nabla^{g_{s t}}\right)^{i}\left(\Phi^{*} g_{\partial T}\right) \mid\right.
$$


remains bounded for all $i$. Here we use that the (Fermi) coordinates of $x \in D_{\delta_{2}}^{n} \times[0, \infty$ [ and of $\Phi(x) \in T_{\delta_{2}}\left(\gamma_{\delta_{1}}\right)$ are the same, where we extended $\Phi$ to the full tubes. But then (4.19) follows.

According to theorem 4.28, $\left(\operatorname{chc}^{n}\left(\delta_{2}\right), \Phi^{*} g_{\partial T}\right)$ and $\left(\operatorname{chc}^{n}\left(\delta_{2}\right), g_{s t}\right)$ are bg-bordant. Let $\left(B^{n+1}, g_{B}\right)$ be a bg-bordism as in the proof of 4.28 , i.e. $\left(B^{n+1}, g_{B}\right)=\left(\operatorname{chc}^{n}\left(\delta_{2}\right) \times\right.$ $\left.[0,1], \varphi(t)+d t^{2}\right), \varphi(t)=\Phi^{*} g_{\partial T}, 0 \leq t \leq \delta, \varphi(t)=g_{s t}, 1-\delta \leq t \leq 1$, and $\varphi(t)=$ smooth convex combination of $\Phi^{*} g_{\partial T}$ and $g_{s t}$ for $\delta \leq t \leq 1-\delta$ ( $\operatorname{smoothed}$ out at $\left.\delta, 1-\delta\right)$. There remains to establish $(G H)$ or $\left(G H_{1}\right)$. We prove $(G H)$ and change for this the metric in $B^{n+1}=\operatorname{chc}^{n}\left(\delta_{2}\right) \times[0,1]$. Choose smooth functions $\psi_{1}, \psi_{2}:[0,1] \rightarrow[0,1], \psi_{1}=1$, $0 \leq t \leq 1-\frac{3}{4} \delta$, decreasing on $\left[1-\frac{3}{4} \delta, 1-\frac{1}{2} \delta\right],=0$ on $\left[1-\frac{1}{2} \delta, 1\right], \psi_{2}=0,0 \leq t \leq \frac{1}{2} \delta$, increasing on $\left[\frac{1}{2} \delta, \frac{3}{4} \delta\right],=1$ on $\left[\frac{3}{4} \delta, 1\right]$, and set our new $g_{B}=\psi_{1} \Phi^{*} g_{\partial T}+\psi_{2} g_{s t}+d t^{2}$. Then again $\left(B, g_{B}\right)$ is a bg-bordism between $\left(\operatorname{chc}^{n}\left(\delta_{2}\right), \Phi^{*} g_{\partial T}\right)$ and $\left(\operatorname{chc}^{n}, g_{s t}\right)$. We recall that if $\gamma$ is a ray in $\left(M^{n}, g_{M}\right)$ then $\gamma_{\lambda}=(\gamma, \lambda)$ is a ray in $\left(M \times[0,1], g_{M}+d t^{2}\right)$, in particular $\gamma_{\delta_{1}+\delta_{2}} \subset \partial T_{\delta_{2}}\left(\gamma_{\delta_{1}}\right)$ is a ray in $\left(M \times[0,1], g_{M}+d t^{2}\right)$ and hence in $\partial T_{\delta_{2}}\left(\gamma_{\delta_{1}}\right)$. This can easily be proven by distinct methods. Hence $\Phi^{-1} \gamma_{\delta_{1}+\delta_{2}}\left(\delta_{2}, 0, \ldots, 0, \tau\right)$ is a ray in $\left(\operatorname{chc}^{n}\left(\delta_{2}\right), \Phi^{*} g_{\partial T}\right)$. Let $x=\left(\delta_{2}, 0, \ldots, 0, \tau_{1}\right), y=\left(\delta_{2}, 0, \ldots, 0, \tau_{2}\right)$ be $\in\left(\operatorname{chc}^{n}\left(\delta_{2}\right), \Phi^{*} g_{\partial T}\right)$ and $\{c(s)\}_{0 \leq s \leq \sigma}$ be a curve in $\left(B, g_{B}\right)$ connecting $x$ and $y$. The tangent vector $\dot{c}(s)$ of $\dot{c}(s)=\left(x_{\Sigma}(s), t(s)\right)$ decomposes as orthogonal sum $\dot{c}(s)=\dot{x}_{\Sigma}(s), \dot{t}(s)=\dot{t}(s)^{\perp}+\dot{t}(s)$ and we obtain

$$
\begin{aligned}
& \text { length }(c(s))=\int_{0}^{\sigma_{2}}\left|\dot{t}(s)^{\perp}\right|_{g_{B}} d s+\int_{0}^{\sigma_{2}}|\dot{t}(s)|_{d t^{2}} d s \\
& \geq \int_{0}^{\sigma_{2}}\left|\dot{t}(s)^{\perp}\right|_{g_{B}} d s=\int_{0}^{\sigma_{2}}\left|\dot{t}(s)^{\perp}\right|_{\Psi_{1} \Phi^{*} g_{\partial T}+\Psi_{2} g_{s t}} d s .
\end{aligned}
$$

We consider several cases. The first case is that $c(s)$ remains in $\operatorname{chc}^{n}\left(\delta_{2}\right) \times\left[0,1-\frac{3}{4} \delta\right]$ which implies

$$
\left|\dot{t}(s)^{\perp}\right|_{\psi_{1} \Phi^{*} g_{\partial T}+\psi_{2} g_{s t}}=\left|\dot{t}(s)^{\perp}\right|_{\Phi^{*} g_{\partial T}} .
$$

But $x_{\Sigma}(s), \dot{x}_{\Sigma}(s)$, is a curve in $\left(\operatorname{chc}^{n}\left(\delta_{2}\right), \Phi^{*} g_{\partial T}\right)$ and hence

$$
\operatorname{length}\left(x_{\Sigma}(s)\right)=\int_{0}^{\sigma_{2}}\left|\dot{t}(s)^{\perp}\right|_{\Phi^{*} g_{\partial T}} d s \geq\left|\tau_{1}-\tau_{2}\right|
$$

according to the ray property above. Altogether, in this case

$$
\operatorname{length}(c(s)) \geq\left|\tau_{1}-\tau_{2}\right|=d_{\left(\operatorname{chc}^{n}\left(\delta_{2}\right) \cdot \Phi^{*} g_{\partial T}\right)}(x, y) .
$$

The second case under consideration is the case that at $s=s_{1}, c(s)$ leaves $\operatorname{chc}^{n}\left(\delta_{2}\right) \times$ $\left[0,1-\frac{3}{4} \delta\right]$, returns to it at $s=s_{2}$ and then remains in $\operatorname{chc}^{n}\left(\delta_{2}\right) \times\left[0,1-\frac{3}{4} \delta\right]$. In this case

$$
\begin{aligned}
& \int_{0}^{\sigma_{2}}\left|\dot{t}(s)^{\perp}\right|_{g_{B}} d s=\int_{0}^{s_{1}}\left|\dot{t}(s)^{\perp}\right|_{g_{B}} d s+\int_{s_{1}}^{s_{2}}\left|\dot{t}(s)^{\perp}\right|_{g_{B}} d s+\int_{s_{2}}^{\sigma_{2}}\left|\dot{t}(s)^{\perp}\right|_{g_{B}} d s \\
& \geq \int_{0}^{s_{1}}\left|\dot{t}(s)^{\perp}\right|_{\Phi^{*} g_{\partial T}} d s+\int_{s_{2}}^{\sigma_{2}}\left|\dot{t}(s)^{\perp}\right|_{\Phi^{*} g_{\partial T}} d s+\int_{s_{1}}^{s_{2}}\left|\dot{t}(s)^{\perp}\right|_{g_{s t}} d s .
\end{aligned}
$$


Let $x_{\Sigma}\left(s_{1}\right)=\left(\delta_{2}, 0, \ldots, 0, \tau_{11}\right), x_{\Sigma}\left(s_{1}\right)=\left(\delta_{2}, 0, \ldots, 0, \tau_{22}\right)$. Then

$$
\begin{aligned}
& \int_{0}^{s_{1}}\left|\dot{t}(s)^{\perp}\right|_{\Phi^{*} g_{\partial T}} d s \geq\left|\tau_{1}-\tau_{11}\right|, \\
& \int_{s_{2}}^{\sigma_{2}}\left|\dot{t}(s)^{\perp}\right|_{\Phi^{*} g_{\partial T}} d s \geq\left|\tau_{2}-\tau_{22}\right|, \\
& \int_{s_{1}}^{s_{2}}\left|\dot{t}(s)^{\perp}\right|_{g_{s t}} d s \geq\left|\tau_{11}-\tau_{22}\right|,
\end{aligned}
$$

which immediately implies

$$
\operatorname{length}(c(s)) \geq \int_{0}^{\delta_{2}}\left|\dot{t}(s)^{\perp}\right|_{g_{B}} d s \geq\left|\tau_{1}-\tau_{2}\right| .
$$

The most general case would be that $c(s)$ oscillates between $\left(\operatorname{chc}^{n}\left(\delta_{2}\right),\left[0,1-\frac{3}{4} \delta\right]\right)$ and its complement. In this case we have many times inequalities of type (4.25) - (4.27), sum up, apply the triangle inequality and end again at (4.28). Altogether for $x, y \in \Phi^{-1} \gamma_{\delta_{1}+\delta_{2}}$,

$$
d_{\left(B, g_{B}\right)}(x, y) \geq d_{\left(\operatorname{chc}^{n}\left(\delta_{2}\right), \Phi^{*} g_{\partial T}\right)}(x, y) .
$$

If $x, y \in \gamma_{\delta_{2}} \subset\left(\operatorname{chc}^{n}\left(\delta_{2}\right), g_{s t}\right)$ then we replace in $(4.20)-(4.28) \Phi^{*} g_{\partial T}$ by $g_{s t}$ and calculate as above,

$$
d_{\left(B, g_{B}\right)}(x, y) \geq d_{\left(\operatorname{chc}^{n}\left(\delta_{2}\right), g_{s t}\right)}(x, y) .
$$

$(G H)$ is established.

We shall see $\left(\operatorname{chc}^{n}(\delta), g_{s t}\right)$ will play the role of our zero in $\Omega_{n}^{n c}(n e)$.

LEMMA 4.29 .

$$
\begin{aligned}
& \text { a) For } r_{1}<r_{2} \text { is } \operatorname{chc}^{n}\left(r_{1}\right) \underset{n e}{\sim} \operatorname{chc}^{n}\left(r_{2}\right) . \\
& \text { b) }\left[\underset{i=1}{\#} \operatorname{chc}^{n}\left(r_{i}\right)\right]_{n e}=\left[\operatorname{chc}^{n}(r)\right]_{n e} \text { for } r>r_{1}+\cdots+r_{k} .
\end{aligned}
$$

Proof. a) is immediately clear (or follows from b)). Set for b) $r=r_{1}+\cdots+r_{k}+$ $\delta$, place $\operatorname{chc}^{n}\left(r_{1}\right) \cup \cdots \cup \operatorname{chc}^{n}\left(r_{k}\right)$ all with parallel $\left[0, \infty\left[\operatorname{direction}\right.\right.$ into $\operatorname{int}\left(\operatorname{chc}^{n}(r)\right)$, where $\operatorname{int}\left(\operatorname{chc}^{n}(r)\right)$ corresponds to $\left.\stackrel{\circ}{D}_{r}^{n} \times\right] 0, \infty\left[\right.$. Then $\operatorname{CL}\left(\operatorname{int}\left(\operatorname{chc}^{n}(r)\right) \backslash \operatorname{int}\left(\operatorname{chc}^{n}\left(r_{1}\right) \cup \cdots \cup\right.\right.$ $\left.\left.\operatorname{chc}^{n}\left(r_{k}\right)\right)\right)$ defines the desired ne-bordism.

THEOREM 4.30. For any oriented manifold $\left(M^{n}, g\right)$ of bounded geometry and a finite number of ends, each of them nonexpanding, we have

$$
\left[M^{n}, g\right]_{n e}=\left[\left(M^{n}, g\right) \cup\left(\operatorname{chc}^{n}(r), g_{s t}\right)\right] .
$$

Proof. We must construct a ne-bordism between $\left(M^{n}, g\right)$ and $-\left(\left(M^{n}, g\right) \cup\left(\operatorname{chc}^{n}(r), g_{s t}\right)\right)$. Let $\left(B^{n+1}, g_{B}\right)=\left(M \times[0,1], g_{M}+d t^{2}\right), \varepsilon$ be an end of $M, \gamma$ a ray in $\varepsilon$, form $\gamma_{\delta_{1}}=$ $\left(\gamma, \delta_{1}\right) \subset M \times[0,1], T_{\delta_{2}}\left(\gamma_{\delta_{1}}\right), \delta_{2}<\inf \left\{\frac{\delta_{1}}{2}, r_{\mathrm{inj}}(M) / 2\right\}$ and set $B_{\gamma}=B^{n+1} \backslash \operatorname{int} T_{\delta_{2}}\left(\gamma_{\delta_{1}}\right)$ with the induced metric. From our assumption $r_{\text {inj }}>0$ it follows easily that $\partial T_{\delta_{2}}\left(\gamma_{\delta_{1}}\right)$ has a smooth collar $U_{\delta}(\partial T)$. Endow $U_{\frac{\delta}{2}}$ with the product metric $g_{\frac{\delta}{2}}$ and form on $U_{\delta}-U_{\frac{\delta}{2}}$ the smooth bg-convex combination of $g_{\frac{\delta}{2}}$ and $g_{B}$ getting $g_{B_{\gamma}}$. Endow $\partial T_{\gamma_{2}}\left(\gamma_{\delta_{1}}\right)$ with the induced orientation. Then $\left(B_{\gamma}, g_{B_{\gamma}}\right)$ is a bg, ne-bordism between $\left(M^{n}, g\right)$ and $\left(M^{n}, g\right) \cup$ 
$\left(\partial T_{\delta_{2}}\left(\gamma_{\delta_{1}}\right), g_{\partial T}\right)$. Theorem 4.28 yields

$$
\left(M^{n}, g\right) \cup\left(\partial T, g_{\partial T}\right) \underset{n e}{\sim}\left(M^{n}, g\right) \cup\left(\operatorname{chc}^{n}\left(\delta_{2}\right), g_{s t}\right) .
$$

THEOREM 4.31. $\Omega_{n}^{n c}(n e) \equiv \Omega_{n}^{n c}(b g, n e)$ is an abelian group with $-\left[M^{n}, g\right]=\left[\left(-M^{n}, g\right)\right]$ and $0=\left[\operatorname{chc}^{n}(r), g_{s t}\right]$.

Our next goal is to produce independent generators of $\Omega_{n}^{n c}(n e)$. As we shall see in the sequel, infinite connected sums of complex projective spaces (or their cartesian products) supply such elements. We prepare this by several assertions.

LEMma 4.32. Let $\left(M_{i}^{n}, g_{i}\right), i=1,2$, be open, oriented of bounded geometry and with a finite number of ends, each of them nonexpanding. Let further $\left(B^{n+1}, g_{B}\right)$ a ne-bordism between them and $K \subset B$ compact such that the ends of $B$ coincide with the components of $B \backslash K$. Let $C_{\varepsilon} \subset B \backslash K$ a component of $B \backslash K$ and $x_{0} \in C_{\varepsilon}$. Then there exists a constant $C_{1}>0$ such that the diameter of any metric sphere

$$
S_{\varrho}\left(x_{0}\right)=\left\{x \in C_{\varepsilon} \mid d_{B}\left(x, x_{0}\right)=\varrho\right\}
$$

is $\leq C_{1}$. Here the diameter is with respect to the induced length metric $d_{B}$ of $B$.

Proof. We start with the case that $M_{i} \backslash K$ contains exactly one end of $M_{i}$, respectively. Then there are geodesic rays $\gamma_{i} \subset M_{i} \backslash K \subset C_{\varepsilon} \subset B \backslash K$. For sufficiently large $\varrho, S_{\varrho}\left(x_{0}\right)$ intersects $\gamma_{i}$ in some points of a geodesic segment $I_{\varrho, c_{1}, i} \subset\left|\gamma_{i}\right|$ of length at most $c_{1, i}$, where

$$
d_{\gamma_{i}}(x, y)-c_{1, i} \leq d_{B}(x, y)
$$

for all $x, y \in\left|\gamma_{i}\right|$. Let $x_{i}=\sup I_{\varrho, c_{1}, i}\left(\left|\gamma_{i}\right|\right.$ is totally ordered $), i=1,2$. We would be done if we could show that $d_{B}\left(x, x_{1}\right)$ were uniformly bounded for all $x \in S_{\varrho}\left(x_{0}\right)$ and for all $\varrho>0$. Since $C_{\varepsilon} \subseteq U_{R}\left(\left|\gamma_{i}\right|\right)$ for some $R>0$, there exists for each $x \in S_{\varrho}\left(x_{0}\right)$ an $x_{i, \gamma_{i}}=x_{i, \gamma_{i}}(x) \in\left|\gamma_{i}\right|$ such that $x_{i, \gamma_{i}}(x) \in\left|\gamma_{i}\right|$ (which is equivalent to $x \in U_{R}\left(x_{i, \gamma_{i}}(x)\right)$ ). If $d_{B}\left(x_{i}, x_{i, \gamma_{i}}(x)\right)$ were uniformly bounded then we would be done:

$$
d\left(x, x_{i}\right) \leq d\left(x_{i}, x_{i, \gamma_{i}}(x)\right)+d\left(x_{i, \gamma_{i}}(x), x\right) \leq d\left(x_{i}, x_{i, \gamma_{i}}(x)\right)+R .
$$

Suppose $x_{0}=\gamma_{1}(0)$. If $x_{1, \gamma_{1}}(x)<x_{1}$ then we obtain

$$
\begin{aligned}
& d_{B}\left(x_{0}, x\right) \leq d_{B}\left(x_{1, \gamma_{1}}(x), x_{0}\right)+d_{B}\left(x_{1, \gamma_{1}}(x), x\right) \leq d_{\gamma_{1}}\left(x_{1, \gamma_{1}}(x), x_{0}\right)+R . \\
& \varrho \leq-d_{\gamma_{1}}\left(x_{1, \gamma_{1}}(x), x_{1}\right)+\varrho+R, \\
& d_{\gamma_{1}}\left(x_{1, \gamma_{1}}(x), x_{1}\right) \leq R, \quad d_{B}\left(x_{1, \gamma_{1}}(x), x_{1}\right) \leq R .
\end{aligned}
$$

In the case $x_{1, \gamma_{1}(x)}>x_{1}$ we obtain

$$
\begin{aligned}
& \varrho+d_{\gamma_{1}}\left(x_{1}, x_{1, \gamma_{1}}(x)\right)-c_{1,1} \leq d_{B}\left(x_{0}, x\right)+d_{B}\left(x, x_{1, \gamma_{1}}(x)\right) \leq \varrho+R, \\
& d_{B}\left(x_{1}, x_{1, \gamma_{1}}(x)\right) \leq d_{\gamma_{1}}\left(x_{1}, x_{1, \gamma_{1}}(x)\right) \leq R+c_{11} .
\end{aligned}
$$

If $x_{0} \notin\left|\gamma_{1}\right|$ then we have to add to the right hand sides in (4.36), (4.38) a constant. The same estimates hold if we replace in (4.34)-(4.38) $1 \rightarrow 2$. Assume now that $M_{i} \backslash K$ splits into a finite number of ends $e_{i, 1}, \ldots, e_{i, r_{i}}, i=1,2$. Then

$$
C_{\varepsilon}=B \backslash K \subset \bigcup_{1}^{r_{1}} U_{R}\left(\left|\gamma_{1, i}\right|\right), \quad C_{\varepsilon}=B \backslash K \subset \bigcup_{1}^{r_{2}} U_{R}\left(\left|\gamma_{2, j}\right|\right) \text {. }
$$


$S_{\varrho}\left(x_{0}\right)$ has uniformly bounded diameter if $S_{\varrho}\left(x_{0}\right) \cap U_{R}\left(\left|\gamma_{i, j}\right|\right)$ has uniformly bounded diameter. But the latter case we just solved above.

Now we recall the chopping theorem of Cheeger/Gromov (theorem 3.33 in section 3) which is a consequence of Abresch's habilitation.

THEOREM 4.33. Suppose $\left(M^{n}, g\right)$ open, complete with bounded sectional curvature $|K| \leq$ $C$. Given a closed set $X \subset M^{n}$ and $0<r \leq 1$, there is a submanifold, $U^{n}$, with smooth boundary, $\partial U^{n}$, such that for some constant $c(n, C)$

$$
\begin{aligned}
& X \subset U \subset T_{r}(X), \\
& \operatorname{vol}(\partial U) \leq c(n, C) \operatorname{vol}\left(T_{r}(X) \backslash X\right) r^{-1}, \\
& |I I(\partial U)| \leq c(n, C) r^{-1} .
\end{aligned}
$$

Moreover, $U$ can be chosen to be invariant under $\mathcal{I}(r, X)=$ group of isometries of $T_{r}(X)$ which fix $X$.

In our case, $X=X_{\varrho}=\overline{B_{\varrho}\left(x_{0}\right)} \subset B^{n+1}$. To apply 4.33, we form $\left(V^{n+1}, g_{V}\right)=$ $\left(B^{n+1} \cup B^{n+1}, g_{B} \cup g_{B}\right)$ which is well defined and smooth since we assumed the Riemannian collar $\left.g_{B}\right|_{\text {collar }}=g_{\partial B}+d t^{2}$. Now we set $X_{V}=X \cup X$ and apply 4.33 . Fix $0<r \leq 1$. Then we get $U_{V}, H_{\varrho, V, r}=\partial U_{V}$.

$$
\begin{aligned}
& X_{V} \subset U_{V} \subset T_{r}\left(X_{V}\right)\left(=\left\{x \in V \mid d_{V}\left(x, X_{V}\right) \leq r\right\}\right), \\
& \operatorname{vol}\left(H_{\varrho, V, r}\right)=\operatorname{vol}\left(\partial U_{V}\right) \leq c(n+1, C) \operatorname{vol}\left(T_{r}\left(X_{V}\right) \backslash X_{V}\right) r^{-1} \\
& \left|I I\left(\partial U_{V}\right)\right| \leq c(n+1, C) r^{-1}
\end{aligned}
$$

and $U_{V}$ is invariant under $\mathcal{I}\left(r, X_{V}\right)$.

The main idea of the proof consists in considering the distance function $F=d\left(\cdot, X_{V}\right)$ where for points $\in V \backslash X_{V}, d\left(\cdot, X_{V}\right)=d\left(\cdot, X_{\varrho}\right)=d\left(\cdot, S_{\varrho}\right)$. Then one applies Yomdin's theorem (cf. [Yomdin]) to $F$ in Abresch's smoothed out metric. All constructions are invariant under the metric involution and this involution remains an isometry also with respect to Abresch's smoothed out metric.

Restricting the obtained $U_{V}, \partial U_{V}$ to $B$, we obtain the desired result for $X=\overline{B_{\varrho}\left(x_{0}\right)} \subset$ $B$. Restricting for $\varrho$ large to $C_{\varepsilon}$ and using the construction of $U$ as preimage under the smoothed $F$, we obtain in $\bar{C}_{\varepsilon}$ a hypersurface $H=H_{\varrho}$ which decomposes $\bar{C}_{\varepsilon}$ into a compact and noncompact part $\bar{C}_{\varepsilon, c}$ and $\bar{C}_{\varepsilon, n c}$, respectively. Under our assumptions $(\partial B$ is totally geodesic) it is possible to arrange that $H^{n}$ intersects $\partial B$ transversally under an angle $>\delta$ and that there exists a constant $C_{1}$ independent of $\varrho$ such that

$$
\left|I I\left(\partial H_{\varrho}^{n}\right)\right| \leq C_{1} .
$$

We infer from (4.40), bounded curvature and lemma 4.32 that for fixed $0<r \leq 1$ there is a constant $C_{2}>0$ such that

$$
\operatorname{vol}\left(H_{\varrho}^{n}\right) \leq C_{2}
$$

for all $\varrho$. Moreover, $H_{\varrho}^{n}$ has bounded geometry (at least of order 0 ) according to (4.41) and to the bounded geometry of $B$.

Now we are able to present independent generators of $\Omega_{4 k}^{n c}(n e)$. Let $P^{2 k}(\mathbb{C})$ be the complex projective space with its standard orientation and with its Fubini-Study metric, 
fix two points $z_{1}, z_{2}$ and form by means of fixed spheres about $z_{1}, z_{2}$ the infinite connected sum

$$
M^{4 k}=\left(M^{4 k}, g\right)=\underset{1}{\#} P^{2 k}(\mathbb{C}),
$$

always with the same glueing metric. Then $\left(M^{4 k}, g\right)$ is oriented, has bounded geometry, one end which is nonexpanding.

THEOREM 4.34. $M^{4 k}=\#_{1}^{\infty} P^{2 k}(\mathbb{C})$ defines a non zero bordism class in $\Omega_{4 k}^{n c}(n e)$.

Proof. Suppose $\left[M^{4 k}\right]=0$. Then there exists a bordism $\left(B^{n+1}, g_{B}\right), \partial B=M^{4 k} \cup$ $-\operatorname{chc}^{4 k}(r),\left.g_{B}\right|_{U_{\delta}(\partial B)}=g_{\partial B}+d t^{2}, U_{R}\left(M^{4 k}\right) \supseteq B, U_{R}\left(\operatorname{chc}^{4 k}(r)\right) \supseteq B$ and $d_{B} \geq d_{M}-c$, $d_{B} \geq d_{\text {chc }}-c$. We choose $z_{0} \in P_{1}^{2 k}(\mathbb{C}), K=\emptyset$ and obtain for any $\varrho>0$ a compact hypersurface $H_{\varrho}^{4 k} \subset B=B \backslash \Phi=C_{\varepsilon}$ which decomposes $B$ into a compact and noncompact part $\bar{B}_{c}$ and $\bar{B}_{n c}$, respectively, and which satisfies (4.42), (4.43) and has bounded geometry at least of order 0 with constants independent of $\varrho$. Then $\partial \overline{B_{c}^{4 k+1}}=$ $\left(\partial \overline{B_{c}^{4 k+1}} \cap M^{4 k}\right) \cup H_{\varrho} \cup\left(\partial \overline{B_{c}^{4 k+1}} \cap \operatorname{chc}^{4 k}\right)$. Here $\sigma\left(\partial \overline{B_{c}^{4 k+1}} \cap \operatorname{chc}^{4 k}\right)=0 . \sigma\left(\partial \overline{B_{c}^{4 k+1}}\right)$ must be zero since it is 0 -bordant (if one wants, after smoothing out). Hence

$$
0=\sigma\left(\partial \overline{B_{c}^{4 k+1}} \cap M^{4 k}\right)-\sigma\left(H_{\varrho}^{4 k}\right) .
$$

But

$$
\sigma\left(H_{\varrho}^{4 k}\right)=\int_{H_{\varrho}^{4 k}} L+\eta\left(\partial H_{\varrho}^{4 k}\right)+\int_{\partial H_{\varrho}^{4 k}} \operatorname{expression}\left(I I\left(\partial H_{\varrho}^{4 k}\right)\right) .
$$

The first expression on the r.h.s. of (4.46) is bounded by a bound independent of $\varrho$ according to (4.43) and $\left(B_{0}\right)$ for $H_{\varrho}^{4 k}$. The same holds for the second expression according to

$$
\left|\eta\left(\partial H_{\varrho}^{4 k}\right)\right| \leq C_{3} \operatorname{vol}\left(\partial H_{\varrho}^{4 k}\right)
$$

and for the third expression according to $(4.42),(4.43)$. On the other hand, choosing $\varrho$ sufficiently large, $\sigma\left(\partial \overline{B^{4 k+1}} \cap M^{4 k}\right)$ can be made arbitrary large. This contradicts (4.45).

Looking at the proof of theorem 4.34, we immediately infer

THEOREM 4.35. Let $\left(M^{4 k}, g\right)$ be open, oriented, of bounded geometry and with a finite number of ends, each of them nonexpanding. If for any exhaustion $M_{1} \subset M_{2} \subset \cdots$ by compact submanifolds, $\cup M_{i}=M$, we have

$$
\lim _{i \rightarrow \infty} \sigma\left(M_{i}^{4 k}\right)=\infty
$$

then $\left[M^{4 k}, g\right] \neq 0$ in $\Omega_{4 k}^{n c}(n e)$.

Corollary 4.36. $\#_{1}^{\infty} P^{2 k}(\mathbb{C})$, or, more generally, $P^{2 i_{1}}(\mathbb{C}) \times \cdots \times P^{2 i_{r_{1}}} \# P^{2 j_{1}}(\mathbb{C}) \times \cdots \times$ $P^{2 j_{r_{2}}} \# \cdots, i_{1}+\cdots i_{r_{1}}=k, j_{1}+\cdots+j_{r_{2}}=k, \ldots$ are not torsion elements in $\Omega_{4 k}^{n c}(n e)$.

A special case of theorem 4.35 is given by manifolds $M^{4 k}$ of the type

$$
M^{4 k}=\underset{1}{\#} M_{i}^{4 k}
$$

$\operatorname{vol}\left(M_{i}^{4 k}\right) \leq C_{1},\left|K\left(g_{i}\right)\right| \leq C_{2}, r_{\text {inj }}\left(g_{i}\right) \geq C_{3}>0, \sigma\left(M_{i}^{4 k}\right) \geq 0$ for $i \geq i_{0}$ and $>0$ for infinitely many $i \geq i_{0}$. Then, in particular, $\mathcal{H}_{2 k, 2}\left(M^{4 k}\right)$ is infinite-dimensional and $\left[M^{4 k}, g\right] \neq 0$ in $\Omega_{4 k}^{n c}(n e)$, i.e. adding a finite number of closed manifolds with negative 
signature and an infinite number of closed manifolds with zero signature (such that the bg, ne-end struture remains preserved) does not transform a nonzero element into zero in $\Omega_{4 k}^{n c}(n e)$. A finer characterization of nonzero elements in $\Omega_{4 k}^{n c}(n e)$ will be presented elsewhere. Moreover there are very interesting specializations of the theory developed until now and generalizations, e.g. the restriction to manifolds with warped product structure at infinity or with prescribed volume growth of the ends etc. This will be the topic of another investigation.

5. The Novikov conjecture for open manifolds. As is very well known, the Novikov conjecture for closed manifolds stimulated many outstanding topologists to prove this and on this road deep results in $C^{*}$ algebraic topology, $C^{*} K$-theory and geometric group theory have been achieved. Hence, the Novikov conjecture has not only its own meaning but even more meaning as a stimulating question.

If $M^{n}$ is open and we consider the classifying diagram

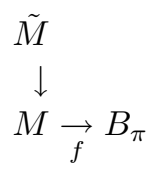

and $a \in H^{*}\left(B_{\pi}\right)$ then

$$
\left\langle L(M) \cdot f^{*} a,[M]\right\rangle
$$

will not be defined in general. For this reason, Gromov proposes to consider

$$
\sigma_{a}(M)=\left\langle L(M) \cdot f^{*} a,[M]\right\rangle
$$

for $a \in H_{c}^{*}\left(B_{\pi}\right)$. Then the NC for open manifolds would mean the "invariance of $\sigma_{a}(M)$ under proper homotopy equivalences". Probably much more appropriate would be an approach in the sense of our "open category", i.e.

1) everything is uniformly metrized, we have $(I),\left(B_{k}\right)$, uniform triangulations etc.,

2) maps are bounded and uniformly proper, in particular this holds for homotopy equivalences,

3) one works within functional algebraic topology.

Hence one should consider

$$
\left\langle L(M) \cdot f^{*} a,[M]\right\rangle \quad \text { with } L(M) \in L_{p}, f^{*} a \in L_{q} .
$$

Of particular meaning would be the cases

$$
L(M) \in{ }^{b} H^{*}(M) \quad \text { and } \quad a \in H^{*, 1}\left(B_{\pi}\right)
$$

or

$$
L(M) \in H^{*, 2}(M) \quad \text { and } \quad a \in H^{*, 2}\left(B_{\pi}\right),
$$

respectively. If we suppose $(M, g)$ satisfying $\left(B_{0}\right)$ then automatically $L(M) \in{ }^{b} H^{*}(M)$. $\left(B_{0}\right)$ does not restrict to topological type since any open manifold admits a metric $g$ satisfying even $\left(B_{\infty}\right)$ and $(I)$.

In the second case one should additionally assume

$$
\inf \sigma_{e}\left(\left.\Delta_{*}(M, g)\right|_{\left(\operatorname{ker} \Delta_{*}\right)}\right)>0,
$$


i.e. there is a spectral gap of $\Delta_{*}$ above zero. In this case $H^{*, 2}=\mathcal{H}^{*, 2}=L_{2}$-harmonic forms, $C^{*, 2}, C_{*, 2}$ are $L_{2}$-complexes and form an $L_{2}$-Poincaré complex. Every $L_{2}$-(co-)homology class can be represented by an $L_{2}$-harmonic (co-)cycle. Bordism of $L_{2}$-Poincaré complexes can be defined easily.

We proved in [8] that (5.3) is invariant under bounded uniformly proper homotopy equivalences. W.l.o.g., classifying maps can be assumed to be bounded and uniformly proper,

$$
M^{n} \rightarrow B_{\pi}=M^{n} \cup \text { cells. }
$$

We present now 3 versions of NC (for open manifolds).

First version. In the class of open oriented manifolds $\left(M^{n}, g\right), g \in{ }^{b, 2} \mathcal{M}^{2,2}\left(B_{0}, 2, f\right)$ with $\inf \sigma_{e}\left(\left.\Delta_{*}(g)\right|_{\left.\left(\operatorname{ker} \Delta_{*}\right)^{\perp}\right)>0 \text { is }}\right.$

$$
\left\langle L(M) f^{*} a,[M]\right\rangle, \quad a \in H^{*, 2}\left(B_{\pi}\right), \quad f \text { bounded and }
$$

uniformly proper classifying map, invariant under

bounded and uniformly proper homotopy equivalences.

Criticism. This version should hold only in very restricted cases. The starting point in the compact case is the equality

$$
\sigma\left(M^{4 k}\right)=\int L_{k}(M)
$$

where the l.h.s. is a priori a homotopy invariant and the r.h.s. is a certain characteristic number. The $L_{2}$-version of (5.4) is already wrong in simple open cases. Let $\left(M^{4 k}, g\right)$ be an open manifold with cylindrical ends, i.e. $\left(M^{4 k}, g\right)=\left(M^{\prime 4 k} \cup \partial M^{4 k} \times[0, \infty[, g)\right.$ with $\left.\left.g\right|_{\partial M^{\prime 4 k} \times[0, \infty[} \cong g\right|_{\partial M^{\prime}}+d t^{2}$. Then it is well known that

$$
\sigma\left(M^{4 k}\right)=\sigma_{L_{2}}\left(M^{4 k}\right)=\int L_{k}(M)-\eta\left(\partial M^{\prime 4 k}\right),
$$

i.e. already the starting point which guarantees the invariance of $L(M)$ in the simplest case is wrong. Hence the first version of NC for open manifolds makes sense only for those classes of manifolds for which

$$
\sigma_{L_{2}}\left(M^{n}\right)=\int L(M)
$$

in the case $n=4 k$.

Second version of NC, relative version. Fix $\left(M^{n}, g\right)$ and suppose that $M_{1}, M_{2} \in$ gen ${ }^{b}$ comp $_{L, \text { iso,rel }}(M, g)$,

$$
M_{1} \backslash K_{1} \cong M \backslash K, \quad M_{2} \backslash K_{2} \cong M \backslash K
$$

with a Riemannian collar at $\partial K_{1}, \partial K_{2}, \partial K$. Then we define

$$
\begin{aligned}
& \sigma\left(M_{i}, M\right):=\int_{K_{i}} L\left(M_{i}\right)-\int_{K} L(M), \\
& \sigma\left(M_{1}, M_{2}\right):=\sigma\left(M_{1}, M\right)-\sigma\left(M_{2}, M\right)=\int_{K_{1}} L\left(M_{1}\right)-\int_{K_{2}} L\left(M_{2}\right)=\sigma\left(K_{1} \cup K_{2}\right) \\
& \left.\quad=\int_{K_{1}} L\left(M_{1}\right)-\eta\left(\partial K_{1}\right)-\left(\int_{K_{2}} L\left(M_{2}\right)-\eta\left(\partial K_{2}\right)\right)\right)=\sigma\left(K_{1}\right)-\sigma\left(K_{2}\right) .
\end{aligned}
$$


The relative $\mathrm{NC}$ becomes

$$
\int_{K_{1}} L\left(M_{1}\right) f_{1}^{*} a=\int_{K_{2}} L\left(M_{2}\right) f_{2}^{*} a
$$

if there exist $\Phi_{12}: M_{1} \rightarrow M_{2}, \Phi_{21}: M_{2} \rightarrow M_{1}$, bounded, uniformly proper, $\Phi_{21} \Phi_{12} \sim$ id $M_{1}, \Phi_{12} \Phi_{21} \sim$ id $M_{2}$ bounded and u.p. and $\Phi_{21} \Phi_{12}=$ id outside $\tilde{K}_{1} \subset M_{1}, \Phi_{12} \Phi_{21}=$ id outside $\tilde{K}_{2} \subset M_{2}$ and $f_{i}: M_{i} \rightarrow B_{\pi}$ are bounded and u.p. classifying maps, $a \in H^{*}\left(B_{\pi}\right)$.

This relative version has the advantage that we require no conditions for $\left(M^{n}, g\right)$ and $\mathrm{NC}$ splits to NC for the generalized Lipschitz components.

Third version of NC. Consider $\left(M^{n}, g\right)$ open, oriented with $\left(B_{0}\right), r_{\text {inj }}>0$, embeddings $N^{4 k} \hookrightarrow M^{n} \times \mathbb{R}^{j}$ with trivial normal bundle and bounded second fundamental form such that $P D[N]=f^{*} a, a \in H^{n-4 k, 1}\left(B_{\pi}\right), f: M^{n} \rightarrow B_{\pi}$ bounded and uniformly proper classifying map and such that $\sigma_{L_{2}}\left(N^{4 k}\right)$ is defined (i.e. $\sim \mathcal{H}^{2 k, 2}(N)<\infty$ ).

Then the number $\sigma_{a}(M):=\sigma_{L_{2}}\left(N^{4 k}\right)$ is invariant under

bounded and uniformly proper homotopy invariants.

(NC03)

How to attack these conjectures will be the content of a forthcoming investigation.

\section{References}

[1] T. Aubin, Espaces de Sobolev sur les variétés Riemanniennes, Bull. Sc. Math. 100 (1976), 149-173.

[2] J. Cheeger and M. Gromov, Bounds on the von Neumann dimension of $L_{2}$-cohomology and the Gauß-Bonnet theorem for open manifolds, J. Diff. Geom. 21 (1985), 1-34.

[3] M. Cheeger and M. Gromov, On the characteristic numbers of complete manifolds of bounded curvature and finite volume, in: Differential Geometry and Complex Analysis, Springer, Berlin, 1985, 115-154.

[4] M. Cheeger and M. Gromov, Chopping Riemannian manifolds, in: Differential Geometry, Pitman Monogr. Surveys Pure Appl. Math. 52, 1991, 85-94.

[5] S. Cohn-Vossen, Kürzeste Wege und Totalkrümmung auf Flächen, Compositio Math. 2 (1935), 69-133.

[6] H. Donnelly, On the point spectrum for finite volume symmetric spaces of negative curvature, Comm. Part. Diff. Eq. 6 (1981), 963-992.

[7] J. Eichhorn, Global Analysis on Open Manifolds, Nova Science Publ., New York, 2006.

[8] J. Eichhorn, Invariants for proper metric spaces and open Riemannian manifolds, Math. Nachr. 253 (2003), 8-34.

[9] J. Eichhorn, Uniform structures of metric spaces and open manifolds, Results Math. 40 (2001), 144-191.

[10] J. Eichhorn, The signature for open manifolds, to appear.

[11] J.-H. Eschenburg, Comparison Theorems in Riemannian Geometry, Lect. Notes Series Dip. di Mat. Universita degli Studi die Trento, no. 3, 1994.

[12] M. Gaffney, A special Stokes' theorem for complete manifolds, Annals of Math. 60 (1954), 140-145.

[13] V. M. Goldstein, V. I. Kuzminov and I. A. Shvedov, The de Rham isomorphism of $L_{p^{-}}$ cohomology of noncompact Riemannian manifolds, Sibir. Mat. Zh. 29 (1988), 34-44. 
[14] A. Gray, Invariants of curvature operators of four-dimensional Riemannian manifolds, in: Proc. 13th Biennal Seminar Canad. Math. Congr., Montreal, 1972, 42-65.

[15] J. Heitschand C. Lazarov, A Lefschetz theorem on open manifolds, in: Contemp. Math. 105, Amer. Math. Soc., 1990, 33-45.

[16] A. Huber, On subharmonic functions and differential geometry in the large, Comm. Math. Helv. 32 (1957), 12-72.

[17] T. Januszkiewicz, Characteristic invariants of noncompact Riemannian manifolds, Topology 23 (1984), 289-301.

[18] D. Kucerovsky, Averaging operators on open manifolds, in: Contemp. Math. 148, Amer. Math. Soc., 1993, 151-153.

[19] J. Milnor and J. Stasheff, Characteristic Classes, Ann. Math. Studies 76, Princeton, 1974.

[20] M. S. Narasimhan and S. Ramanan, Existence of universal connections, Amer. J. Math. 83 (1961), 563-572.

[21] A. Polombo, Nombres caractéristiques d'une variété Riemannienne de dimension 4, J. Diff. Geom. 13 (1978), 145-162.

[22] S. Rosenberg, Gauß-Bonnet theorems for noncompact surfaces, Proc. AMS 86 (1982), 184-185.

[23] S. Rosenberg, On the Gauß-Bonnet theorem for complete manifolds, Trans. AMS 287 (1985), 745-753.

[24] L. Vanhecke, Geometry in Normal and Tubular Neighborhoods, Dep. Math., Kath. Univ. Leuven. 